Draft version OCtober 2, 2020

Typeset using $\mathrm{LAT}_{\mathrm{E}} \mathrm{X}$ twocolumn style in AASTeX63

\title{
A significant excess in major merger rate for AGNs with the highest Eddington ratios at $z<0.2$
}

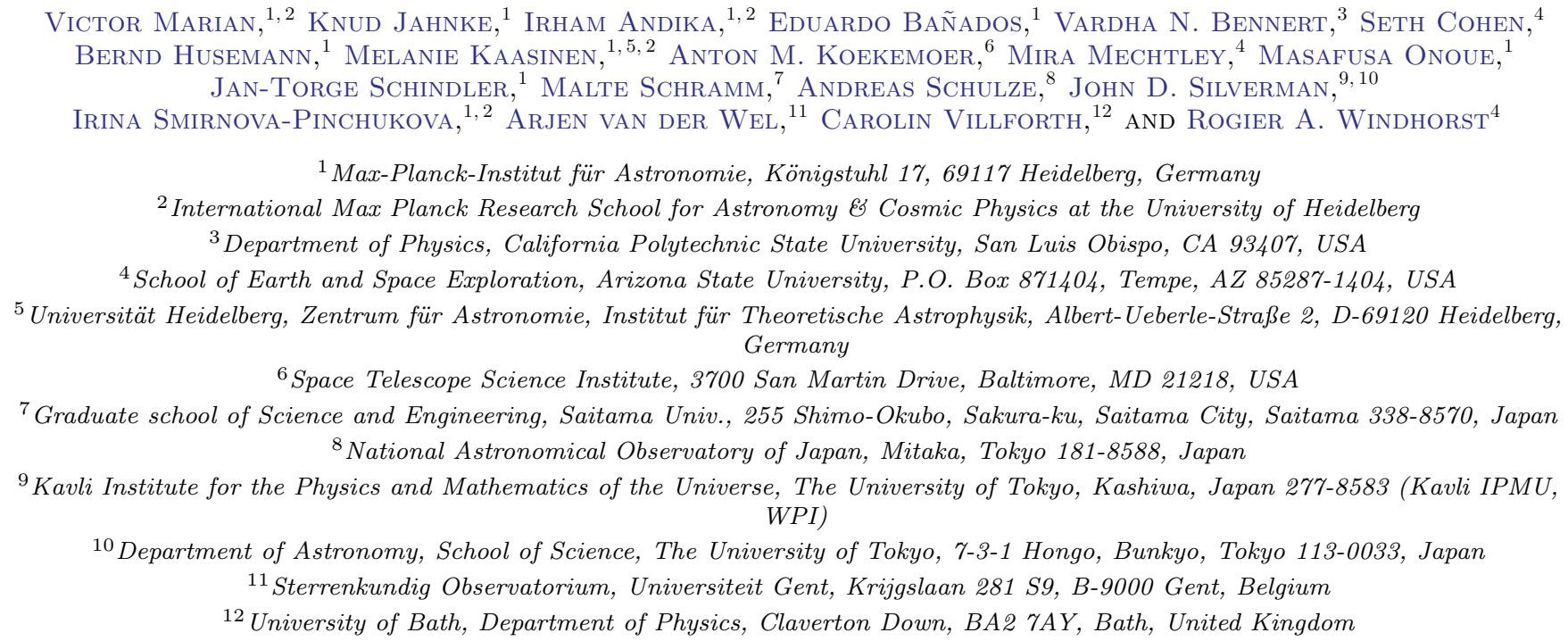

\begin{abstract}
Observational studies are increasingly finding evidence against major mergers being the dominant mechanism responsible for triggering AGN. After studying the connection between major mergers and AGN with the highest Eddington ratios at $z=2$, we here expand our analysis to $z<0.2$, exploring the same AGN parameter space. Using ESO VLT/FORS2 $B-, V-$ and color images, we examine the morphologies of 17 galaxies hosting AGNs with Eddington ratios $\lambda_{\text {edd }}>0.3$, and 25 mass- and redshift-matched control galaxies. To match the appearance of the two samples, we add synthetic point sources to the inactive comparison galaxies. The combined sample of AGN and inactive galaxies was independently ranked by 19 experts with respect to the degree of morphological distortion. We combine the resulting individual rankings into multiple overall rankings, from which we derive the respective major merger fractions of the two samples. With a best estimate of $f_{\mathrm{m} \text {,agn }}=0.41 \pm 0.12$ for the AGN host galaxies and $f_{\mathrm{m} \text {,ina }}=0.08 \pm 0.06$ for the inactive galaxies our results imply that our AGN host galaxies have a significantly higher merger rate, regardless of the observed wavelength or applied methodology. We conclude that although major mergers are an essential mechanism to trigger local high Eddington ratio AGNs at $z<0.2$, the origin of $\gtrsim 50 \%$ of this specific AGN subpopulation still remains unclear.
\end{abstract}

Keywords: galaxies: active — galaxies: evolution — galaxies: interactions — quasars: general

\section{INTRODUCTION}

An ever-growing number of empirical studies are finding that the properties of the black holes $(\mathrm{BH})$ at the center of galaxies are closely correlated with the properties of the host galaxy, i.e. BH mass, bulge velocity

Corresponding author: Victor Marian

marian@mpia.de dispersion and mass, stellar host mass, velocity dispersion or luminosity (e.g. Marconi \& Hunt 2003; Hring \& Rix 2004; Jahnke et al. 2009; Bennert et al. 2010, 2011; Beifiori et al. 2012; Graham \& Scott 2013; McConnell \& Ma 2013; Davis et al. 2018, 2019; de Nicola et al. 2019; Sahu et al. 2019; Shankar et al. 2019; Ding et al. 2020). These findings are complemented by state-of-theart cosmological hydrodynamical simulations (Habouzit et al. 2019; Li et al. 2019a; Terrazas et al. 2019) that attempt to capture the physics behind these relations. 
Combined with the widely-accepted assumption that every major galaxy hosts a supermassive $\mathrm{BH}$ in its center (Kormendy \& Ho 2013), this strongly indicates that hierarchical structure formation applies to black holes in the same way as it does to galaxies as a whole (Jahnke \& Macci 2011).

The potential feedback of the emitted radiation, winds, jets, or a combination thereof, when a $\mathrm{BH}$ becomes active, (i.e. starts accreting matter) may have a broad range of effects on the host galaxy, depending on the physical nature, geometry and/or size of those different outflow mechanisms (Silk \& Rees 1998; Harrison et al. 2018). These range from the total quenching to the enhancement of star formation due to various processes affecting the interstellar and circumgalactic medium (Husemann \& Harrison 2018; Weinberger et al. 2018; Davies et al. 2019; Nelson et al. 2019; Truong et al. 2019; Valentini et al. 2019; Oppenheimer et al. 2020), although the impact may also be negligible (Schulze et al. 2019; O'Leary et al. 2020). In addition, individual AGN feedback processes could even have an impact on larger scales by affecting satellite galaxies and the surrounding intracluster or intragroup medium (Blanton et al. 2010; Chowdhury et al. 2019; Dashyan et al. 2019; Li et al. 2019b; Martin-Navarro et al. 2019).

Considering this interplay between galaxies and their central $\mathrm{BH}$ in its active phase, it is imperative to understand the mechanisms responsible for triggering the period of significant black hole accretion. For decades it has been assumed that galaxies follow an evolutionary path that includes at least one merging event with another galaxy of a similar mass (i.e. a major merger). This gravitational encounter would strip part of the gas of its angular momentum, funneling it into the most central regions where the $\mathrm{BH}(\mathrm{s})$ reside (Barnes \& Hernquist 1992; Sanders \& Mirabel 1996). Such an incident would ultimately lead to the active galactic nucleus (AGN) phase, in which the coalescing galaxy hosts at least one active $\mathrm{BH}$ in the center. This theoretical scenario was comprehensively presented in the seminal work of Sanders et al. (1988), and further studied with numerous simulations (Springel et al. 2005; Hopkins et al. 2006a, 2008; Somerville et al. 2008; McAlpine et al. 2018, 2020; Weigel et al. 2018) and observations (e.g. Yue et al. 2019; Gao et al. 2020). These causal connections, between major mergers and the presence of an active BH, have been found especially for particular AGN populations at low redshift (Koss et al. 2010; Cotini et al. 2013; Sabater et al. 2013; Hong et al. 2015; Ellison et al. 2019), and high-luminosity AGNs at different cosmic epochs (Urrutia et al. 2008; Schawinski et al. 2012; Treister et al. 2012; Glikman et al. 2015; Fan et al.
2016; Donley et al. 2018; Goulding et al. 2018; UrbanoMayorgas et al. 2019).

In recent years, however, a number of studies have found that the fraction of major mergers amongst AGN hosts is $<50 \%$, implying that major mergers are not the dominant trigger of AGNs. For example, no predominant connection between major mergers and AGNs could be found for both the general population of X-raydetected and optically-observed AGNs at various redshifts (Gabor et al. 2009; Georgakakis et al. 2009; Cisternas et al. 2011). Likewise, studies that investigated luminosity-selected AGNs with low or moderate X-ray luminosities, with an upper limit of $L_{\mathrm{X}} \leq 10^{43} \mathrm{erg} \mathrm{s}^{-1}$ (Grogin et al. 2005; Allevato et al. 2011; Schawinski et al. 2011; Kocevski et al. 2012; Bhm et al. 2013) or high Xray luminosities with $L_{\mathrm{X}} \geq 10^{43} \mathrm{erg} \mathrm{s}^{-1}$ (Karouzos et al. 2014; Villforth et al. 2014, 2017) found no significant connection. Studies examining more specific samples of AGNs have obtained similar results: neither sources that possess the highest BH masses (Mechtley et al. 2016) nor heavily obscured AGNs (Schawinski et al. 2012; Zhao et al. 2019) appear to be triggered predominantly by major mergers. Even AGNs assumed to be in an early evolutionary stage (Villforth et al. 2019), or those exhibiting the highest Eddington ratios (Marian et al. 2019) show no signs of an enhanced merger fraction. Additional studies detected slight enhancements in the merger rate for AGNs at different luminosities and redshifts; however, the vast majority of AGNs were still not major merger induced (Silverman et al. 2011; Rosario et al. 2015; Hewlett et al. 2017). In contrast, recent work examining secularly powered outflows (Smethurst et al. 2019) and the dependence of local AGNs on environment (Man et al. 2019) suggest that secular processes are the dominant mechanisms to trigger AGN activity. These studies, in which AGNs with a variety of different redshifts, brightnesses and masses have been examined, have come to the unanimous conclusion that mergers should only be considered as one of several possible mechanisms for initiating black hole growth. Therefore, it is necessary to consider alternative processes and/or differences in the lifetime of merger features and AGNs.

Large-scale galactic bars (Cheung et al. 2015; Cisternas et al. 2015; Goulding et al. 2017) and a time delay between a major merger event and the onset of an AGN (Cisternas et al. 2011; Mechtley et al. 2016; Marian et al. 2019) appear to be an inadequate explanation for these contrary results regarding the relevance of large-scale mergers for triggering AGNs. Instead, Goulding et al. (2018) propose an intriguing alternative, which may ease this tension: although AGNs are indeed triggered by major mergers, their activity and therefore luminosity 
during the merging process depend on the merger stage and thus can vary heavily. At larger separations between the two galaxies the arising torques are not sufficient to provide enough gas to trigger an AGN phase or feed the black hole(s). However, at close passages the torques as well as the gas inflow increase, boosting the AGN activity, as long as the distance between the two galaxies is sufficiently small. Before coalescence, this would result in a periodic AGN variability, while the morphological features, like tidal tails, shells or asymmetries of this encounter would be continuously visible, explaining the lack of observed AGNs in merging systems.

In this study, we investigate the possibility that the AGNs with the highest Eddington ratios $\lambda_{\text {edd }}=L / L_{\text {edd }}$, i.e. the highest specific accretion rates at $z<0.2$ are predominantly triggered by major mergers. We also expand on the work presented in Marian et al. (2019), in which we studied comparable BHs at $z \sim 2$. Contrary to $z \sim 2$, which marks the peak of cosmic black hole activity (Boyle et al. 2000; Aird et al. 2015) and star formation rate (Madau \& Dickinson 2014), the comparable population of local AGN host galaxies at $z<0.2$ exhibit up to $\sim 10$ times lower black hole activity and star formation rates (Aird et al. 2015). Moreover, only a small fraction $(\lesssim 10 \%)$ of today's massive galaxies $\left(\log \left(M_{*} / \mathrm{M}_{\odot}\right)>10\right)$ may have undergone one or more major merger events since $z \sim 1$, with the majority of such galaxies being undisturbed for the past $\sim 7 \mathrm{Gyr}$ (Lpez-Sanjuan et al. 2009; Lotz et al. 2011; Xu et al. 2012). In addition, the mean black hole accretion rate (Delvecchio et al. 2015; Aird et al. 2019), as well as the cold gas fraction (e.g. Santini et al. 2014; Popping et al. 2015) of a galaxy are substantially lower at $z<0.2$ than at $z \sim 2$. Hence, we may expect different physical processes to be dominant at such a low redshift, which makes it necessary to also examine the role of major mergers with respect to triggering AGNs at such a cosmic time. Despite the expected small overall merger rates at low redshifts, especially for the particular population of AGNs showing the highest Eddington ratio, major mergers may still be the only viable option to deliver enough gas to the $\mathrm{BH}$ for it to reach such high specific accretion rates.

Like in almost all the aforementioned studies that reject major mergers as the dominant triggering mechanism of AGNs, we compare a specific sample of AGN host galaxies to a sample of inactive comparison galaxies, matched in redshift, stellar mass, observed wavelength, depth, and signal-to-noise ratio $(\mathrm{S} / \mathrm{N})$. We examine 17 galaxies hosting AGNs with $\lambda_{\text {edd }}>0.3$ at $z<0.2$ and 25 inactive control galaxies and compare the relative difference of the respective merger fractions in or- der to conclude whether major mergers play a dominant role. We derive the merger fractions by having experts visually classify and rank a joint blinded and randomized sample with respect to the appearance of distinct (major) merger features, such as tidal tails, shells, or asymmetries, which serve as proxies for an ongoing or recent past merger event. We then create a 'consensus ranking' and subsequently split the sample again into AGN hosts and inactive galaxies in order to determine the separate fraction of distorted sources as the basis for discussion.

All magnitudes are given in the $\mathrm{AB}$ system and we adopt a concordance cosmology, with $\Omega_{\Lambda}=0.7, \Omega_{0}=$ 0.3 and $h=0.7$. At our sample's median redshift of $z \sim 0.15 B$ - and $V$-approximately correspond to restframe $U$ - and $B$-band.

\section{DATA}

We base the sizes of our two samples on the goal to identify a potential predominant presence of major merger signatures in AGN host galaxies with respect to a matched sample of inactive galaxies. As a fiducial initial condition, we assume a merger fraction for our control sample of inactive sources of $f_{\mathrm{m} \text {,ina }}=0.15$ with the goal to be able to detect for an AGN host galaxy merger fraction of $f_{\mathrm{m} \text {,agn }} \geq 0.5$ a significance difference between these two fractions with $\sim 99 \%$ confidence. As the confidence of a detected difference in merger fractions can only increase for smaller values of $f_{\mathrm{m} \text {,ina }}$ and to ensure we achieve this desired level of confidence, we use this, when compared to literature results (e.g. Lotz et al. 2011; Man et al. 2016; Mundy et al. 2017), rather large value for $f_{\mathrm{m} \text {,ina }}$. We expect this fiducial fraction to be an upper limit of the real merger rate for inactive galaxies in our mass and redshift range.

Since the number of available AGNs with high Eddington ratios at $z<0.2$ is limited, we first create our sample of AGN host galaxies and then derive the number of inactive galaxies required to satisfy our conditions. With our final sample sizes we can then conclude whether or not AGN host galaxies show a significant enhancement in merger rates, indicating a causal dependence of our population of AGNs on major mergers.

\subsection{AGN Host Galaxies}

We construct our parent AGN sample by making use of the catalogs provided by the Hamburg/ESO survey (HES, Schulze \& Wisotzki 2010), the Palomar Green Survey (PG, Vestergaard \& Peterson 2006), and the SDSS DR7 (Shen et al. 2011). We constrain our selection of potential targets to sources with a redshift of $z<$ 0.2 . Since we require an estimate of the central $\mathrm{BH}$ mass 

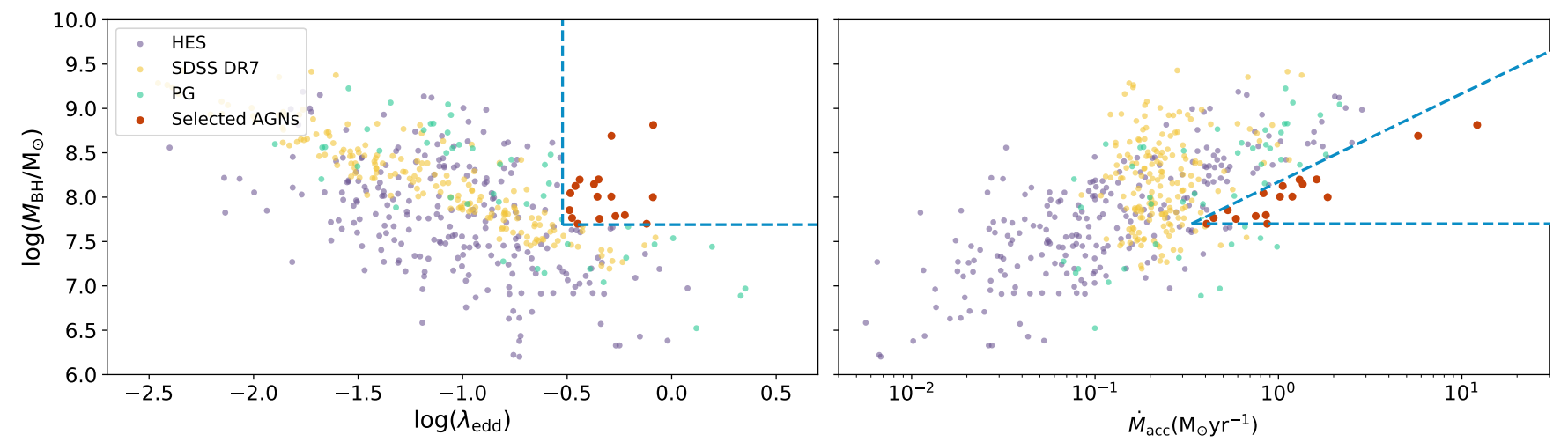

Figure 1. Left: Eddington ratio $\lambda_{\text {edd }}=L / L_{\text {edd }}$ vs. black hole mass for the parent sample of AGNs at redshift $z<0.2$. Overplotted are our selection limits in black hole mass and Eddington ratio (blue box) and our final selection of AGNs (red dots). Right: Black hole mass accretion rate vs. black hole mass for the same sample indicating that our final selection consists of AGNs possessing the highest specific accretion rates.

and are interested in the AGNs with the highest specific accretion rates, we only select unobscured broad-line AGNs with an Eddington ratio $\lambda_{\text {edd }}=L / L_{\text {edd }}>0.3$. To derive $\lambda_{\text {edd }}$, we use the $\mathrm{BH}$ mass determinations based on single epoch $\mathrm{H} \beta$ measurements and the bolometric luminosities, which, in turn, are based on the luminosities at $5100 \AA$ multiplied by a bolometric correction factor of $k_{\text {bol }}=9$ (Schulze \& Wisotzki 2010; Netzer 2019). Both the $\mathrm{BH}$ masses and luminosities at $5100 \AA$ are taken from the respective catalogs.

We apply a minimum $\mathrm{BH}$ mass threshold of $\log \left(M_{\mathrm{BH}} / \mathrm{M}_{\odot}\right)=7.7$, which results in a median $\mathrm{BH}$ mass for our AGN sample of $\log \left(M_{\mathrm{BH}} / \mathrm{M}_{\odot}\right) \sim 8.0$. Using the $\mathrm{M}_{\mathrm{BH}}-\mathrm{M}_{\text {bulge }}$ scaling relation of Kormendy \& Ho (2013) as a proxy to predict stellar host galaxy masses, the corresponding median stellar mass for our AGN host galaxies yields $\log \left(M_{*} / \mathrm{M}_{\odot}\right) \sim 11$. This mass selection results in feasible exposure times for our inactive galaxies, which are required to be of equal stellar mass, and enables us to compare the results presented in this work with the findings of (Marian et al. 2019), which are based on similar stellar host masses. Furthermore, we only select targets with a declination of $\operatorname{dec}<+15^{\circ}$ for better visibility with the VLT. All of these constraints yield a total number of 19 suitable AGN host galaxies, of which we observe 17 with VLT FORS2 in $V$ - and $B$-band (ESO programs 091.B-0672(A), 095.B-0773(A), and 098.A-0241(A), PI: Knud Jahnke). The median redshift of these 17 sources lies at $z=0.15$.

The left panel of Fig. 1 summarizes our selection process. The smaller colored points show the respective parent catalogs (with HES in violet, PG in green and SDSS in yellow) whereas the blue box shows the limits of our parameter space. Our final target selection is indicated by the red points. Since our AGNs show high
Eddington ratios $\left(\lambda_{\text {edd }}>0.1\right)$, we do not have to consider a potential trend of decreasing radiative efficiency $\eta$ with low accretion rates (Churazov et al. 2005; Weinberger et al. 2018; Nelson et al. 2019) and can calculate the $\mathrm{BH}$ mass accretion rates $\dot{M}_{\text {acc }}$ (Fig. 1, right panel) as

$$
\dot{M}_{\mathrm{acc}}=L / \eta c^{2}
$$

where we define $L$ as the derived bolometric luminosities and assume an efficiency parameter $\eta=0.1$. The right panel of Fig. 1 highlights that we target the AGNs with the highest specific accretion rates, i.e. those with the highest absolute mass accretion rates relative to their BH masses.

Each target has been observed for at least three long exposures, to detect large-scale distortion features down to $B$ and $V \sim 23.4 \mathrm{mag} / \operatorname{arcsec}^{2}$, and three short exposures, for an unsaturated image of the bright central region. The actual individual exposure times amount to $430 \mathrm{~s}$ and $14 \mathrm{~s}$ for $B$ and $150 \mathrm{~s}$ and $8 \mathrm{~s}$ for $V$, respectively. In Table 1, we summarize the properties of our AGN sample. We cite the corresponding catalog designations, redshifts, apparent $I$-band magnitudes, as well as the luminosities at $5100 \AA, L_{5100}$, and the bolometric luminosities, derived by applying a correction factor of 9 to $L_{5100}$ (Schulze \& Wisotzki 2010; Netzer 2019). In addition, we state the catalog values for the FWHM of the single-epoch measurements of the (broad) $H \beta$ line, the respective $\mathrm{BH}$ masses $M_{\mathrm{BH}}$, along with the calculated Eddington ratios $\lambda_{\text {edd }}$ and mass accretion rates $\dot{M}_{\text {acc }}$.

\subsection{Inactive comparison sample}

Given the size of the AGN sample and our assumptions for the merger fractions for our AGN and control sample $\left(f_{\mathrm{m} \text {,agn }} \geq 0.5\right.$ and $\left.f_{\mathrm{m} \text {,ina }}=0.15\right)$ we need to observe at least 25 inactive galaxies to meet our criterion 
Table 1. AGN sample properties

\begin{tabular}{lcccccccc}
\hline \hline AGN designation & $z$ & $m_{\mathrm{I}}$ & $L_{5100}$ & $L_{\mathrm{bol}}$ & FWHM & $M_{\mathrm{BH}}$ & $\lambda_{\text {edd }}$ & $\dot{M}_{\text {acc }}$ \\
& & $\mathrm{mag}$ & $\mathrm{erg} \mathrm{s}^{-1}$ & $\log \left(\mathrm{L}_{\odot}\right)$ & $\mathrm{H} \beta\left(\mathrm{km} \mathrm{s}^{-1}\right)$ & $\log \left(\mathrm{M}_{\odot}\right)$ & $\mathrm{M}_{\odot \mathrm{yr}^{-1}}$ \\
& $(2)$ & $(3)$ & $(4)$ & $(5)$ & $(6)$ & $(7)$ & $(8)$ & $(9)$ \\
\hline HE0119-2836 & 0.12 & 14.8 & 44.92 & 12.29 & 3363.00 & 8.2 & 0.36 & 1.3 \\
HE0132-0441 & 0.15 & 15.8 & 44.81 & 12.18 & 1719.00 & 8.0 & 0.44 & 1.0 \\
HE0157+0009 & 0.16 & 16.1 & 44.73 & 12.10 & 2369.00 & 7.8 & 0.60 & 0.9 \\
HE0444-3449 & 0.18 & 16.0 & 44.83 & 12.20 & 1714.00 & 8.1 & 0.35 & 1.1 \\
HE0558-5026 & 0.14 & 15.5 & 44.88 & 12.25 & 1583.40 & 8.0 & 0.51 & 1.2 \\
HE1201-2408 & 0.14 & 16.8 & 44.45 & 11.82 & 1820.86 & 7.8 & 0.33 & 0.4 \\
HE1226+0219 & 0.16 & 13.2 & 45.89 & 13.26 & 3835.03 & 8.8 & 0.82 & 12.1 \\
HE1228+0131 & 0.12 & 14.4 & 44.93 & 12.31 & 1866.19 & 8.1 & 0.43 & 1.4 \\
HE2011-6103 & 0.12 & 16.3 & 44.53 & 11.90 & 2862.51 & 7.9 & 0.32 & 0.5 \\
HE2152-0936 & 0.19 & 14.2 & 45.56 & 12.93 & 2183.42 & 8.7 & 0.52 & 5.8 \\
HE2258-5524 & 0.14 & 15.9 & 44.68 & 12.05 & 2419.42 & 7.8 & 0.54 & 0.8 \\
PG1001+054 & 0.16 & 16.3 & 44.74 & 12.11 & 1700.00 & 7.7 & 0.76 & 0.9 \\
PG1012+008 & 0.19 & 16.2 & 45.01 & 12.38 & 2615.00 & 8.2 & 0.45 & 1.6 \\
PG1211+143 & 0.09 & 14.3 & 45.07 & 12.44 & 1817.00 & 8.0 & 0.81 & 1.9 \\
SDSS-J032213.89+005513.4 & 0.18 & 16.1 & 44.72 & 12.09 & 2440.00 & 8.0 & 0.33 & 0.8 \\
SDSS-J105007.75+113228.6 & 0.13 & 15.7 & 44.57 & 11.94 & 1906.00 & 7.8 & 0.45 & 0.6 \\
SDSS-J124341.77+091707.1 & 0.19 & 16.8 & 44.41 & 11.78 & 1979.00 & 7.7 & 0.36 & 0.4 \\
\hline
\end{tabular}

Note-Properties of the AGNs in our sample: Columns 1-3, 6, and 7 are taken from the respective catalogs (Vestergaard \& Peterson 2006; Schulze \& Wisotzki 2010; Shen et al. 2011). The bolometric luminosities $L_{\mathrm{bol}}$ in column 5 are calculated by applying a bolometric correction factor of 9 to $L_{5100}$ (Schulze \& Wisotzki 2010; Netzer 2019). Column 6 presents the FWHM of the broad component of $\mathrm{H} \beta$. We calculate the Eddington ratios $\lambda_{e d d}$ and black hole mass accretion rates $\dot{M}_{\text {acc }}$ in column 8 and 9 by using the bolometric luminosities $L_{\mathrm{bol}}$, the respective $\mathrm{BH}$ masses $M_{\mathrm{BH}}$, and a radiative efficiency parameter of $\eta=0.1$.

of detecting a difference in those merger fractions with $\sim 99 \%$ confidence. The comparison galaxies are randomly chosen from a parent sample of $\sim 2900$ galaxies, which are part of the SDSS MPA/JHU catalog (Kauffmann et al. 2003; Brinchmann et al. 2004). We perform this initial selection by constraining the declination to dec $<10^{\circ}$ and the redshift to $z<0.2$, resulting in a median redshift of $z \sim 0.13$ for our control sample. Furthermore, we only choose sources that possess comparable stellar masses to our AGN host galaxies.

As described in Section 2.1, we adopt the $\mathrm{M}_{\mathrm{BH}}-$ $\mathrm{M}_{\text {bulge }}$ scaling relation of Kormendy \& Ho (2013) to derive the median stellar host mass for the AGN sample from the inferred $\mathrm{BH}$ masses. We restrict the inactive galaxies to a small range around the median derived stellar mass of the AGN host galaxies, $\log \left(M_{*} / \mathrm{M}_{\odot}\right)=$ $11 \pm 0.01$. Finally, we vet all potential sources against hard X-ray AGN signatures (Baumgartner et al. 2013), to remove any galaxies with a hidden, obscured AGN. In Table 2 we provide the coordinates, redshifts, $k$ - corrected and dereddened $I$-band magnitudes, and median stellar masses from the MPA-JHU catalog for our comparison galaxies.

With the exception of one source ${ }^{1}$, all of the 25 galaxies in our final sample were observed in the $B$ - and $V$-band with a comparable observational setup as for our AGN host galaxies. Each target has been observed with at least three individual, $470 \mathrm{~s}$ and $180 \mathrm{~s}$ long, exposures in $B$ and $V$, respectively. This selection and observational approach enables us to analyze two distinct samples of AGN host galaxies and inactive comparison galaxies, which are nonetheless matched in redshift, stellar (host) mass, depth, spatial resolution, filter band and $\mathrm{S} / \mathrm{N}$. Thus, we can directly compare potential relative differences in the merger fractions of both populations.

\footnotetext{
${ }^{1}$ Due to weather losses one target was only observed in $V$-band
} 
Table 2. Comparison galaxy sample properties

\begin{tabular}{lccccc}
\hline \hline Galaxy designation & $\alpha(\mathrm{J} 2000)$ & $\delta(\mathrm{J} 2000)$ & $z$ & $m_{\mathrm{I}}$ & $M_{*}$ \\
& deg & \multicolumn{1}{c}{$\mathrm{deg}$} & & $\mathrm{mag}$ & $\log \left(\mathrm{M}_{\odot}\right)$ \\
& $(2)$ & $(3)$ & $(4)$ & $(5)$ & $(6)$ \\
\hline Gal000232 & 0.164 & -0.013 & 0.08 & 16.9 & 11.0 \\
Gal003114 & 2.083 & -0.772 & 0.16 & 17.8 & 11.0 \\
Gal030481 & 19.605 & -9.962 & 0.11 & 17.8 & 11.0 \\
Gal050873 & 34.151 & -8.233 & 0.18 & 18.1 & 11.0 \\
Gal079769 & 50.365 & -6.309 & 0.16 & 18.0 & 11.0 \\
Gal095873 & 58.093 & -6.748 & 0.09 & 16.6 & 11.0 \\
Gal176221 & 132.158 & 7.598 & 0.13 & 17.9 & 11.0 \\
Gal185580 & 133.941 & 3.320 & 0.12 & 17.2 & 11.0 \\
Gal204260 & 137.351 & 9.810 & 0.16 & 18.0 & 11.0 \\
Gal210148 & 138.539 & 4.123 & 0.14 & 17.3 & 11.0 \\
Gal221730 & 140.921 & -0.891 & 0.14 & 18.5 & 11.0 \\
Gal270096 & 150.303 & -0.089 & 0.10 & 17.5 & 11.0 \\
Gal286443 & 153.515 & 7.057 & 0.10 & 17.2 & 11.0 \\
Gal347112 & 164.300 & 6.874 & 0.14 & 17.7 & 11.0 \\
Gal391560 & 171.878 & -2.142 & 0.10 & 17.1 & 11.0 \\
Gal419090 & 176.075 & -1.720 & 0.11 & 17.1 & 11.0 \\
Gal458007 & 181.927 & 1.421 & 0.11 & 17.5 & 11.0 \\
Gal498251 & 188.551 & -1.446 & 0.16 & 17.7 & 11.0 \\
Gal510223 & 190.692 & 0.540 & 0.08 & 17.0 & 11.0 \\
Gal510224 & 190.692 & 0.540 & 0.08 & 17.0 & 11.0 \\
Gal534882 & 195.327 & -0.937 & 0.19 & 18.2 & 11.0 \\
Gal557614 & 199.167 & 9.361 & 0.17 & 17.8 & 11.0 \\
Gal656010 & 215.724 & 8.849 & 0.14 & 17.3 & 11.0 \\
Gal676011 & 218.892 & 0.672 & 0.11 & 17.2 & 11.0 \\
Gal698144 & 222.606 & 6.647 & 0.16 & 18.0 & 11.0 \\
Gal782980 & 236.689 & -0.860 & 0.07 & 16.1 & 11.0 \\
\hline
\end{tabular}

Note-Our designations (column 1), coordinates (columns 2 and 3), redshifts (column 4$) k$-corrected and dereddened $I$-band magnitudes (column 5), and photometric median stellar masses for the inactive galaxies in our comparison sample taken from the MPA-JHU catalog (Kauffmann et al. 2003; Brinchmann et al. 2004).

\subsection{Data reduction and preparation}

We require a seeing of $1^{\prime \prime}$ or better to diagnose largescale merger signatures at a minimum required spatial resolution of $\sim 2.5 \mathrm{kpc}$ at our sample's median redshift. Hence, prior to reducing the raw images, we automatically determine the average seeing for each exposure by measuring the FWHM of 100 local peaks, using the Astropy package photutils (Bradley et al. 2019), and calculating the corresponding median FWHM of all sources. We visually check and re-measure every single exposure with a median FWHM $>1^{\prime \prime}$ and discard individual exposures with a median FWHM above this threshold. Out of a total of $\sim 450$ individual frames, we reject 22 from the subsequent reduction process and analysis. Despite the exclusion of these images, we end up with at least three individual exposures per band for every object.

To execute all the initial data reduction steps, i.e. the bias and flat-field correction, sky background subtractions, astrometry and aligning, and combination of individual exposures, we use the data processing pipeline 
THELI$^{2}$ (Erben et al. 2005; Schirmer 2013). The resulting pixel scale of 0.252 corresponds to $\sim 0.6 \mathrm{kpc}$ at our median redshift. We combine the respective $B$ - and $V$-band observations to create color images using MultiColorFits $^{3}$ (Cigan 2019).

To ensure that the samples are directly comparable, we mimic the appearance of the AGN host galaxies in the images of the inactive galaxies by adding a synthetic point source on top of the respective flux centers. To this end, we first detect the 15 brightest, unsaturated stars within the central image regions around each inactive galaxy with the help of the DAOStarFinder algorithm within the photutils package. For each galaxy, we then visually select and cut out one of the detected stars, and upscale the brightness correspondingly, such that they possess a central brightness comparable to HE2152-0936, our second brightest AGN source. In the course of this procedure we also downscale noise in the outer parts. Since an upscaling with a constant factor would lead to a noticeable discrepancy in flux between the galaxy and the edge of the artificially enhanced point source, we fit the original point sources with a 2D Gaussian and determine a circular region centered around the brightest pixel with a radius of $5 \sigma$. We divide this region into five bins and upscale the pixel values depending upon which bin they lie in. For the innermost region, i.e. within $1 \sigma$, we upscale with the total scaling factor, whereas for the outermost region, i.e. between 4 and $5 \sigma$ we apply a scaling factor lower by $5 \times 10^{-4}$. For the intermediate bins we choose a multiple of the scaling factor such that the distribution of the scaling factor with radius follows a Gaussian function. Using this approach we create point sources that resemble the central regions of our AGN host galaxies, but also blend in unrecognizably and smoothly into the respective galaxies. We add these point sources randomly at the centroid of each inactive galaxy, mimicking the appearance of our AGN host galaxies. Our point sources have a similar size to the upper limit of $\sim 1^{\prime \prime}$ set on the seeing, whereas the typical diameter of our sample galaxies, both AGN and inactive, is of the order of 5- $6^{\prime \prime}$. Thus, in contrast to our study of highly-accreting AGNs at $z \sim 2$ (Marian et al. 2019) there was no necessity to model and subtract point sources for the samples here.

An example of an AGN host galaxy and an inactive comparison galaxy are shown in Fig. 2. The left (a) and middle column (b) depict the $V$ - and $B$-band images, re-

\footnotetext{
${ }^{2}$ https://www.astro.uni-bonn.de/theli/gui/index.html https://github.com/schirmermischa/THELI

${ }^{3}$ https://multicolorfits.readthedocs.io
}

spectively, whereas the right column (c) shows the color images. To optimize the visibility of large-scale structures and possible merger signatures, while blending out the brightest inner regions, we chose different parameters for the color cuts and color map for the single band images as well as the color images. However, within one set, i.e. $V$-, $B$-band or color images, the parameters are constant. In addition, we adopted a Gaussian 2-pixel smoothing for the color images only. Due to the different visualization of the sources, we can test for any systematic differences in the subsequent distortion rankings or the resulting merger fractions (see Section 4).

\section{MORPHOLOGICAL ANALYSIS \& MERGER FRACTIONS}

We join both processed samples (for which the galaxies can no longer be visually separated as AGN or not) resulting in a final sample of 42 sources in $V$ - and 41 in $B$-band and color, respectively. To derive the merger fractions, 19 experts $^{4}$, proficient in working with imaging data of galaxies, perform a visual assessment of the targets, ranking them from most to least distorted with respect to the appearance of large scale distortion features. These features are indicative of ongoing or recent major merger events. Each set of $V$-, $B$-band and color images is ranked independently by each expert. We note that there are an increasing number of machine learning algorithms that can classify galaxies, based on their morphologies and possibly merger state (e.g. Bottrell et al. 2019; Cheng et al. 2019; Snyder et al. 2019). However, we rely on the human interpretation and judgment due to the manageable sample size and the extensive time and logistic requirement to teach an automatic classification routine with a matching 'external' training set. Since the sources in the joint sample are indistinguishable with respect to whether or not they are active, every expert's individual bias regarding the classification of a major/minor merger applies equally to AGN host galaxies and comparison galaxies. Thus, in our subsequent analysis any personal subjectivity in classification will have the same impact on either of the two subsamples. To further reduce any systematic bias, the dataset provided to each of the 19 ranking experts is randomized. As an additional task we request every classifier to choose a "cut-off" rank below which they deem all sources to be in a merging state, or, to at least show signs of a recent gravitational disturbance, like asymmetries,

\footnotetext{
4 The rankings were done by the coauthors Andika, Bañados, Bennert, Cohen, Husemann, Jahnke, Kaasinen, Koekemoer, Marian, Onoue, Schindler, Schramm, Schulze, Silverman, SmirnovaPinchukova, van der Wel, Villforth and Windhorst
} 

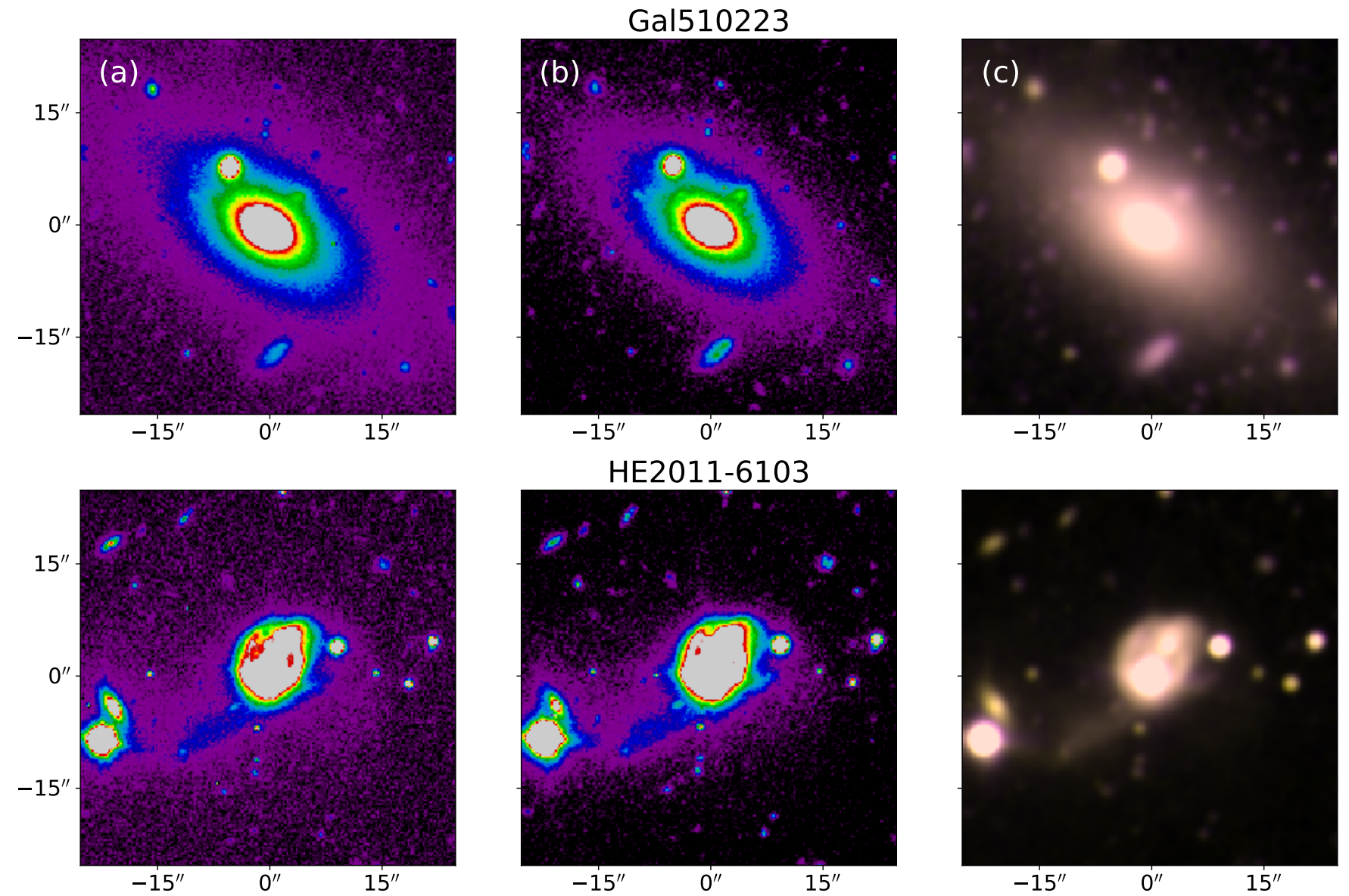

Figure 2. Two sources representative for our targets. On the top row we show one of the comparison galaxies and on the lower row one of our AGNs is displayed. From left to right we present a postage stamp in (a) V-band, (b) B-band and (c) color, respectively. Note: In order to enhance the visibility the images are not shown with the same cuts and color map parameters.

tidal tails or double nuclei. Every galaxy with a rank higher than the cut-off is interpreted to be completely free of major disturbances stemming from interactions. In our ensuing analysis we will use this property to determine the merger fractions of our two samples and also discuss the dependence of those fractions on different cut-off ranks (see Sect. 4).

We combine the 57 individual rankings (19 experts times three sets) into three consensus sequences for each respective set. We apply the same methods as in Marian et al. (2019) to combine the individual rankings and repeat this task for each set, i.e. separately for $B$-, $V$-band, and color images. For our first approach we calculate and weigh the average ranks of each galaxy, whereas for our second and third approach we use the Borda count (Emerson 2013) and Schulze algorithm (Schulze 2011, 2018), respectively. More information on the different methods and on how we implement them are provided in Appendix A. Ultimately, by applying all three methods to all three sets we obtain nine overall rankings.
We select various cut-off ranks and split the combined rankings back into AGN host and comparison galaxies. Subsequently, we derive the merger fractions for each chosen cut-off rank by counting how many active and inactive galaxies are above and below this threshold. The merger fraction is then simply defined as,

$$
f_{m}=\frac{a}{a+b}
$$

where $a$ represents the number of merging galaxies, whereas $b$ counts the sources that are undisturbed. However, since we only examine samples of limited size, we need to quantify the probability densities and uncertainties introduced by the shot noise for our resulting merger fractions. Based on those two parameters $a$ and $b$ we can quantify the probability densities for a continuous range of merger fractions in the feasible interval $[0,1]$ by using the beta distribution (see also Mechtley et al. 2016; Marian et al. 2019),

$$
f(x)=\frac{(a+b+1) !}{a ! b !} x^{a-1}(1-x)^{b-1} .
$$


Cut-off rank $=5$
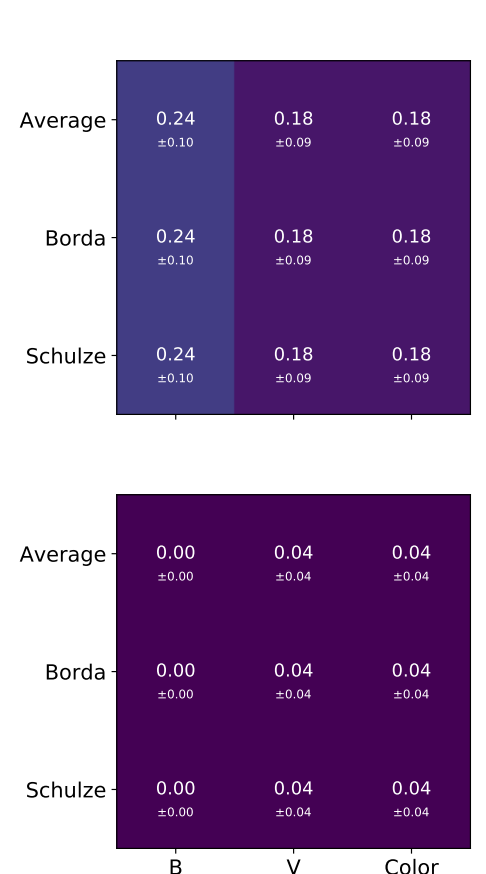

Cut-off rank $=10$

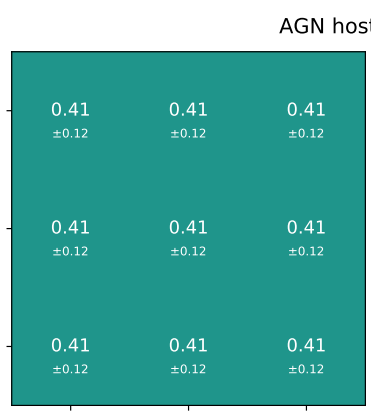

Comparison galaxies

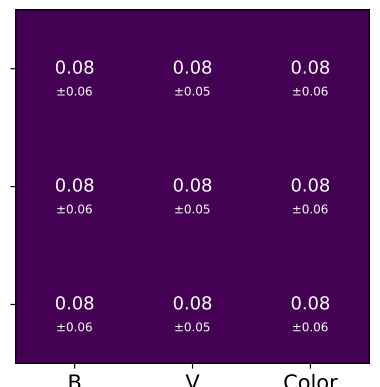

B

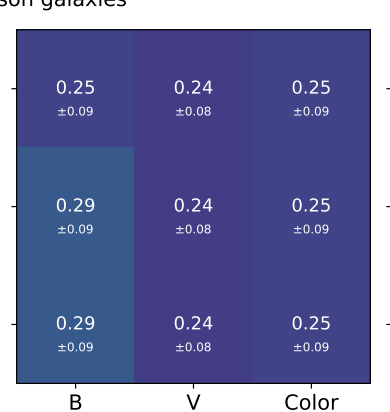

B
Cut-off rank $=15$

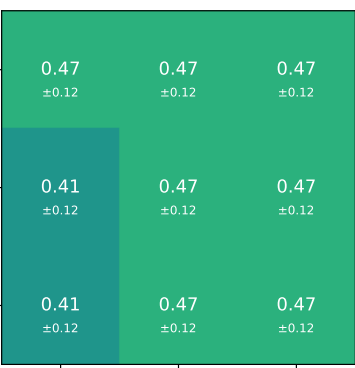

Cut-off rank $=20$
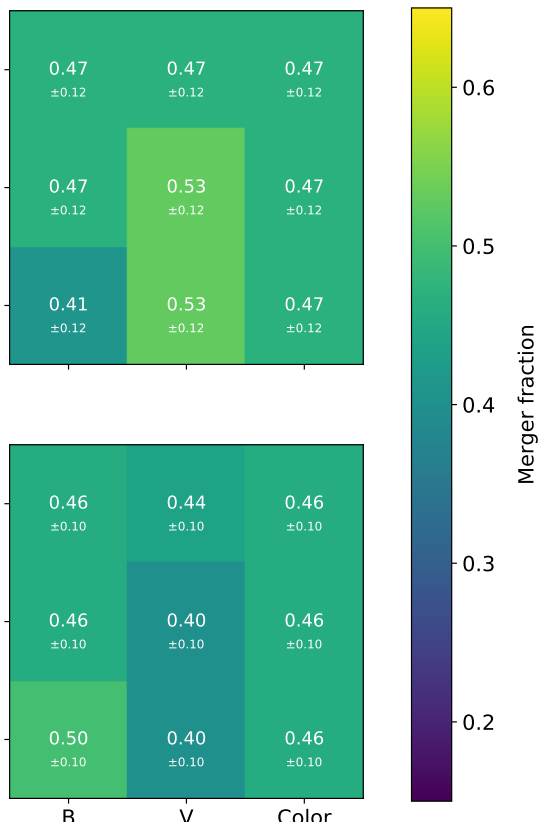

0.3

0.2

B V Color

Figure 3. The merger fractions for every set ( $B-, V$-band, and color images) and ranking combination method (Average, Borda, Schulze) for four distinct cut-off ranks. In the top row we show the corresponding fractions of disturbed AGN host galaxies, the bottom row depicts analogously the inactive comparison galaxies. The smaller numbers below the actual merger fraction values give the standard deviations (i.e. $1 \sigma$ ) of the corresponding beta distributions.

The respective standard deviations and means of the associated merger fraction probability distributions are then derived by,

$$
\sigma(x)=\sqrt{\frac{a b}{(a+b)^{2}(a+b+1)}},
$$

and Eq. 2, respectively.

In Figure 3, we present the corresponding means and standard deviations of the various probability distributions for every combination of method and set for four distinct cut-off ranks at 5, 10, 15, and 20. The merger fractions increase with cut-off rank, because a higher cut-off rank means that more galaxies are below this limit and are thereby considered to exhibit merger features. We find no evidence that the choice of combination method or the choice of $B-, V$-, or color image-set affect the resulting merger fractions. For all combinations the results for a given sample and cut-off rank are well within the errors of each other or even equal. However, it is also evident that for cut-off ranks $\lesssim 15$ the merger fractions for the AGN host galaxies (Fig. 3, upper row) are significantly larger then the fraction of disturbed inactive galaxies (Fig. 3, bottom row). This is not the case for larger cut-off ranks. We discuss the implications of the chosen cut-off ranks on our recovered merger fractions and the potential causal connection be- tween major mergers and the triggering of AGNs in the following section.

\subsection{Constraining the absolute merger fractions}

We have calculated the merger fractions for two samples of 17 AGN host galaxies and 25 inactive comparison galaxies. As mentioned in the preceding section, the final merger fractions depend on the choice of cut-off rank. In Appendix B we present the continuous evolution of merger fractions with cut-off rank for all combinations of set and method, while in this section we describe the two approaches we used to analyze and interpret our results. Firstly, we base the cut-off rank on our experts' opinions, and secondly, we construct this limit so that the resulting merger fraction of our inactive control sample is consistent with the merger rates presented in the literature. To obtain a valid first estimate, we calculated the means of the individual cut-off ranks chosen by each classifying expert for each set. The average cut-off ranks are $21 \pm 8,22 \pm 9$, and $18 \pm 8$ for the $B, V$, and color sets, respectively.

We suspect that the reason for such high cut-off ranks, which are almost bisecting our joint samples, lies in the visual determinations of our experts. Since our galaxies are well-resolved, any minor asymmetries (which do not need to stem from a recent major merger event, 
but can be of a minor merger or secular origin) can be easily identified. This leads our experts to put those particular sources into the 'merger bin', i.e. below the cut-off rank, increasing the percentage of galaxies classified as merging. With a corresponding cut-off rank = 20 , the merger fractions range between $f_{\mathrm{m}, \text { agn }}=0.41 \pm$ 0.12 and $f_{\mathrm{m} \text {,agn }}=0.53 \pm 0.12$ for the AGN sample and $f_{\mathrm{m} \text {,ina }}=0.40 \pm 0.10$ and $f_{\mathrm{m} \text {,ina }}=0.50 \pm 0.10$ for the inactive sample. Therefore, the fractions of disturbed sources in both samples are not significantly different, which would indicate a negligible contribution of mergers of any strength to the triggering of AGNs.

However, our primary goal is to determine the distinct impact of major mergers on the formation of AGNs, without considering the effects of minor gravitational encounters or other processes shaping the morphology of a galaxy. Thus, we have to correct our recovered merger fractions for the contamination by sources with minor asymmetries. Such a high merger rate of $\sim 40-$ $50 \%$, indicates that approximately half of the population shows signs of a recent or ongoing gravitational encounter of any strength. This significantly exceeds our initial assumption for inactive galaxies (see Sect. 2) and also the assessments by previous studies (Lotz et al. 2008a,b, 2011; Bridge et al. 2010; Xu et al. 2012; Casteels et al. 2014; Man et al. 2016; Ventou et al. 2017, 2019; Duncan et al. 2019; O'Leary et al. 2020). Based on these studies we adopt a major merger rate per galaxy of $R_{\mathrm{m}} \sim 0.05$ [Galaxy $\left.{ }^{-1} \mathrm{Gyr}^{-1}\right]$. This number represents the number of galaxies currently in a merger state, divided by the timescale of the visibility of merger signatures. In order to obtain an absolute merger fraction, representative of our comparison sample, we need to multiply this rate with the timescale $T_{\mathrm{m}}$ in which a major merger is observable. This property not only depends strongly on the mass ratio, individual masses, and gas fractions of the two progenitor galaxies, but also on the depth of the observations. Considering our targets' low redshifts and surface brightness limits we choose a comparatively conservative value of $t_{\mathrm{m}} \sim 1.5 \mathrm{Gyr}$, which results in a major merger fraction of $f_{\mathrm{m}} \sim 0.08$ for galaxies in our mass bin and at our sample's redshift.

Such a value for the merger fraction for our comparison galaxies corresponds to a cut-off rank $=10$. Coincidentally, at this cut-off rank the respective merger fractions are equal over all sets and methods for each of the two samples (Fig. 3) and yield $f_{\mathrm{m}, \text { agn }}=0.41 \pm$ 0.12 for the AGN host galaxies and $f_{\mathrm{m} \text {,ina }}=0.08 \pm 0.06$ for the comparison galaxies. This value of $f_{\mathrm{m} \text {,ina }}$ is not only in excellent agreement with the major merger rates found in the 3DHST survey by Man et al. (2016) for all five fields (AEGIS, COSMOS, GOODS-N, GOODS-

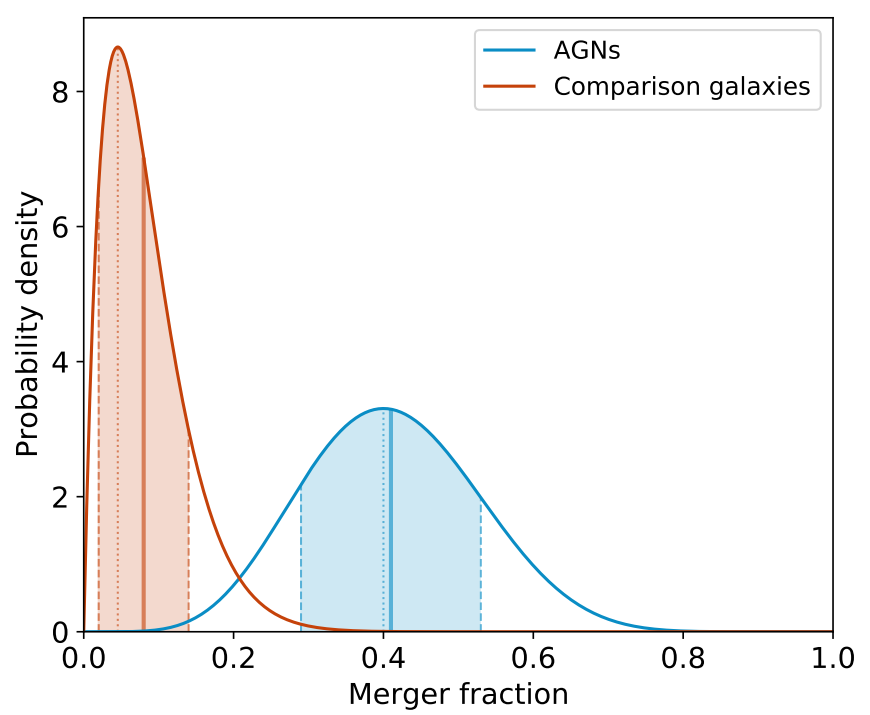

Figure 4. Probability distributions for the derived merger fractions of our $z<0.2$, high-accretion AGN host galaxies (blue) and inactive galaxies (red) at a cut-off rank $=10$. The solid and dotted lines show the means and modes of the respective merger fractions, while the dashed lines and shaded regions depict the central $68 \%$ confidence intervals. At this particular cut-off rank the respective merger fractions are identical, independent of method and set.

S, UDS) in CANDELS (Grogin et al. 2011; Koekemoer et al. 2011), but also for the major merger fractions recovered by MUSE deep observations (Ventou et al. 2017, 2019) as well as studies by Duncan et al. (2019) in CANDELS, and in GAMA by Mundy et al. (2017).

The two corresponding probability distributions are shown in Figure 4, with blue and red denoting the probability distributions for the AGN sample and the comparison sample, respectively. The shaded regions represent the $1 \sigma$ intervals and the solid and dotted lines depict the corresponding means and the modes. Due to the low number of merging comparison galaxies the associated probability distribution appears considerably skewed with the corresponding mean not coinciding with the peak position. Thus, we also report the merger fraction associated with the mode of the distribution, which yields $f_{\mathrm{m} \text {,ina }} \sim 0.04$ and is still well within the error of the mean.

\section{DISCUSSION}

\subsection{Robustness of results}

For a cut-off at rank 10, the resulting merger fractions translate to,

- 7/17 AGN host galaxies showing merger features, and, 
$-2 / 25$ inactive galaxies showing merger features.

The order and appearance of the sources in the various consensus rankings do not have to be congruent, e.g. the 7 as merger classified AGN host galaxies could vary in the different consensus rankings. However, we find that, despite a difference in order, the first eight positions of every combined ranking feature the same targets, with seven of them being the same AGN host galaxies. Out of these seven targets, five stem from the HES sample, while one each is listed initially in the SDSS and PG catalogs, respectively. Since we selected a total of 11 AGNs from the HES catalog and in each case three from the SDSS and PG catalogs, we conclude that the parent catalogs from which the AGN host galaxies are drawn from are not introducing any bias with respect to morphological classification. A repeated visual inspection also confirms that a distinction at exactly this cut-off rank into merging and non-disturbed systems reveals a noticeable separation into sources with clearly obvious large-scale merger features like tidal tails and shells and galaxies with explicitly less asymmetries.

Eventually, we created one singular overall ranking by re-applying the Schulze method on the nine consensus rankings (see Appendices $C$ and $D$ ). The same sources that occupy the first eight ranks in the nine initial consensus sequences, populate the highest positions in this final ranking as well. Therefore, we obtain an unchanged result for both merger fractions after again applying a cut-off at rank 10.

Considering the appearance of seven AGN host galaxies among the eight highest-ranked sources and the clear excess in merger fractions for the AGN host galaxies with respect to the inactive sample with a significant difference of $>2.5 \sigma$, we conclude that major mergers are an essential triggering mechanism for AGNs with the highest Eddington ratios at $z<0.2$. However, based on the mean of our recovered probability distribution for the AGN merger fraction we only find a $\sim 22 \%$ probability that the merger fraction is above the threshold of $f_{\mathrm{m} \text {,agn }}=0.5$. This means that although major mergers are indeed a non-negligible mechanism in triggering our specific population of AGNs, more than half of the BHs must be activated by different means, like secular processes or minor mergers. We discuss the role of the latter in triggering AGNs with the highest specific accretion rates at low redshifts in more detail in Sect. 4.5.

\subsection{Comparison to previous studies}

Our result, which shows an excess in AGN merger fraction compared to a matched control sample, stands in contrast to recent simulations (Steinborn et al. 2018; Ricarte et al. 2019) and several previous empirical stud- ies examining the potential causal connection between major mergers and the triggering of different populations of AGNs. Villforth et al. (2014) found no increase in merger signatures with luminosity and also reported consistent disturbance fractions between the AGNs and comparison galaxies for their sample of observed lowand moderate-luminosity $\operatorname{AGNs}\left(41 \lesssim L_{X}\left[\mathrm{erg} \mathrm{s}^{-1}\right] \lesssim\right.$ 44.5) at $0.5 \lesssim z \lesssim 0.8$. In contrast, Silverman et al. (2011) found an enhanced merger rate for AGNs of moderate X-ray luminosities in spectroscopic pairs at $z<1$. However, their rate of $17.8_{-7.4}^{+8.4} \%$ is still significantly lower than what we find here.

AGNs and host galaxies at comparable redshifts and luminosities as our sample were explored by Bhm et al. (2013) and Grogin et al. (2005). They assessed the neighboring counts, asymmetries, and various morphological indices (concentration, Gini coefficient and $M_{20}$ index) to characterize the respective host galaxies, but found no significant causality between major mergers and AGNs. Likewise, Allevato et al. (2011), Schawinski et al. (2011), and Rosario et al. (2015) detected no redshift evolution of morphological properties for similar AGNs up to $z \sim 2.5$ and Kocevski et al. (2012) found that only $16.7_{-3.5}^{+5.3} \%$ of comparable AGNs at $z \sim 2$ are highly disturbed. X-ray-selected and optically observed AGNs with higher luminosities $\left(43 \lesssim L_{X}\left[\mathrm{erg} \mathrm{s}^{-1}\right] \lesssim 46\right)$ at $0.5 \lesssim z \lesssim 2.2$ also appear to show no causal link to major mergers (Cisternas et al. 2011; Hewlett et al. 2017; Villforth et al. 2017). Instead they all reported consistent merger fractions of $\sim 15-20 \%$. Regarding more specific populations at $z \sim 2$ Schawinski et al. (2012) presented a major merger fraction between $4 \%$ and $11 \%$ for their analyzed sample of 28 dust-obscured AGNs, while Mechtley et al. (2016) has found consistent merger fractions for 19 galaxies hosting the most massive supermassive $\mathrm{BH}\left(M_{\mathrm{BH}}=10^{9}-10^{10} \mathrm{M}_{\odot}\right)$ and a sample of 84 matched inactive galaxies. Similarly, in our previous work (Marian et al. 2019) in which we examined 21 AGNs with the highest Eddington ratios $\left(\lambda_{\text {edd }}>0.7\right)$ at $z \sim 2$ and compared them to 92 matched inactive galaxies we found no dominant connection between major mergers and the occurrence of AGNs.

Similar to the results presented in this work other studies have found considerably enhanced merger rates for particular populations of AGNs. For their sample of hard X-ray detected, moderate luminous AGNs at $z<0.05$ Koss et al. (2010) reported an enhanced merger fraction of $18 \%$ when compared to a matched control sample, in which only $1 \%$ of the sources display merger features. However, they speculated that their AGNs may not be classified correctly via means of optical diagnostics due to superimposing features of ongoing star 
formation and optical extinction. In fact it appears that, independent of redshift, obscured and luminous AGNs are more likely to be connected to major merger events. Albeit, it should be noted that this is expected since by focusing on obscured sources a bias towards merging systems is most likely introduced as that obscuration may be due to dust within a merging (U)LIRG-like host. With this caveat in mind, Glikman et al. (2015), Fan et al. (2016), and Donley et al. (2018) detected merger fractions $>50 \%$ for such reddened or obscured AGNs sources at $z \sim 2, z \sim 3$, and $0<z<5$, respectively. Also at low redshifts $(z \lesssim 0.2)$ Koss et al. (2018) and Ellison et al. (2019) presented comparable results. In addition, in the latter study the authors described an increase of merger fraction with AGN luminosity, with the most luminous AGNs exhibiting the highest merger incidence. Corresponding findings have also been reported by Treister et al. (2012), Hong et al. (2015) and Goulding et al. (2018), who have analyzed luminous AGNs $\left(\log \left(L_{\text {bol }}\left[\mathrm{erg} \mathrm{s}^{-1}\right]\right)>45\right)$ at various redshifts. Especially with a merger fraction of $\sim 44 \%$ for luminous AGNs at $z<0.3$ the results published in Hong et al. (2015) are very consistent with the distortion rate we find for our sample of AGNs of comparable bolometric luminosity. Similar results are also reported by Gao et al. (2020) for their sample of AGNs at $0<z<0.6$, who detected a merger fraction of $\sim 40 \%$ and a general increase of distortion incidence with stellar mass. Finally, McAlpine et al. $(2018,2020)$ reported for the EAGLE simulation that major mergers - while of no great importance at high redshifts - play a significant role at low redshifts and present a consistent major merger fraction of $\sim 40 \%$ for BHs growing rapidly at $z \sim 0$.

\subsection{Physical interpretation and comparison to AGN counterparts at $z \sim 2$}

In light of our previous work at $z \sim 2$ (Marian et al. 2019), which also focuses especially on AGNs with the highest Eddington ratios, but yields an opposite result, we need to consider the different epochs of the studied AGNs. To make a comparison in absolute terms between the AGN major merger fractions, which we recover for the respective two samples at $z \sim 2$ and $z<0.2$, we have to factor in the impact of surface brightness dimming on detecting possible faint morphological distortion features. With a drop in surface brightness of $\sim 5 \mathrm{mag} / \operatorname{arcsec}^{2}$ at $\mathrm{z} \sim 2$, we miss at this redshift most definitely merger features we otherwise would see at $z \sim 0.2$. This effect can be enhanced by the fact that galaxies at $z \sim 2$ are on average more compact than at $z \sim 0$ (e.g. van der Wel et al. 2014). If the triggering of an AGN follows immediately after a starburst caused by a galaxy merger, the resulting potential extensive amount of dust can obscure the starburst at $z \sim 2$ more easily than at $z \sim 0.2$. In the latter case the starburst may happen as much in the galaxy's outer spiral arms and tidal streams, whereas the starburst in a galaxy at $z \sim 2$ is much more confined to the central region due to its more compact nature. Hence, in addition to the difference in surface brightness dimming between $z \sim 2$ and $z \sim 0.2$, a more complex situation is possible where the visibility of an AGN host galaxy at $z \sim 2$ is not only reduced by surface brightness dimming, but also obscuring dust. Thus, the AGN merger fraction at $z \sim 2$ could be significantly underestimated with $f_{\mathrm{m} \text {,agn }}$ $=0.24 \pm 0.09$ for the AGN sample at $z \sim 2$ and $f_{\mathrm{m} \text {,agn }}$ $=0.41 \pm 0.12$ for the AGNs presented in this study (see Sect. 3.1). Therefore, this effect could explain the discrepancy in the derived AGN major merger rates and would lead us to the conclusion that a substantial part of AGNs with the highest Eddington ratios at $z \sim 2$ is actually triggered by major mergers as well. However, in Marian et al. (2019) as well as in this study we draw our main conclusions by comparing the respective AGN samples to two matched control samples of inactive galaxies at both redshifts and determining primarily the relative differences between the respective merger fractions. The corresponding merger fractions for the inactive galaxies are $f_{\mathrm{m} \text {,ina }}=0.19 \pm 0.04$ and $f_{\mathrm{m} \text {,ina }}=$ $0.08 \pm 0.06$ for the sources at $z \sim 2$ and $z \sim 0.2$ (see Sect. 3.1), respectively.

We assume now that the actual merging process is independent of the presence of a potential future AGN and consider the mechanisms causing the detectable morphological features to be identical between the respective AGN host galaxies and their corresponding inactive counterparts. As a result the merger fractions at $z \sim 2$ are affected equally by surface brightness dimming and we actually do not have to consider this effect. Similarly, a merger-driven starburst creating an abundant amount of obscuring dust can happen equally in both an inactive galaxy or a system that will host an AGN triggered by this merger event. Hence, dust would only impact the findings described in Marian et al. (2019) if the dust were to obscure the actual AGNs, which would lead to a misclassification of those particular sources as inactive galaxies. In this earlier study, however, we investigated the importance of hidden and intermittent AGNs at $z \sim 2$, which would implicitly include such sources, but found no significant effect on the resulting merger rates. In addition, just as in this work, we deliberately have only selected type-1 AGNs, minimizing the proba- 
bility of dust obscured sources influencing the reported result. We expect the number of such sources with a dust content low enough to be not classified as type-2 AGNs, but sufficiently high to actually hide a potential AGN or morphological merger features to be relatively low. Therefore, similar to the surface brightness dimming, we can neglect the effect of obscuring dust when considering the relative difference in merger fractions at $z \sim 2$. A rigorous analysis would require a larger sample and data at longer wavelengths, as the James Webb Space Telescope (JWST) will be able to provide at $\mathrm{z} \sim 2$, enabling spatial modeling of dust in more detail.

Since we only compare the relative differences in merger fractions at both redshifts with no significant distinction of merger fractions at $z \sim 2$, but a clear excess of the AGN merger fraction when compared to inactive galaxies at $z<0.2$ we still conclude that major mergers play an essential role for AGNs with high Eddington ratios at low redshift. In addition, the major merger fractions for both samples of inactive galaxies are consistent with previous findings of major merger rates for galaxies at comparable redshifts and masses Man et al. (2016); Snyder et al. (2019); Steinborn et al. (2018); Ventou et al. (2017, 2019). This agreement corroborates the findings presented in Marian et al. (2019) and indicates that surface brightness dimming or dust is actually not impacting the merger fractions at $z \sim 2$ considerably. Hence, we have to consider an alternative explanation for this excess of merger fraction in our subpopulation of AGNs at low redshift.

Besides the mean $\mathrm{BH}$ accretion rate/bolometric luminosity and Eddington ratio of an AGN (Schulze et al. 2015 ), especially the cold gas fraction of a galaxy at $z<0.2$ is considerably lower than for a counterpart at $z \sim 2$ (e.g. Santini et al. 2014; Popping et al. 2015). Hence, with the AGNs in both redshift samples having comparable Eddington ratios, but the sources at lower redshifts a significant smaller intrinsic gas reservoir it is reasonable to assume that while at $z \sim 2$ a sufficient amount of gas is still left to fuel the central supermassive $\mathrm{BH}$ via other mechanisms than major mergers, at $z<0.2$ this process is essential to trigger AGNs with the highest specific accretion rates. This scenario is completely consistent with the results of the EAGLE simulations, which sees major mergers in a negligible role for triggering AGNs at high redshifts, but shows that such galaxy encounters play a substantial role at low redshifts, yielding comparable major merger fractions (McAlpine et al. 2018, 2020). However, it should be noted that despite the excess in major merger fraction for our AGN host galaxies, $\gtrsim 50 \%$ of our sample appear not to be not triggered by such an event, requiring an alternative explanation for the existence of such AGNs.

\subsection{AGN merger fraction and luminosity}

Although our AGN sources can be considered luminous for sources at $z<0.2$, we emphasize that we have not selected our AGNs on absolute luminosity (see Sect. 2.1 for our sample selection). Rather, we have chosen the AGNs with the highest Eddington ratios, i.e. the sources with the highest accretion rates and luminosities relative to their BH masses. Except for the two AGNs - HE1226+0219 and HE2152-0936, which possess bolometric luminosities of $\log \left(L_{\mathrm{bol}}\left[\mathrm{erg} \mathrm{s}^{-1}\right]\right)>46.5$ - all our remaining sample AGNs have luminosities of $45.3 \lesssim$ $\log \left(L_{\mathrm{bol}}\left[\mathrm{erg} \mathrm{s}^{-1}\right]\right) \lesssim 46$, but feature the smallest $\mathrm{BH}$ masses in that luminosity bin $\left(7.7<\log \left(M_{\mathrm{BH}} / \mathrm{M}_{\odot}\right)<\right.$ 8.2). In fact, $\sim 10$ more luminous AGNs in our three initial parent catalogs would have been selectable. Unlike other studies, which detect an enhanced merger rate for luminous AGNs we see no trend of the strength of the merger features - i.e. rank - with either $\mathrm{BH}$ mass or $\mathrm{BH}$ mass accretion rate/luminosity within our AGN sample (Fig. 5). In fact HE1226+0219 and HE21520936, both with distinctly higher absolute mass accretion rates with respect to our other sample AGNs, only occupy the ranks $\sim 30$ and $\sim 25$ in all the consensus rankings and show clearly no significant merger features. However, due to our selection of AGNs being based on a combination of $\mathrm{BH}$ mass and Eddington ratio, we note that apart from the two aforementioned most luminous AGNs our sources sample a relatively narrow luminosity range. Still, because of the lack of an obvious correlation of merger fraction with AGN luminosity, our results require an alternative explanation - especially considering that the existence of such a trend is still inconclusive. Despite some studies have found evidence of such a link between merger rate and luminosity (Treister et al. 2012; Fan et al. 2016; Goulding et al. 2018) others did not (Villforth et al. 2014, 2017; Hewlett et al. 2017).

\subsection{The (un)importance of minor mergers}

In Sect. 3.1 we argue that the initial high merger fraction of our sample of control galaxies, is the result of our experts including galaxies in the merger category, which show features that are only the consequence of minor merger events. Lotz et al. (2011) state that the minor merger rate is $\sim 3$ times the major merger rate (with a minor merger being in a mass ratio range of $\left.1: 4<M_{\text {sat }} / M_{\text {primary }} \lesssim 1: 10\right)$. Considering our major merger fraction for those galaxies to be correct we end up with a total merger fraction of $f_{\mathrm{m} \text {,ina }}=0.33 \pm 0.09$ for our inactive galaxies. This would correspond to a 

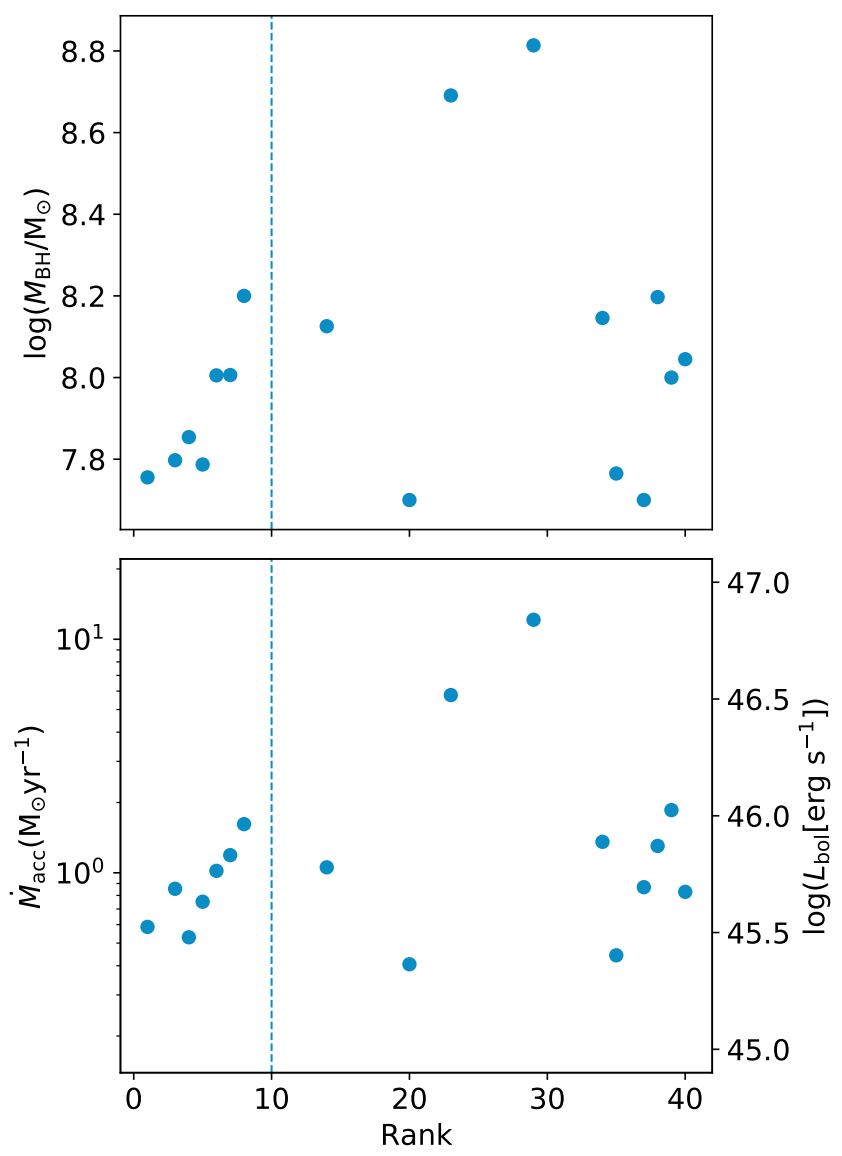

Figure 5. Overall consensus rank vs. black hole mass (top) and black hole mass accretion rate and bolometric luminosity (bottom) for our sample of AGNs. The vertical dashed line visualizes a cut-off at rank 10, which was used in our discussion.

cut-off at a rank of 17 and in turn in a total merger fraction of $f_{\mathrm{m}, \text { agn }}=0.47 \pm 0.12$ for our AGN host galaxies. Obviously the difference between these two distortion rates is significantly decreased and indeed for our singular overall ranking we find 8 inactive galaxies and 8 AGNs below our cut at rank 17. However, while all experts can easily agree on the most distorted galaxies, it should be noted that sources with such small asymmetries are more difficult to classify. Hence, the rank of a particular galaxy with such features may differ strongly in the individual expert's rankings, which in turn could also influence to some extent the resulting overall rank and hence also the actual number of sources being considered merging in our final ranking. Nevertheless, we do not expect this scatter to be substantial. With only one AGN host galaxy, but eight inactive galaxies added into the merger category, it appears that only a small fraction of AGNs are seen to be in this interval. Hence, we are confident that the number of AGN host galax- ies showing weak distortion features is still significantly less than compared to our comparison galaxies. This points to the conclusion that minor merging is comparably unimportant and most of the rest of AGNs require a different triggering mechanism.

\subsection{Considering AGN and merger timescales}

With major mergers only triggering at most $\sim 50 \%$ of our AGNs and minor mergers playing a subdominant role the question still remains which process(es) are responsible for triggering high Eddington rate AGNs at $z<0.2$. With that question in mind and a diminishing number of alternative mechanisms we consider a possible impact of the different timescales. Previous studies, which have found no enhancement in distortion fractions between AGNs and a matched sample of control galaxies, analyzed a potential disparity in AGN and merger lifetimes to be an explanation for their results (Cisternas et al. 2011; Mechtley et al. 2016; Marian et al. 2019). The unanimous conclusion is that the difference in life cycles is not sufficient to explain the lack of excess in merger rates, since the timescale of merger features being observable is much longer than the lifetime of the respective AGNs.

We consider a scenario in which some of the galaxies that host no visible AGN and feature only minor distortions are actually the result of a major merger event which also lead to a past phase of active black hole growth. However, since the lifetime of AGNs can be significantly shorter when compared to that of major merger features, the only detectable remains of such a gravitational encounter would be in the form of minor asymmetries. This implies that if we utilize the total merger fractions we derived in the previous subsection, a part of the $33 \pm 9 \%$ inactive galaxies that show distortions of various strength have actually hosted a major merger triggered AGN in the past. As a result the AGN major merger fraction with $f_{\mathrm{m} \text {,agn }}=0.41 \pm 0.12$ would increase, indicating that major mergers are not only an essential, but indeed the dominant mechanism to trigger high Eddington rate AGNs at $z<0.2$.

Following the scenario outlined by Goulding et al. (2018) we also assess the number of AGNs in an ongoing merger after first passage that are currently not visible due to an insufficient gas inflow. Those particular black holes will eventually become active again when the distance between the two galaxies decreases again resulting in growing torques and hence gas inflow. As in Marian et al. (2019), we refer to such AGNs in the following as intermittent AGN. We cannot distinguish between such AGNs or past AGNs that will not be ignited again. However, since we are only interested in 
the eventual increase of the AGN merger fraction, the origin of this increase is irrelevant.

We try to constrain the fraction of distorted inactive galaxies, which hosted an AGN in the recent past or currently an intermittent AGN, $f_{\mathrm{m} \text {,ina \& agn }}$ by adopting the formula presented in Marian et al. (2019):

$$
f_{\mathrm{m}, \text { ina } \& \text { agn }}=f_{\mathrm{agn}} \times f_{\mathrm{m}, \text { agn }} \times \frac{t_{\mathrm{m}}}{t_{\mathrm{agn}}} .
$$

Here, $f_{\text {agn }}$ and $t_{\text {agn }}$ represent the fraction and lifetime of AGNs with an Eddington ratio > 30\% with respect to the total galaxy population at our redshift and mass bin. The timescale in which the merger features are observable is given by $t_{\mathrm{m}}$, while $f_{\mathrm{m} \text {,agn }}$ describes the total merger fraction of our specific AGN population. We derive $f_{\text {agn }}$ by utilizing the number densities provided by stellar mass and quasar bolometric luminosity functions at $z \sim 0$ and our stellar mass range and average bolometric AGN luminosity. Using the respective median $I$-band magnitudes this yields $\log \Phi \sim-2.9 \mathrm{Mpc}^{-3} \mathrm{mag}^{-1}$ for the total galaxy population (Hirschmann et al. 2014; Henriques et al. 2015; Furlong et al. 2015; Lacey et al. 2016; Pillepich et al. 2018) and $\log \Phi \sim-5.8 \mathrm{Mpc}^{-3} \mathrm{mag}^{-1}$ for our particular population of AGNs (Hopkins et al. 2007; Fanidakis et al. 2012; Hirschmann et al. 2014; Sijacki et al. 2015), resulting in $f_{\text {agn }} \sim 1.3 \times 10^{-3}$, which is in excellent agreement with the value for the active fraction reported by Schulze \& Wisotzki (2010) for BHs at a redshift $z<0.3$ and a mass of $\log \left(M_{\mathrm{BH}} / \mathrm{M}_{\odot}\right) \sim 8$. For $f_{\mathrm{m} \text {,agn }}$ we use our reported value of $f_{\mathrm{m} \text {,agn }}=0.47 \pm 0.12$, but also repeat our calculations for $f_{\mathrm{m} \text {,agn }}=0.30$ and 0.70 . Besides our initial estimate of $t_{\mathrm{m}}=1.5 \times 10^{9} \mathrm{yr}$ (see Sect. 3.1), in addition, we use $t_{\mathrm{m}}=10^{9} \mathrm{yr}$ for comparison. Finally, in accordance to previous studies we constrain our AGN lifetime $t_{\mathrm{agn}}$ to a range between $10^{6}$ and $10^{8} \mathrm{yr}$ (Martini 2004; Hopkins et al. 2005; Shen et al. 2007; Hopkins \& Hernquist 2009; Conroy \& White 2013; Cen \& Safarzadeh 2015). Since we can not distinguish between inactive merging galaxies that already went through their AGN phase, are yet to host an AGN or are currently hosting an intermittent AGN, it is not necessary for us to consider any time lag (Hopkins et al. 2006b; Wild et al. 2010; McAlpine et al. 2020) between the onset of the actual phase of active black hole growth and the beginning/coalescence of the merger. Hence, from the perspective of timescales our result solely depends on the relative difference between the AGN and merger lifetimes and thus we have to consider our fraction of inactive merging galaxies, which have hosted an AGN to be an upper limit. However, a visual re-examination returned only a low number of galaxies with asymmetries actually having a close companion. Therefore, we conclude that most of the distorted galaxies are already in the late stages of their merging process, indicating that, if at all, they already experienced a potential AGN phase with a low chance of an intermittent AGN becoming active again.

The total merger fraction of our inactive galaxies, which amounts to $f_{\mathrm{m} \text {,ina }} \sim 0.35$, serves as an upper bound for $f_{\mathrm{m} \text {,ina \& agn }}$. Both parameters being equal would imply that all distorted, inactive galaxies have hosted (or will host) an AGN. Conversely, $f_{\mathrm{m} \text {,ina \& agn }}=$ 0 would correspond to no such galaxy ever hosting an AGN. In Fig. 6 we present the results of our computations for different $f_{\mathrm{m} \text {,agn }}$ and $t_{\mathrm{m}}=10^{9} \mathrm{yr}$ (left) and $1.5 \times 10^{9} \mathrm{yr}$ (right). The blue lines and the shaded regions denote the results for our retrieved AGN merger fraction and the corresponding $1 \sigma$ intervals, while the violet and yellow lines display the trend for $f_{\mathrm{m}, \mathrm{agn}}=0.30$ and 0.70 , respectively. The fraction of merging inactive galaxies hosting an AGN at some point during the merging process increases with shorter AGN lifetimes. In addition, for a given period of AGN activity this share grows with longer merger timescales and larger AGN merger fractions, both due to an enhanced probability to find a distorted galaxy actually hosting an AGN. Depending on the merger timescale and assuming the lower limit of our AGN merger fraction is correct, we can deduce a lower bound for the AGN lifetime by considering every inactive distorted galaxy to host an AGN, i.e. $f_{\mathrm{m} \text {,ina \& agn }} \equiv f_{\mathrm{m} \text {,ina }}$. The life span of an AGN corresponds then to a minimum of $1.3 \times 10^{6} \mathrm{yr}$ and $1.9 \times 10^{6} \mathrm{yr}$ for merger timescales of $10^{9} \mathrm{yr}$ and $1.5 \times 10^{9} \mathrm{yr}$, respectively (Fig. 6, dotted lines).

However, based on the best estimates for accretion rate histories we have today (Di Matteo et al. 2005; Johansson et al. 2009a,b; Hopkins \& Quataert 2010; Jung et al. 2018), we fix the time period in which an AGN accretes above $\lambda_{\text {edd }}>0.3$ to $t_{\text {agn }}=10^{7} \mathrm{yr}$. The inferred fractions of inactive merging galaxies that also host an AGN at any given time yield then $f_{\mathrm{m} \text {,ina \& agn }}=$ $0.06_{-0.02}^{+0.01}$ and $0.09_{-0.02}^{+0.02}$ for $t_{\mathrm{m}}=10^{9} \mathrm{yr}$ and $1.5 \times 10^{9} \mathrm{yr}$, respectively (Fig. 6, dashed lines). So, adding even the upper limit of this fraction onto the AGN major merger rate we derived in Section 3.1 this only results in a revised AGN major merger fraction, which is barely above the threshold of 0.5 , which in turn would indicate that the majority of AGNs is triggered by major mergers. This result still leaves $\sim 50 \%$ of AGNs to be of unknown origin. Only by assuming a significantly lower AGN duty cycle of $t_{\text {agn }} \sim 10^{6} \mathrm{yr}$ and thus regarding almost every distorted inactive galaxy hosting an AGN, we can obtain AGN major merger fractions of $\sim 80 \%$, 

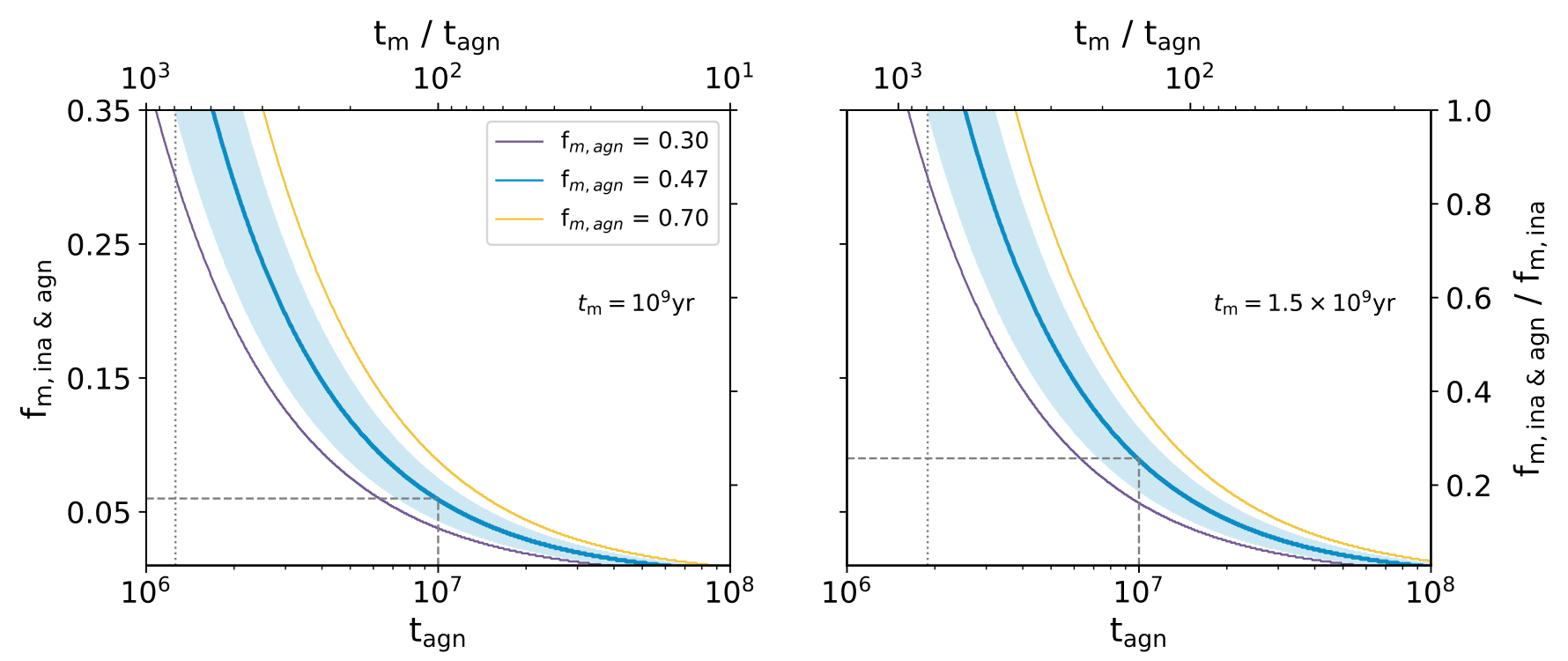

Figure 6. Total fraction of merging inactive galaxies that hosted an AGN in the recent past $f_{\mathrm{m} \text {,ina \& agn }}$ in dependence of the AGN life time $t_{\mathrm{agn}}$ for a merger timescale $t_{\mathrm{m}}$ of $10^{9} \mathrm{yr}$ (left) and $t_{\mathrm{m}}=1.5 \times 10^{9} \mathrm{yr}$ (right). The blue line including the shaded region represents our result of the AGN total merger fraction of $f_{\mathrm{m} \text {,agn }}=0.47 \pm 0.12$. The violet and yellow lines correspond to $f_{\mathrm{m}, \text { agn }}=0.30$ and 0.70 , respectively. The dotted line corresponds to a lower limit of $t_{\mathrm{agn}}$, the dashed lines display the resulting $f_{\mathrm{m} \text {,ina \& agn }} \sim 0.09$ for an assumed $t_{\mathrm{agn}}=10^{7}$.

which would then leave no doubt about the role of major mergers and the triggering of high Eddington rate AGNs at $z<0.2$. Hence, we conclude that neither a difference in AGN and merger timescales nor the potential presence of intermittent AGNs affect significantly our derived AGN merger rate. In order to better constrain our inferred estimates, more detailed simulations predicting especially AGN timescales in dependence of accretion rate are imperative.

\section{SUMMARY \& CONCLUSIONS}

We examined a potential direct connection between AGNs specifically exhibiting the highest Eddington ratios and major mergers at $z<0.2$. We analyzed 17 AGN host galaxies and 25 comparison galaxies, matched in mass, redshift, filter, and the $\mathrm{S} / \mathrm{N}$ in $V, B$ and color images. We adjusted our control galaxies by adding artificial point sources on top of their flux centers, which yielded two indistinguishable samples, that were joined to create a randomized overall sample of 42 targets. This overall sample was ranked according to the presence of merger features (from most to least distorted) by 19 experts. We combined the individual rankings of each set, i.e. $V, B$ and color, by applying three different methods, resulting in a total number of nine consensus rankings. This allowed us to determine any bias, which might be introduced by visually classifying the galaxies at different wavelengths or the algorithm to combine the individual classifications. Finally, we also created one overall sequence by combining the nine initial consensus rank- ings. We divided all rankings into; 1) galaxies showing distinct merger features and 2) galaxies showing no signs of a gravitational disturbance, by choosing specific cut-off ranks. As a final step we derived the respective merger fractions by counting the numbers of active and control galaxies above and below these particular limits and applying those quantities to a beta distribution.

Our findings depend heavily on the choice of distinction between merging and undisturbed systems. To analyze how the selection of the cut-off rank affected our result, we; (1) selected it based on the visual interpretations by the experts and (2) chose it such that the merger rate of our comparison sample was consistent with the overall major merger fraction of galaxies in our mass and redshift range. When we considered the average determinations of the classifiers, approximately half of both populations showed signs of a current or recent merger event, suggesting no causal connection between major mergers and the triggering of this particular population of AGNs.

Since our first approach also considers asymmetries or signatures that stem from processes other than a major merger event, we adjust the major merger fraction of the inactive galaxies to be consistent with recent simulations and observations. As a result we find a substantial excess in the major merger fraction of the AGN sample with respect to the inactive galaxies. Coincidentally, with a separation at the corresponding cut-off rank we also found a clear distinction between strongly- 
disturbed galaxies and galaxies with either minor or no merger signatures, confirming our classification.

We summarize our findings as follows.

- The merger fractions of the AGN host galaxies and comparison galaxies are $f_{\mathrm{m} \text {,agn }}=0.41 \pm 0.12$ and $f_{\mathrm{m}, \text { ina }}=0.08 \pm 0.06$, respectively.

- Neither the choice of set nor combination method has impacts the recovered merger fractions.

- For our AGNs, with the highest Eddington ratios at $z<0.2$, major mergers are an essential mechanism to trigger black hole growth.

- We rule out that minor mergers play a considerable role in the triggering of our subpopulation of AGNs.

- Considering AGN and merger lifetimes as well as AGN variability induced by an ongoing merger event, our best estimate results in $\sim 50 \%$ of our AGN population still being of unknown origin.

Extending our study to include IFU-observations and a larger number of sources would enable us to analyze the AGN host galaxies in more detail. By assessing the strength of potential past merger events by examining the kinematics and stellar populations, while larger number provides better statistics we can determine, which processes are responsible for the triggering of the remaining $\sim 50 \%$ and whether major mergers are indeed the dominant mechanism.

\section{ACKNOWLEDGMENTS}

We thank the referee for the constructive feedback, which improved the quality of this work.

We also thank Mischa Schirmer for his helpful guidance in using THELI.

JS is supported by JSPS KAKENHI Grant Number JP18H01251 and the World Premier International Research Center Initiative (WPI), MEXT, Japan. VNB gratefully acknowledges assistance from a National Science Foundation (NSF) Research at Undergraduate Institutions (RUI) grant (AST-1909297). Note that findings and conclusions do not necessarily represent views of the NSF. RAW acknowledges support from NASA JWST Interdisciplinary Scientist grants NAG5-12460, NNX14AN10G and 80NSSC18K0200 from GSFC.

Based on observations made with ESO Telescopes at the La Silla Paranal Observatory under program ID 091.B-0672, 095.B-0773 \& 098.A-0241.

Funding for the Sloan Digital Sky Survey (SDSS) has been provided by the Alfred P. Sloan Foundation, the
Participating Institutions, the National Aeronautics and Space Administration, the National Science Foundation, the U.S. Department of Energy, the Japanese Monbukagakusho, and the Max Planck Society. The SDSS Web site is http://www.sdss.org/.

The SDSS is managed by the Astrophysical Research Consortium (ARC) for the Participating Institutions. The Participating Institutions are The University of Chicago, Fermilab, the Institute for Advanced Study, the Japan Participation Group, The Johns Hopkins University, Los Alamos National Laboratory, the Max Planck Institute for Astronomy (MPIA), the Max Planck Institute for Astrophysics (MPA), New Mexico State University, University of Pittsburgh, Princeton University, the United States Naval Observatory, and the University of Washington.

This research has made use of NASA's Astrophysics Data System Bibliographic Services.

\section{Facility: ESO-VLT(FORS2)}

Software: astropy (Astropy Collaboration et al. 2013, 2018), Matplotlib (Hunter 2007), MultiColorFits (Cigan 2019), SAOImageDS9 (Joye \& Mandel 2003), THELI (Erben et al. 2005; Schirmer 2013) 


\section{APPENDIX}

\section{A. DETAILS ON COMBINATION METHODS}

Every method to combine individual votes into a combined consensus sequence violates at least one of three criteria described by Arrow's Impossibility Theorem (Arrow 1950). It states that no existing method, which combines two or more individual votes satisfies the following three axioms: (1) non-dictatorship, such that all individual votes are considered to be equal; (2) unanimity or the weak Pareto principle, stating that if all voters agree on $X>Y$, this also holds true for the overall ranking; and (3) the independence of irrelevant alternatives, such that the consensus relation between $X$ and $Y$ only depends on the individual preferences between those two entities and not any additional option(s). As additional conditions we introduce the Condorcet paradox and the Condorcet criterion (Condorcet 1785; Condorcet et al. 1989). The first one states that an overall sequence can be cyclic - e.g. $X$ wins over $Y$, which wins over $Z$, which in turn wins over $X$ - although the individual votes are not. The latter explains that an overall top-ranked candidate wins in every pairwise comparison with every other candidate.

Below we present the methods we apply to create the overall rankings. As stated in Section 3 we use three different algorithms to construct those combined rankings to determine any potential bias introduced by the method. However, in addition all of our three methods also satisfy or infringe the above mentioned criteria differently, which gives us even more detailed insights in any potential introduction of differences in the merger fractions.

For our first method to combine the individual expert rankings we adopt the same method applied in Mechtley et al. (2016) and Marian et al. (2019). We start with calculating the mean rank for each galaxy from the individual rankings and discard every individual expert classification of each galaxy, if it differs more than $2 \sigma$ from the respective average rank. Out of the 798 individual assessments in $V$-band we reject 25 votes, while out of the total 779 ratings, 17 are discarded for the sets in $B$-band and color, respectively. However, since we weigh individual votes this method obviously violates the non-dictatorship criterion.

Our second method, the Borda count approach (Emerson 2013), satisfies this condition, but violates in exchange the independence of irrelevant alternatives. We adapt the original version of this method in which the first ranked option receives $n$ points, the second one $n-1$ and so on, with $n$ being the total number of candidates, by applying the Dowdall system (Reilly 2002). With that approach the candidates receive the reciprocal value of their respective ranks, i.e. the first ranked option is rewarded $1 / n=1$ point, the next one 0.5 points and so on. As low rank galaxies may be ranked more randomly due to a lack of significant merger features, we can decrease the impact those sources might have on our overall ranking by using this variant of the Borda count.

This approach avoids the Condorcet paradox, but only our third method, the Schulze method (Schulze 2011, 2018), also satisfies the Condorcet criterion. With this method all pairwise comparisons between two candidates $X$ and $Y$ for all individual rankings are calculated and put into relation to each other, resulting in an overall ranking, where the top-ranked candidate, wins indeed over all other candidates, being the so-called Condorcet winner. Going to lower ranks within the resulting consensus sequence the second-placed candidate only loses to the first-ranked option and so on (for more details and examples please see Schulze 2018).

\section{B. DEPENDENCE OF MERGER FRACTIONS ON CUT-OFF RANK}

In Sections 3 and 4 we describe how the choice of cut-off rank can influence the resulting merger fractions and also present for four selected cut-off ranks the corresponding merger fractions. In Figure 7 we now present the continuous dependence of merger fractions on cut-off rank for all combinations of method and set. The AGN host galaxies and inactive galaxies are shown in blue and red, respectively. The shaded regions denote the $1 \sigma$ confidence interval from shot- and classification-noise. As already indicated in Figure 3 and described in Section 3, it is also shown in Figure 7 that first, neither the choice of method to combine the individual rankings nor the selection of set has any significant impact on the resulting absolute merger fractions or the relative differences between them. Second, compared to the inactive comparison sample and for cut-off ranks $\lesssim 15$ the AGN host galaxies show a clear excess in merger fractions. This clearly indicates that our conclusions rely considerably on the choice of cut-off rank, which is extensively discussed in the main text. 


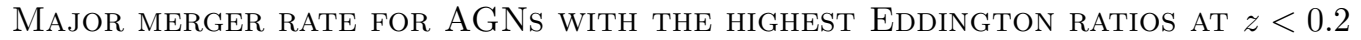
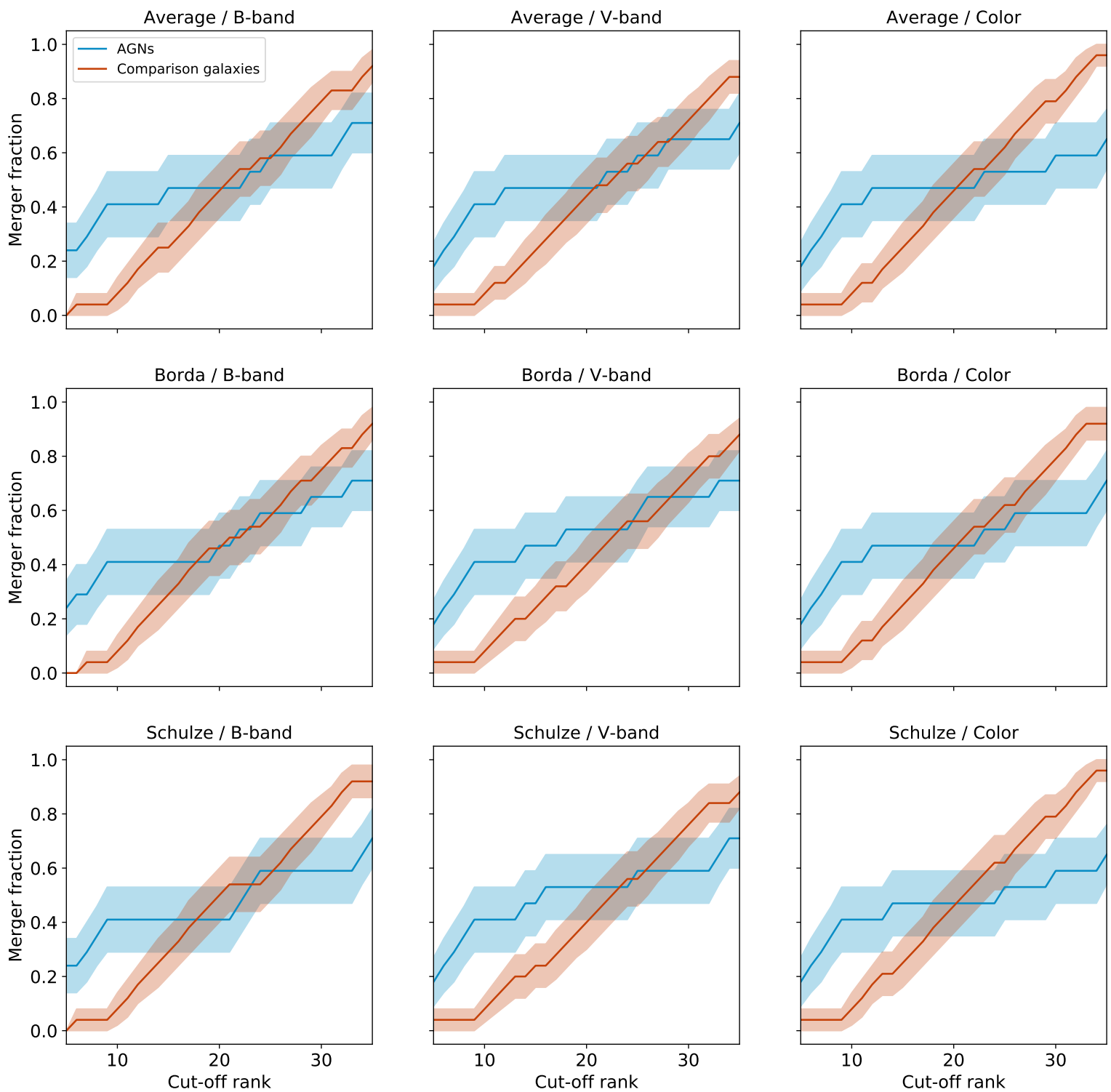

Figure 7. Evolution of the merger fractions for the AGN host galaxies (blue) and inactive galaxies (red) in dependence of cut-off rank for each combination of set and method. The shaded regions give the $1 \sigma$ confidence interval. 


\section{VISUAL OVERALL CONSENSUS RANKING}

To have a 'meta' singular consensus sequence we apply the Schulze method (see Section 3 and Appendix A) to our final nine overall rankings, which we calculated for each combination of set and method. We show all sources in the resulting order, and include for completeness also the sources already shown in Figure 2. The respective rank for each object is given in parentheses besides its designation. It should be noted that Gal176221 is only ranked last, because it was only observed in $V$-band and therefore only appears in the three corresponding consensus rankings. In those three respective rankings it is always positioned at rank 14. Clearly visible is the drop-off in strong merger features at a cut-off rank $\gtrsim 10$.

\section{(1) - SDSSJ105007.75+113228.6}

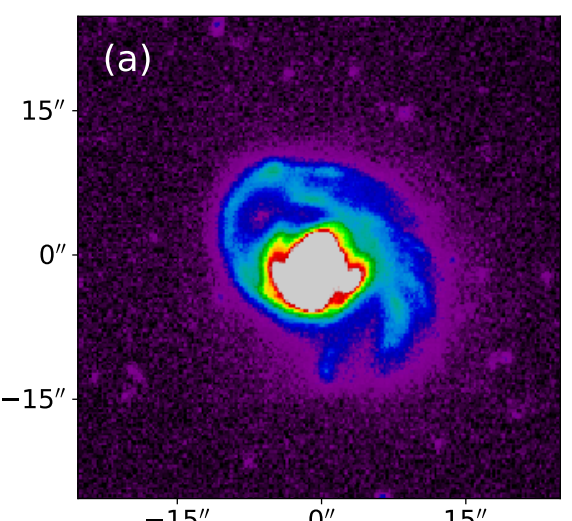

$-15^{\prime \prime}$

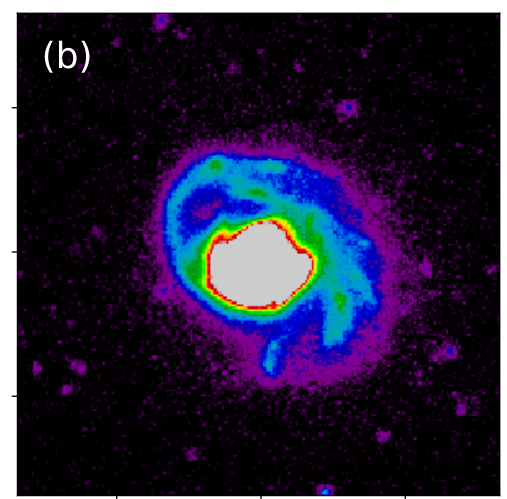

$-15^{\prime \prime}$

$15^{\prime \prime}$

\section{(2) - Gal030481}

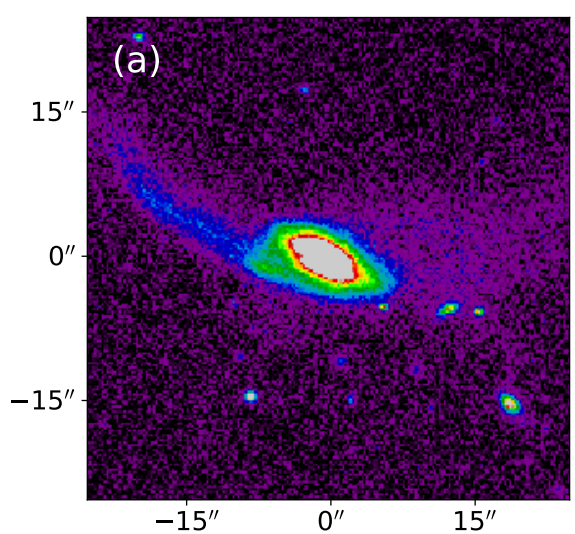

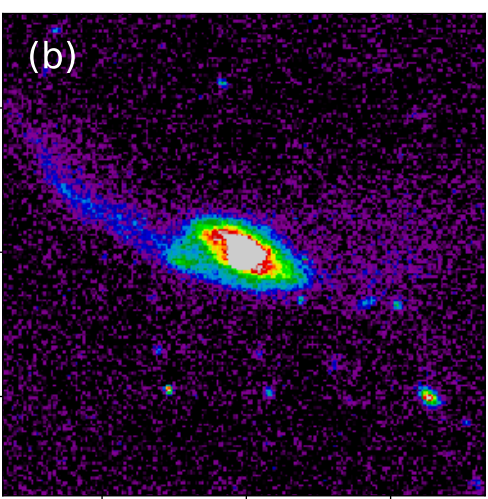

$-15^{\prime \prime}$

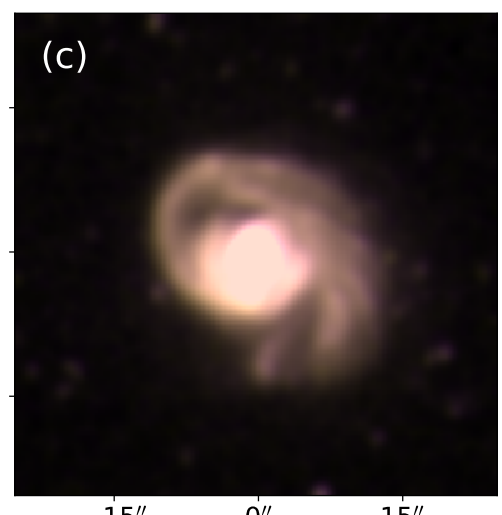

$-15^{\prime \prime}$

$15^{\prime \prime}$

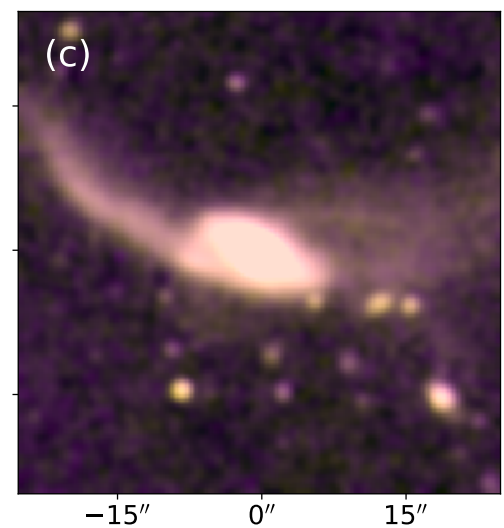

$15^{\prime \prime}$

Figure 8. From left to right we present a postage stamp in (a) V-band, (b) B-band and (c) color, respectively. Note: In order to enhance the visibility the images are not shown with the same cuts and color map parameters. 
(3) - HE0157+0009
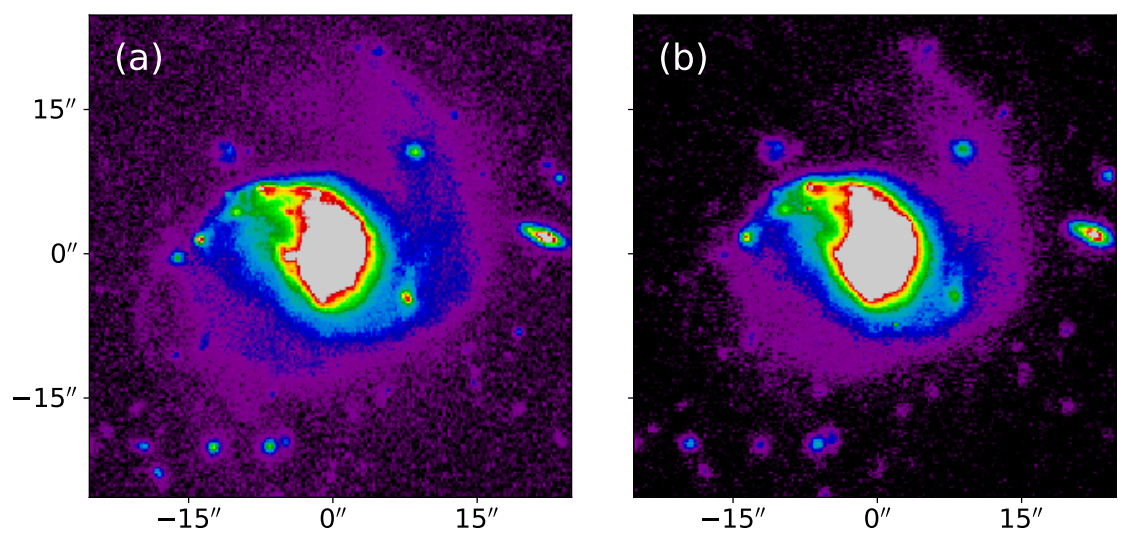

(4) - HE2011-6103
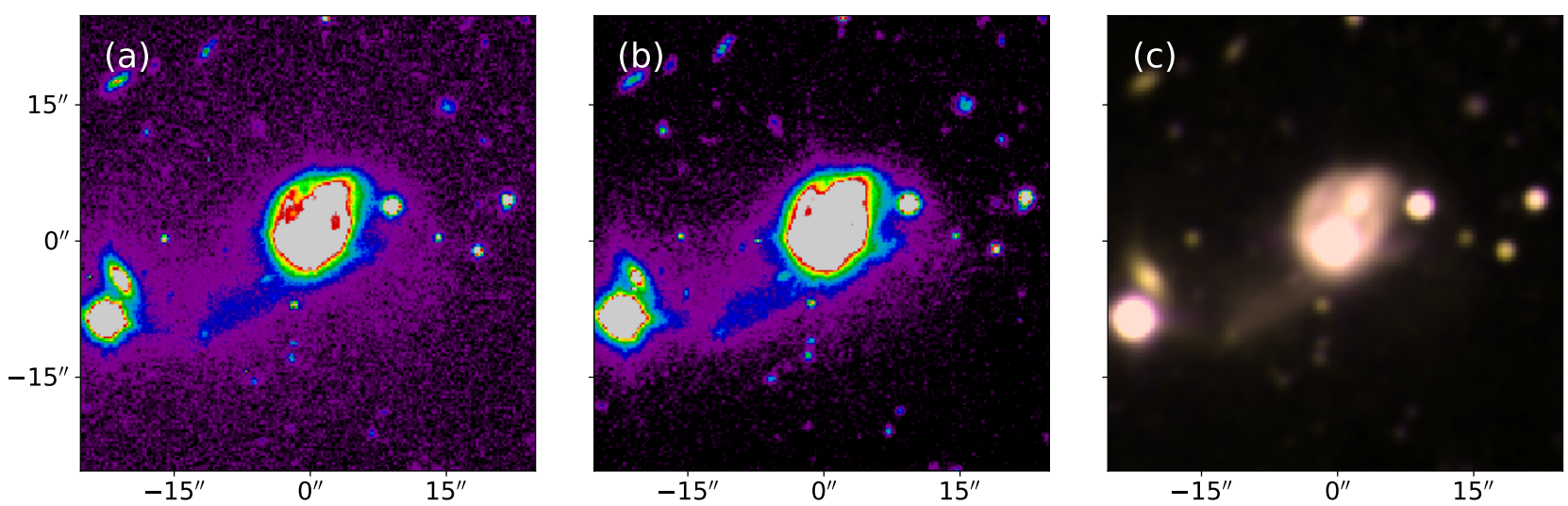

(5) - HE2258-5524

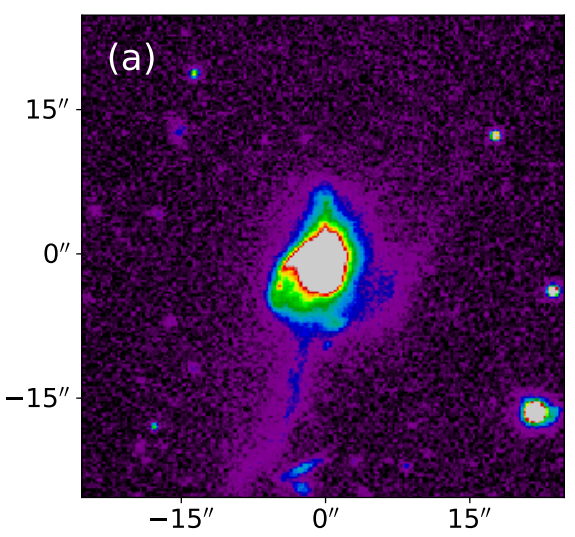

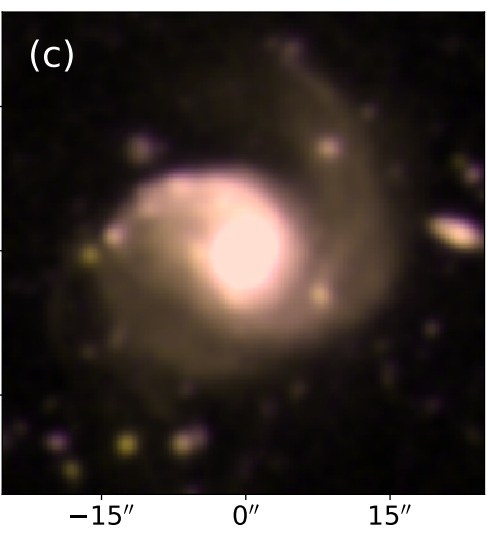

(b)

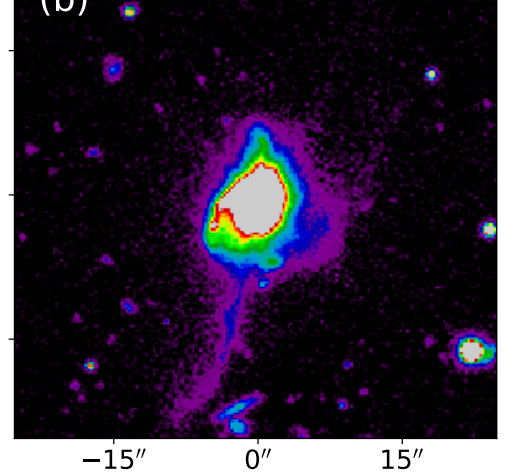

Figure 8. (Continued.)

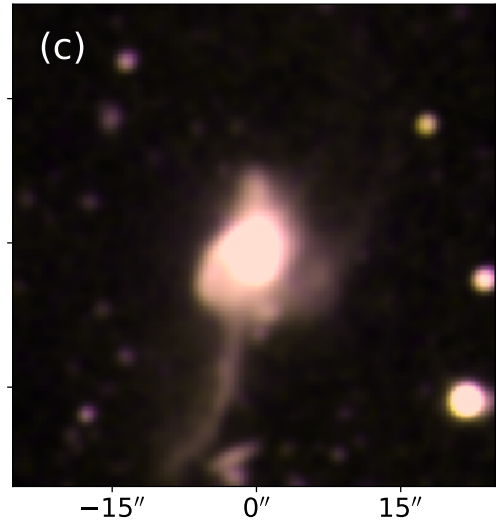


(6) - HE0132-0441
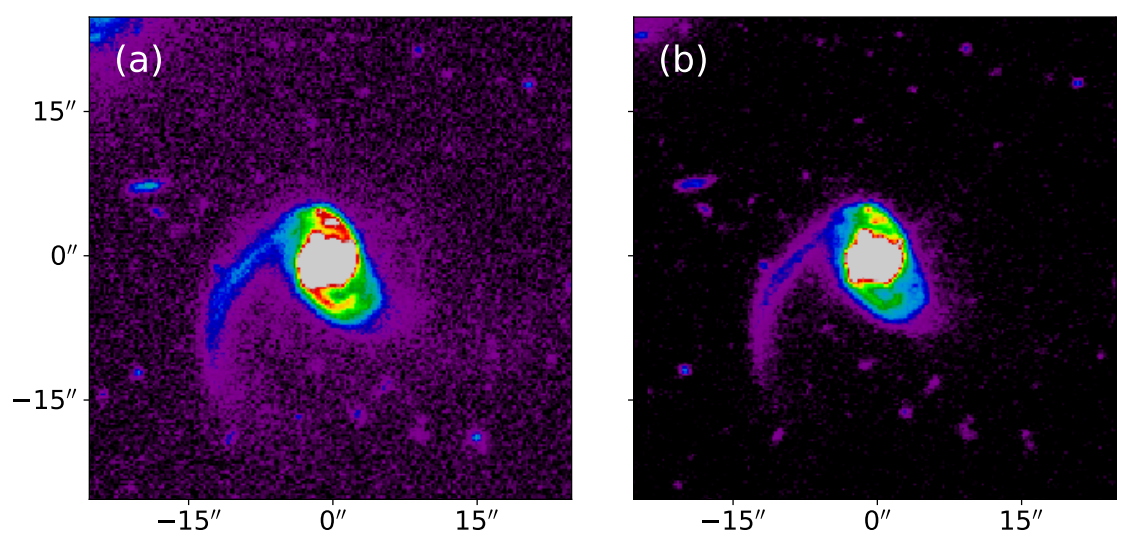

(7) - HE0558-5026

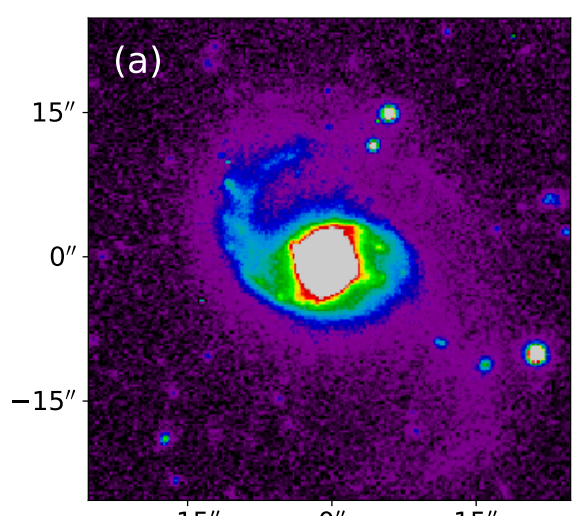

$-15^{\prime \prime}$

$0^{\prime \prime}$

$15^{\prime \prime}$

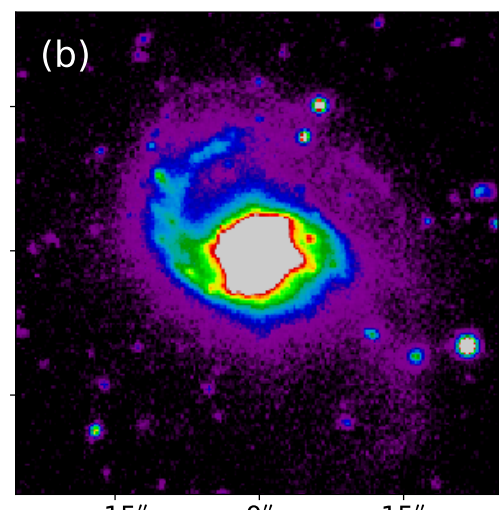

$-15^{\prime \prime}$

$15^{\prime \prime}$

(8) - PG1012+008

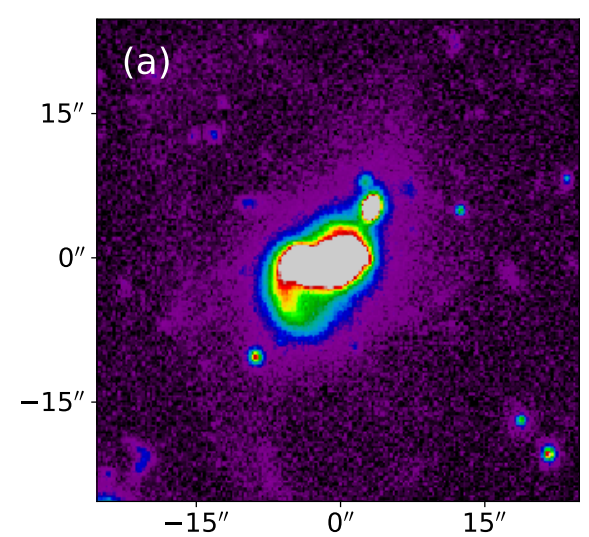

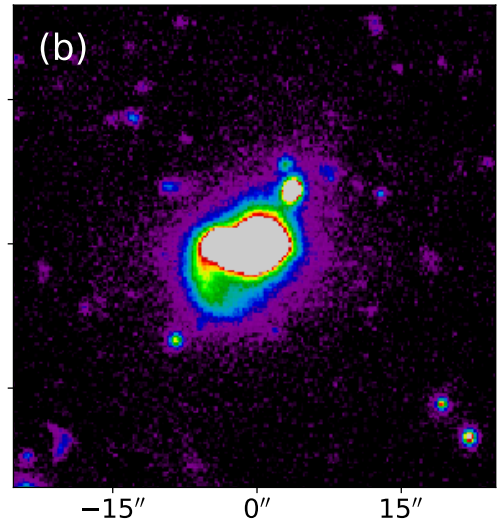

Figure 8. (Continued.)
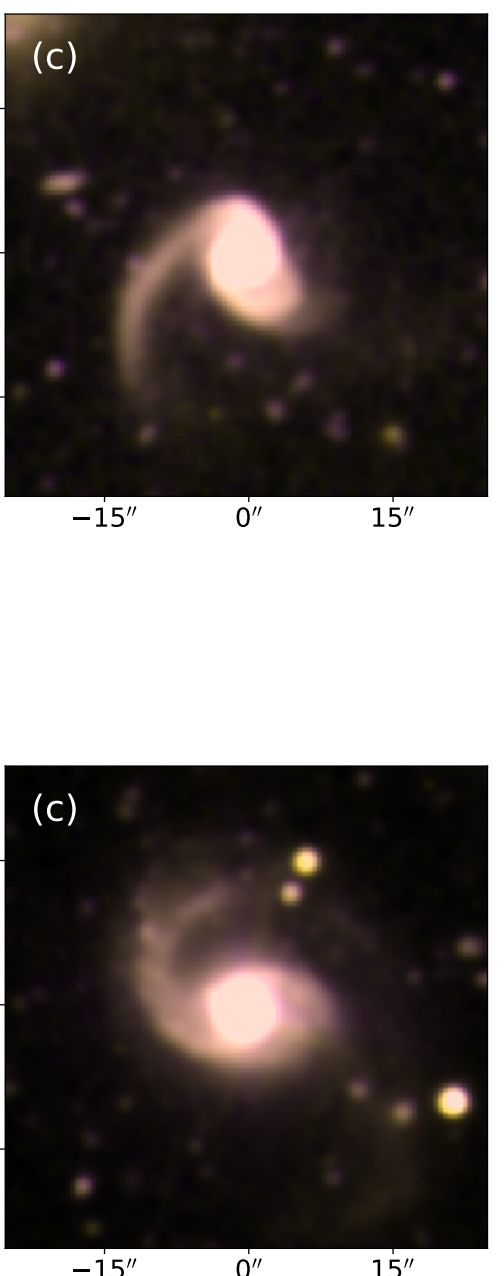

$-15^{\prime \prime}$

$15^{\prime \prime}$

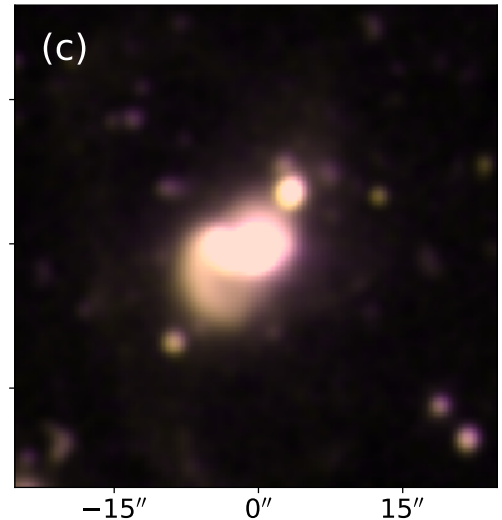

$-15^{\prime \prime}$ 


\section{(9) - Gal458007}
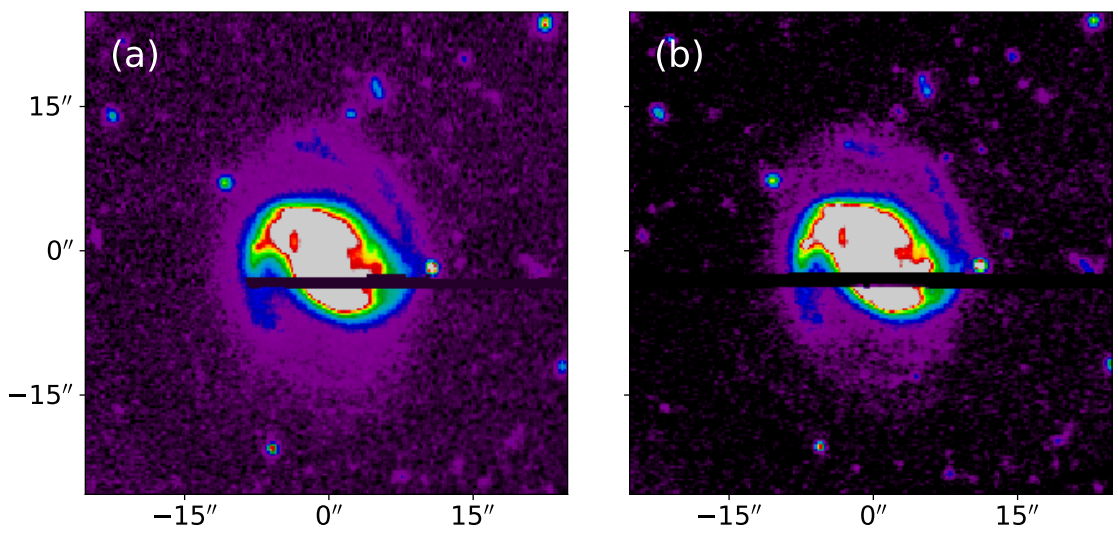

(10) - Gal079769

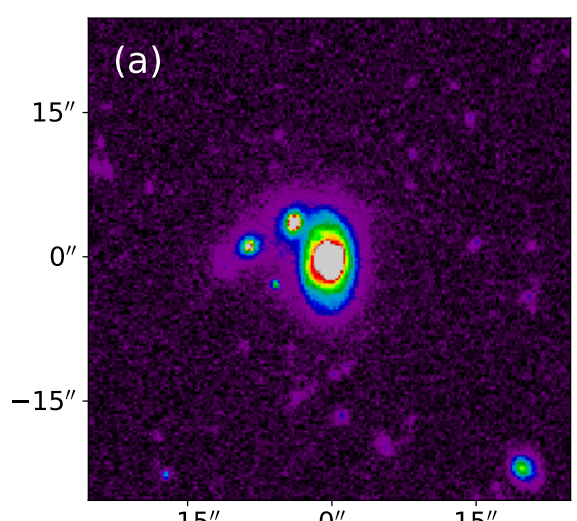

$-15^{\prime \prime}$

$15^{\prime \prime}$

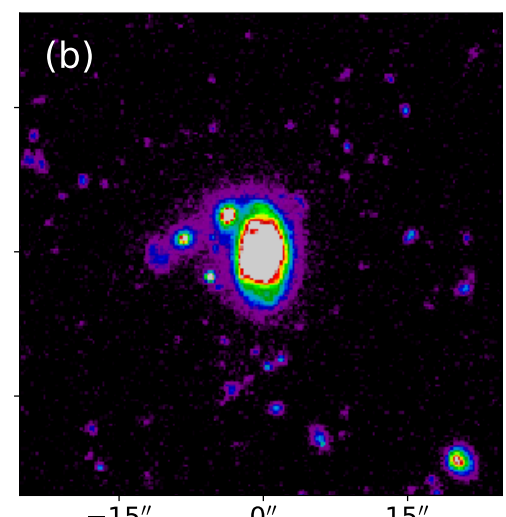

(11) - Gal270096

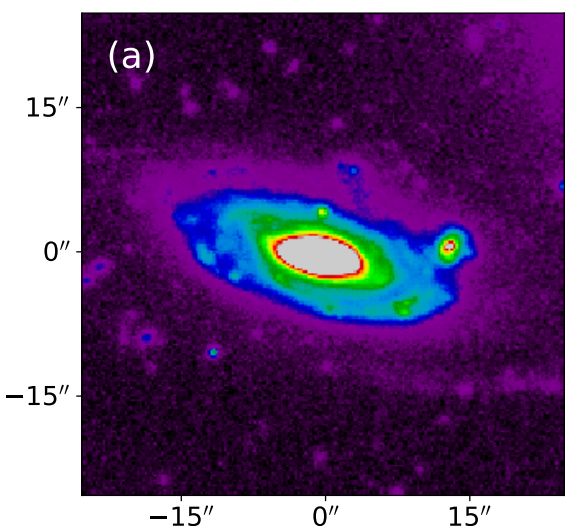

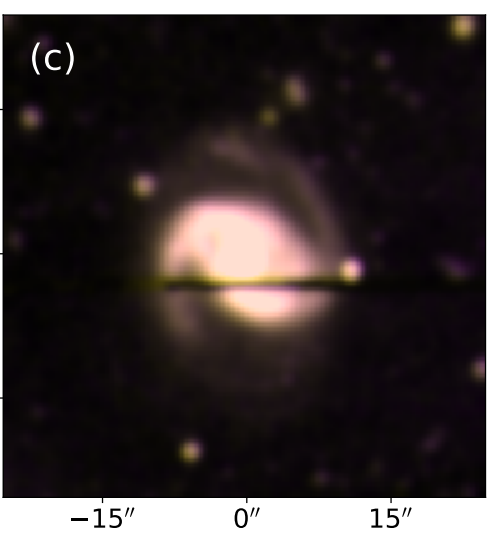
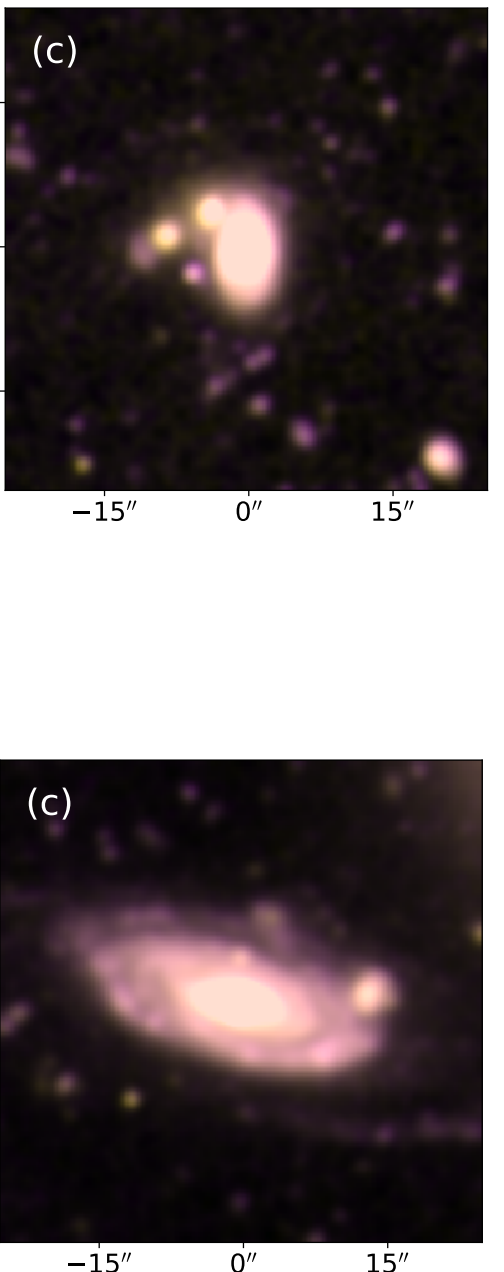

Figure 8. (Continued.) 
(12) - Gal698144
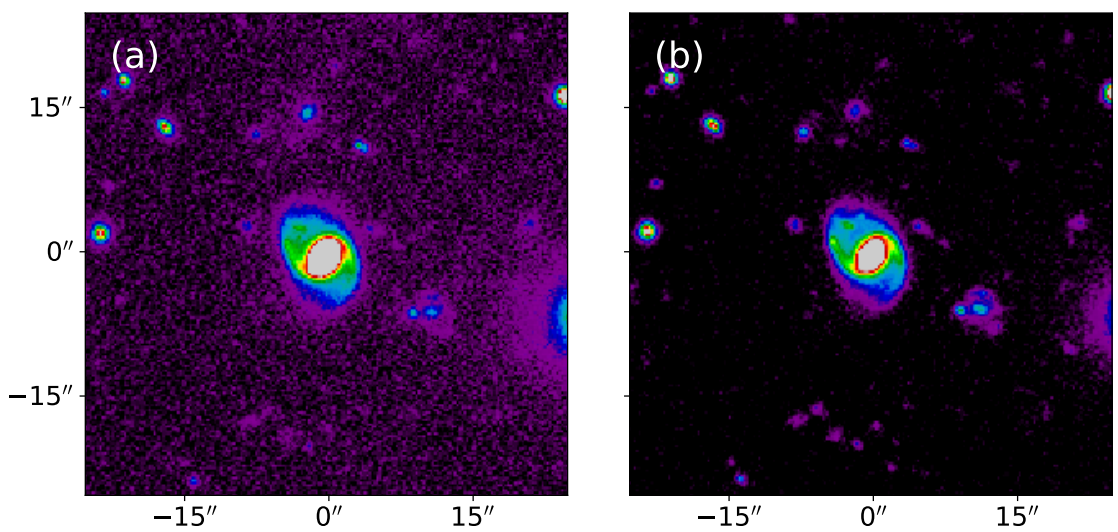

(13) - Gal782980

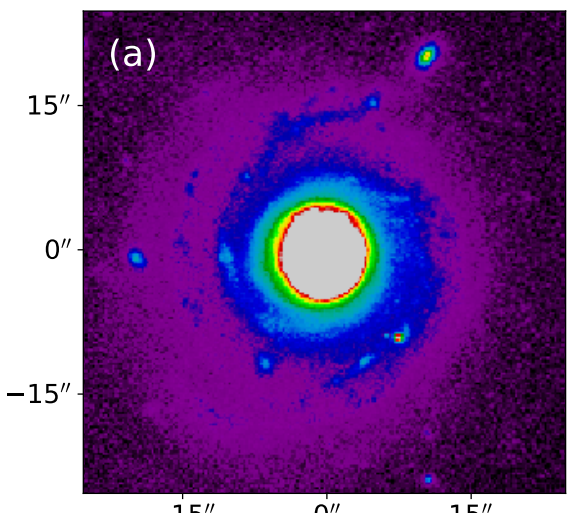

$-15^{\prime \prime}$

$15^{\prime \prime}$

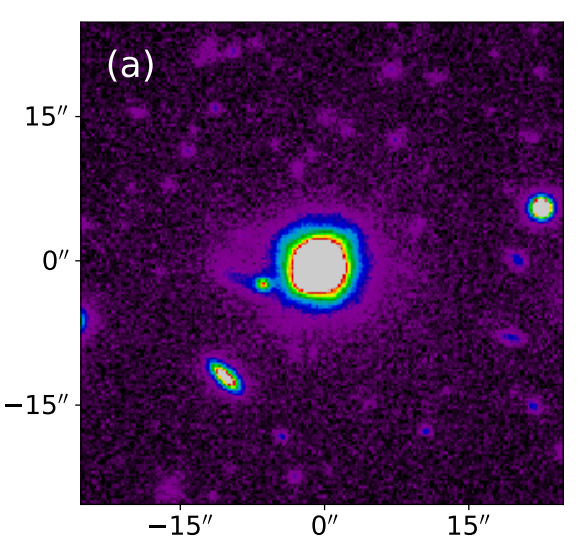

(14) - HE0444-3449

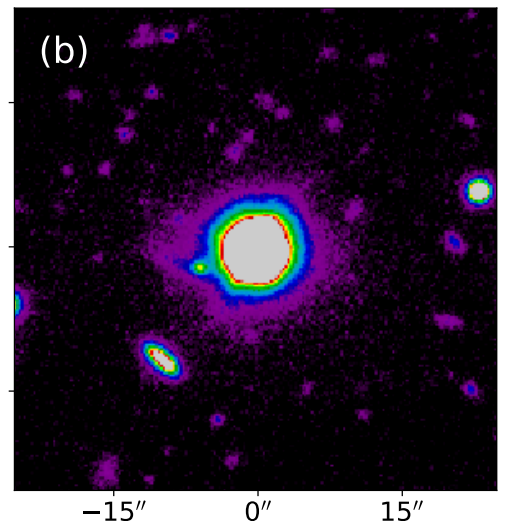

Figure 8. (Continued.)

(5

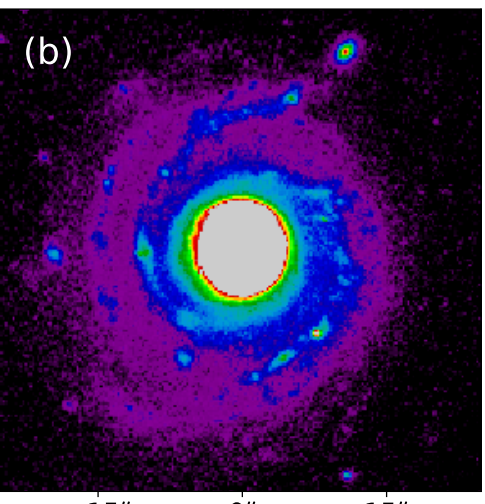

$-15^{\prime \prime}$

$5^{\prime \prime}$
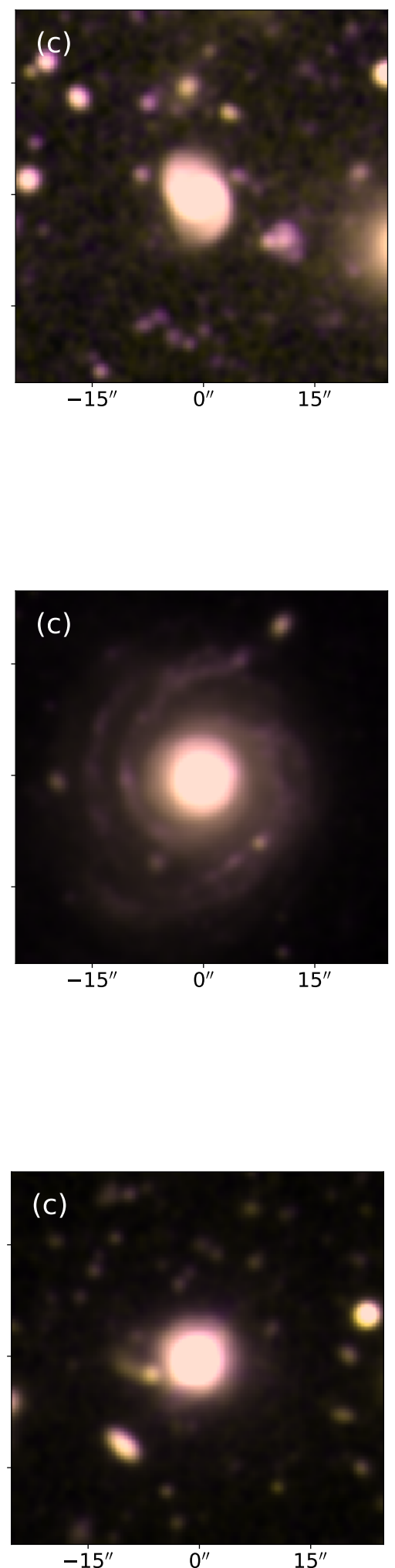

$-15$ $0^{\prime \prime}$ 
(15) - Gal534882
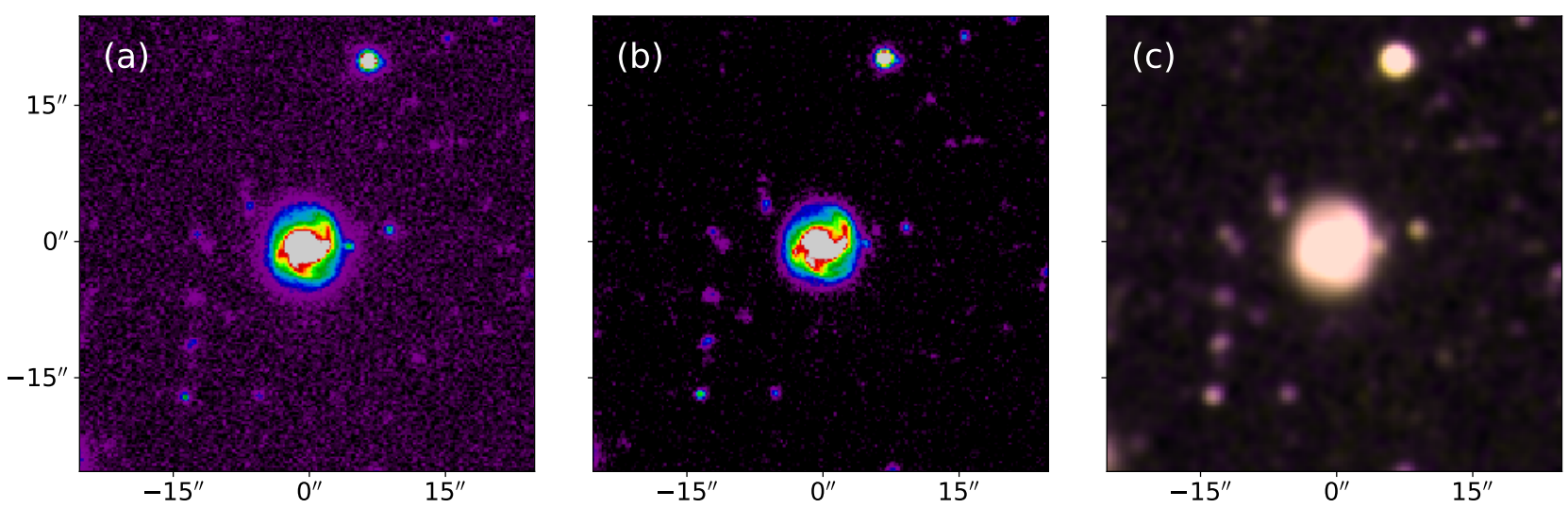

(16) - Gal510223
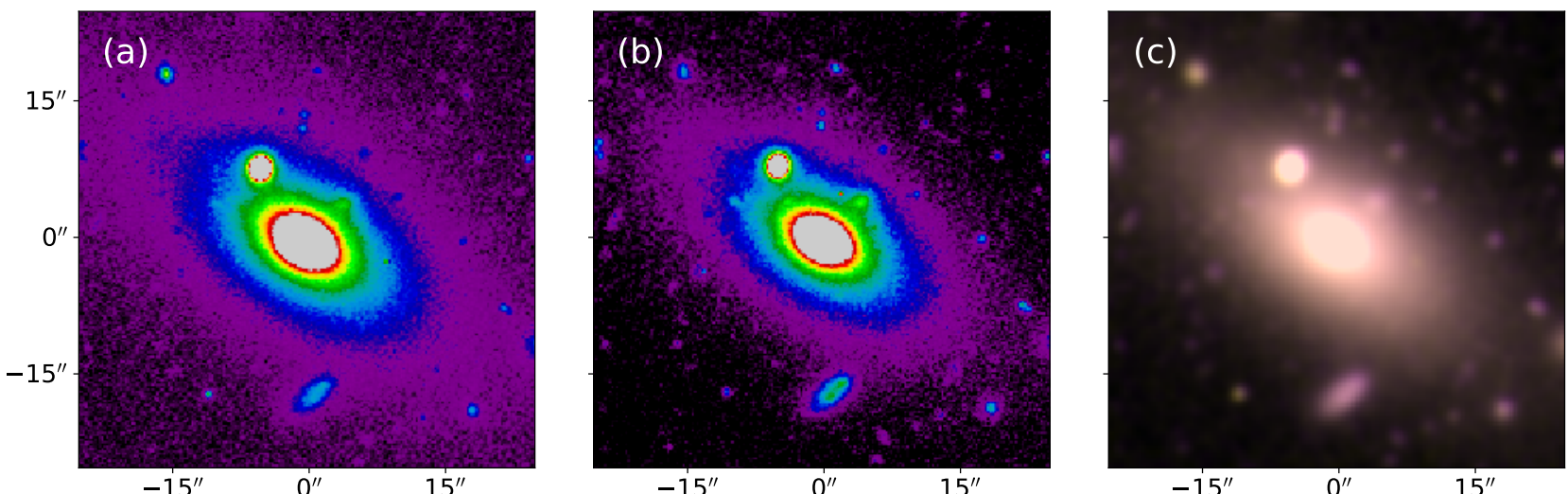

(17) - Gal050873
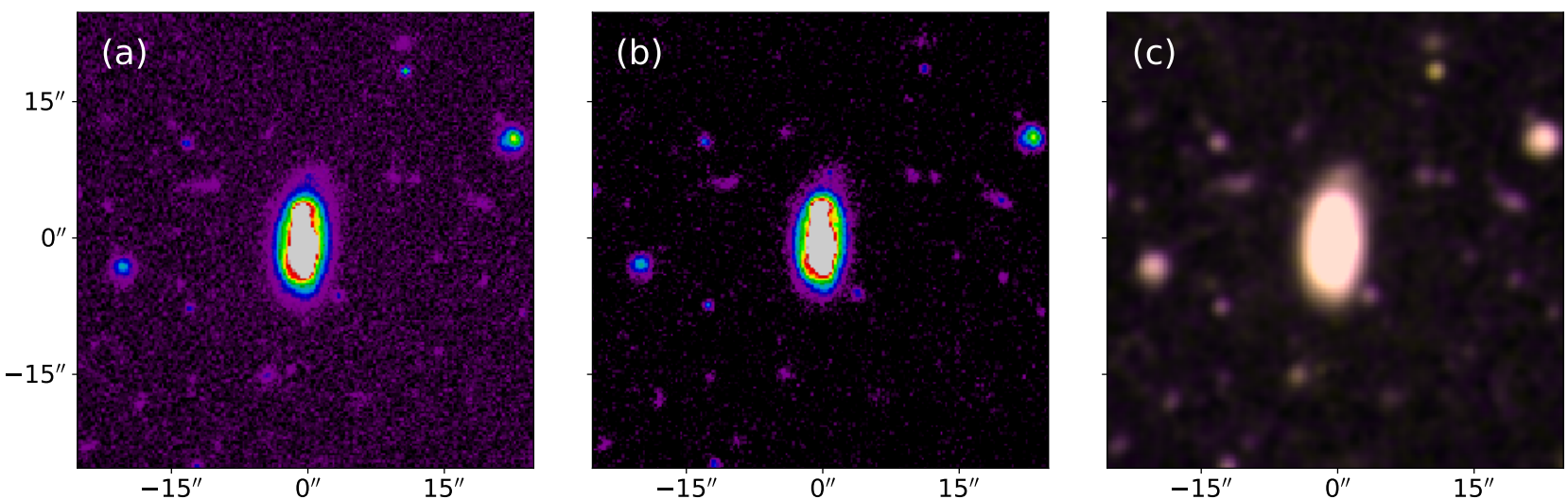

Figure 8. (Continued.) 
(18) - Gal419090
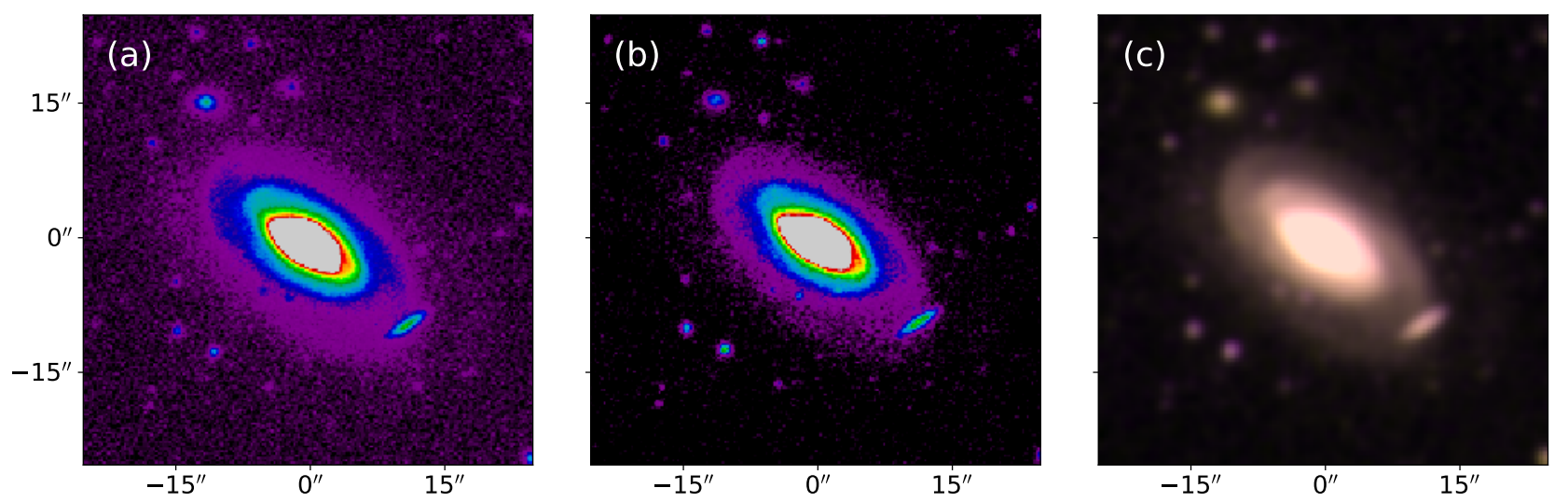

(19) - Gal676011

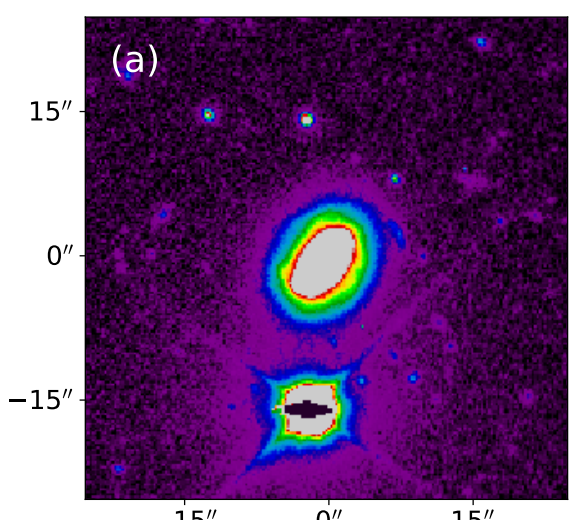

$-15^{\prime \prime}$

$0^{\prime \prime}$

$15^{\prime \prime}$

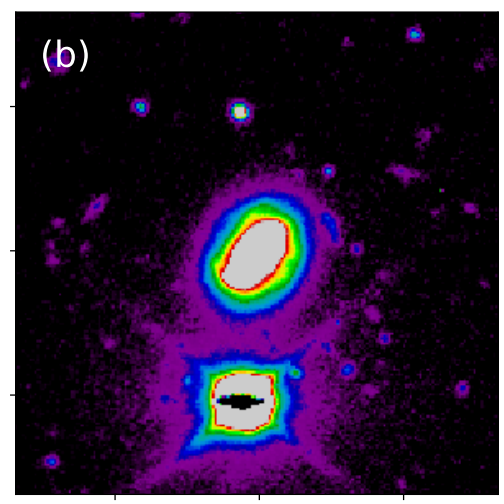

$-15^{\prime \prime}$

$15^{\prime \prime}$

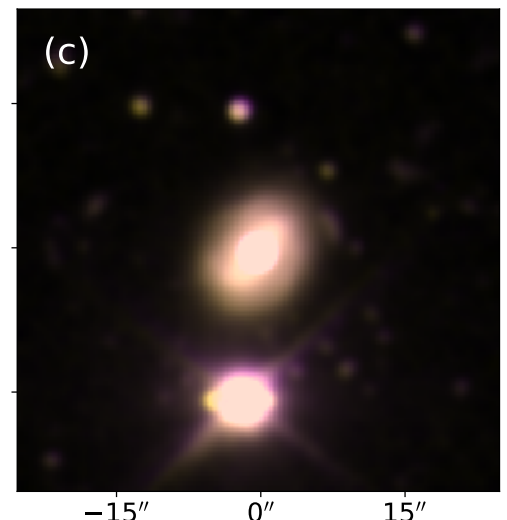

$-15^{\prime \prime}$

$15^{\prime \prime}$

(20) - SDSSJ124341.77+091707.1

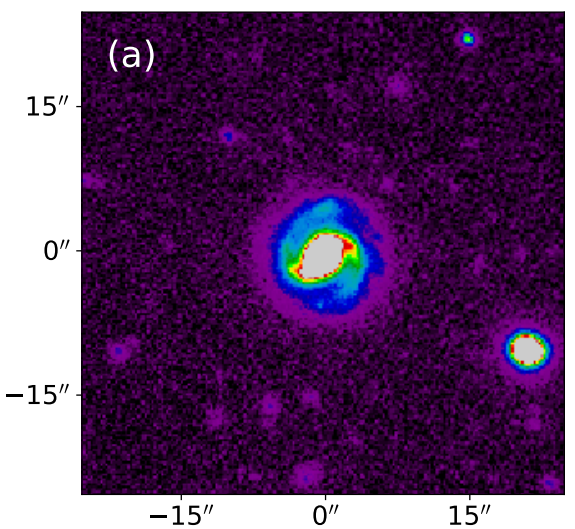

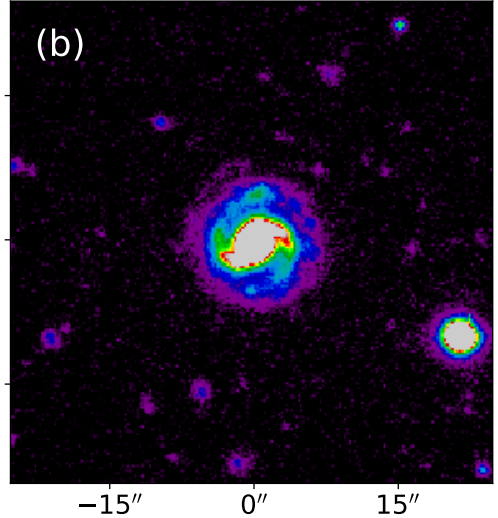

Figure 8. (Continued.)

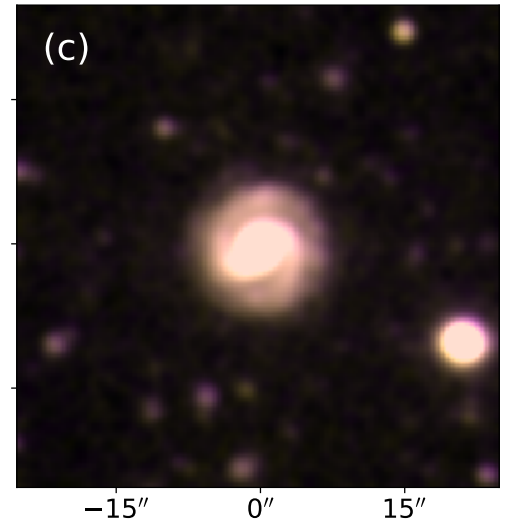

$-15^{\prime \prime}$ 
(21) - Gal498251
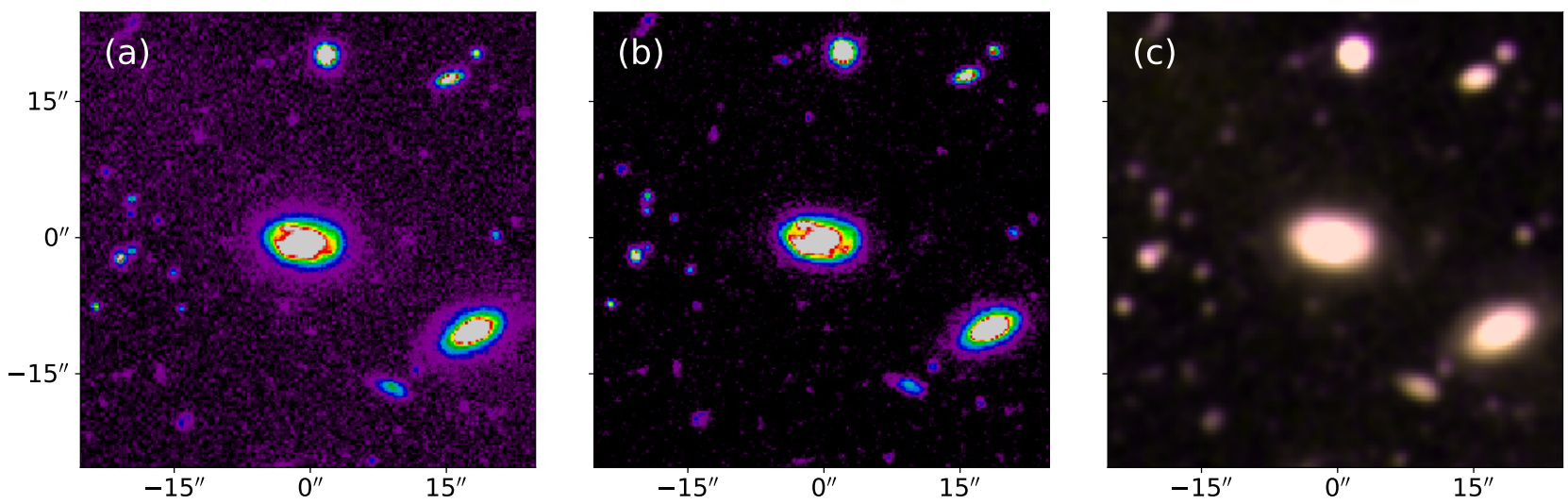

(22) - Gal286443
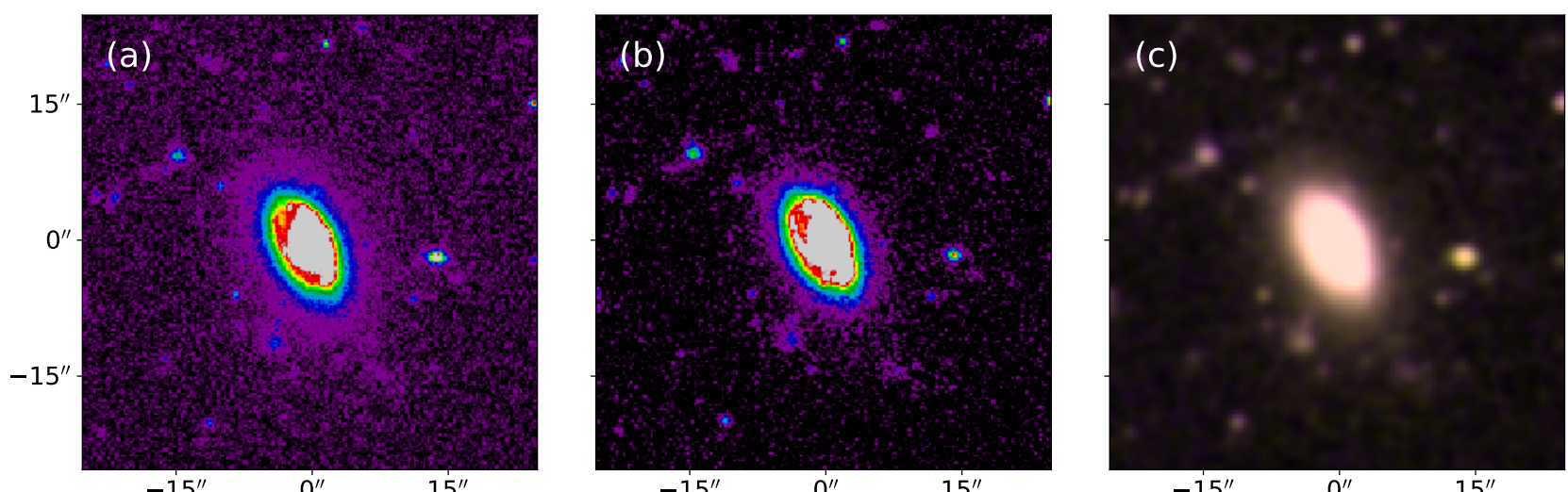

(23) - HE2152-0936
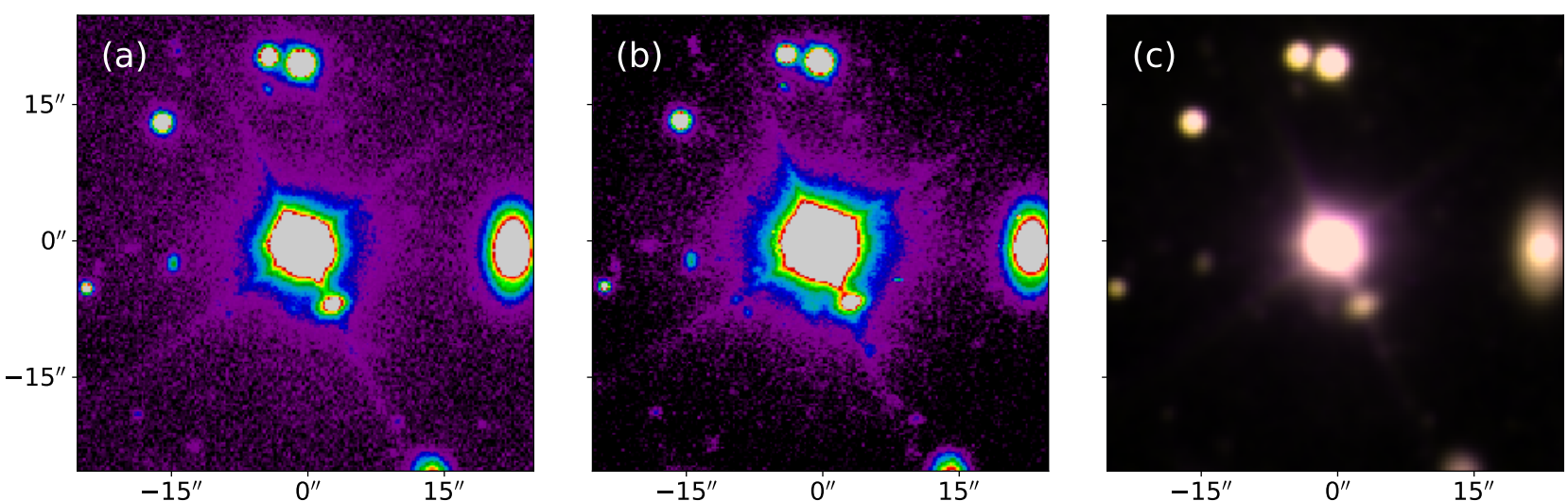

Figure 8. (Continued.) 
(24) - Gal185580
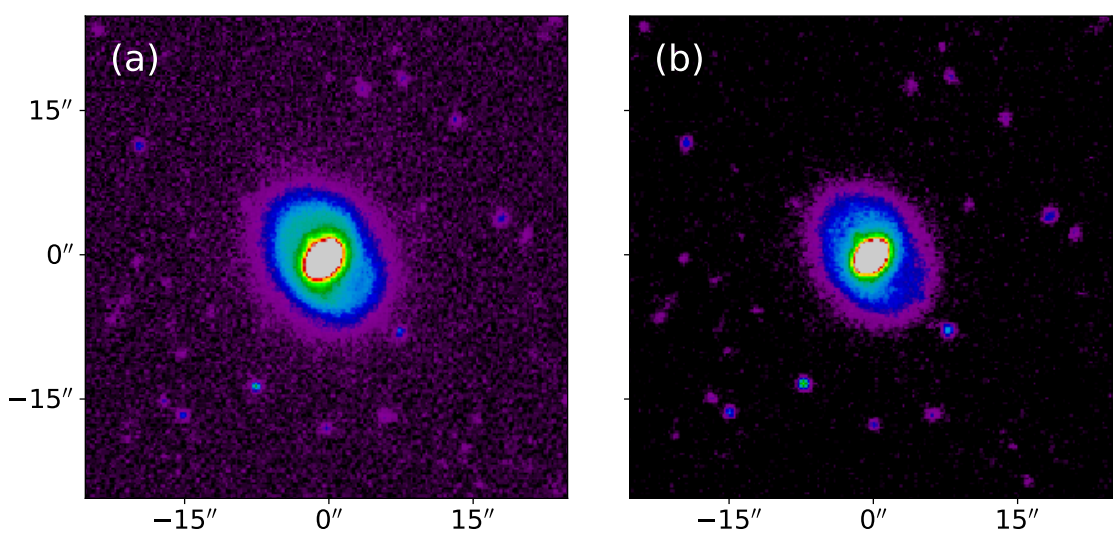

(25) - Gal003114

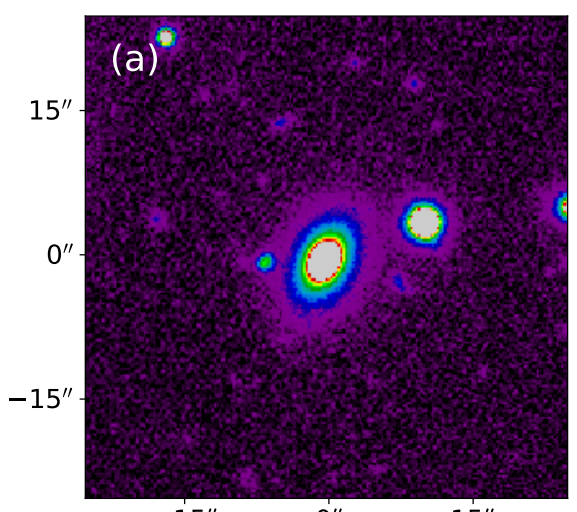

$\begin{array}{lll}-15^{\prime \prime} & 0^{\prime \prime} \quad 15^{\prime \prime}\end{array}$

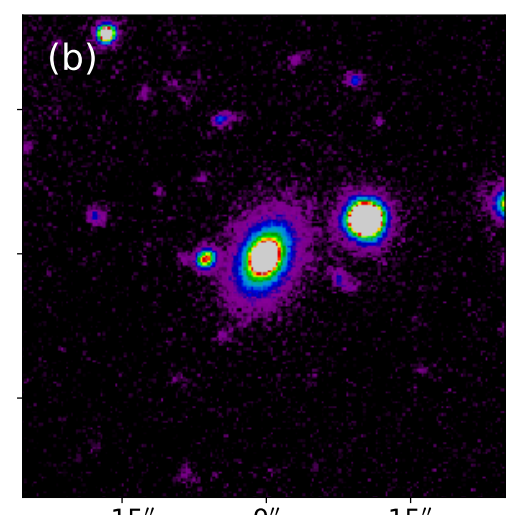

$-15^{\prime \prime}$

$15^{\prime \prime}$

(26) - Gal204260
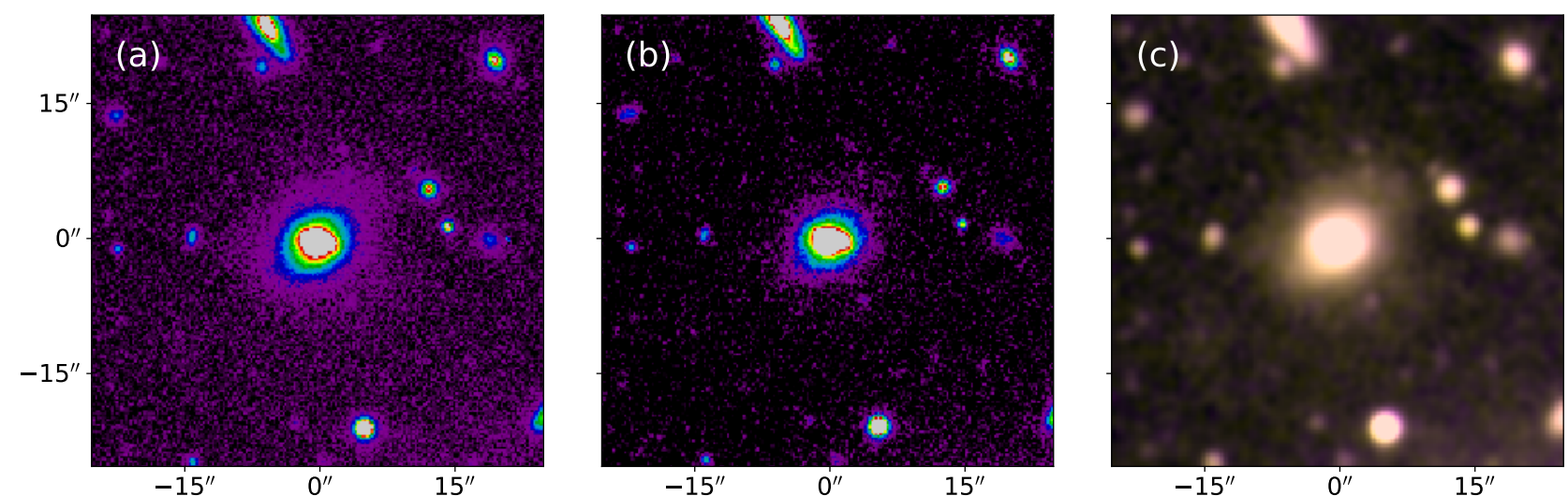

Figure 8. (Continued.)
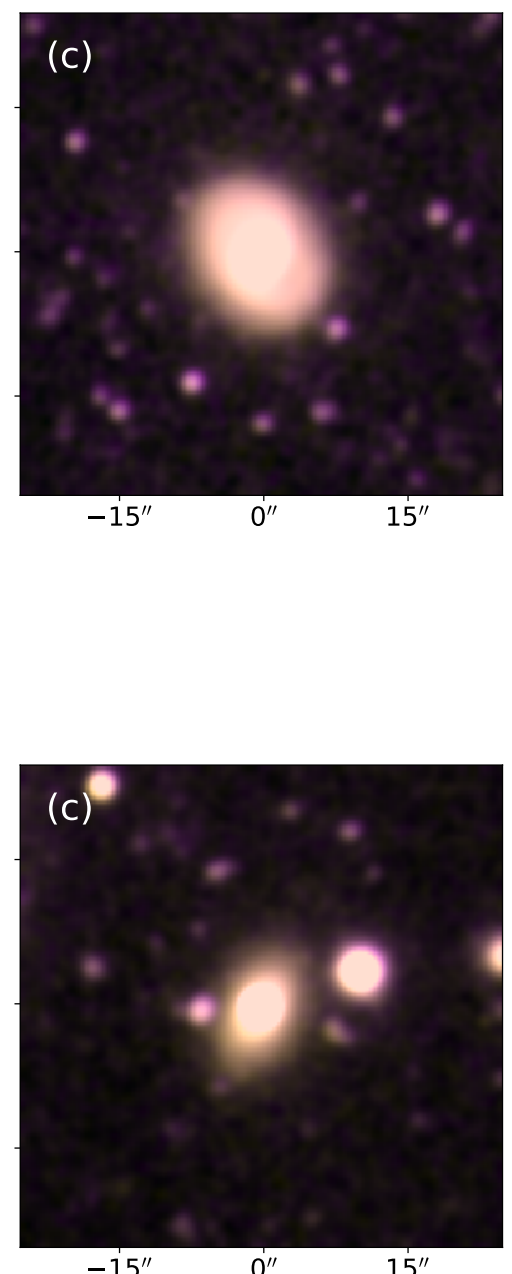

$-15^{\prime \prime}$

$15^{\prime \prime}$ 
(27) - Gal347112
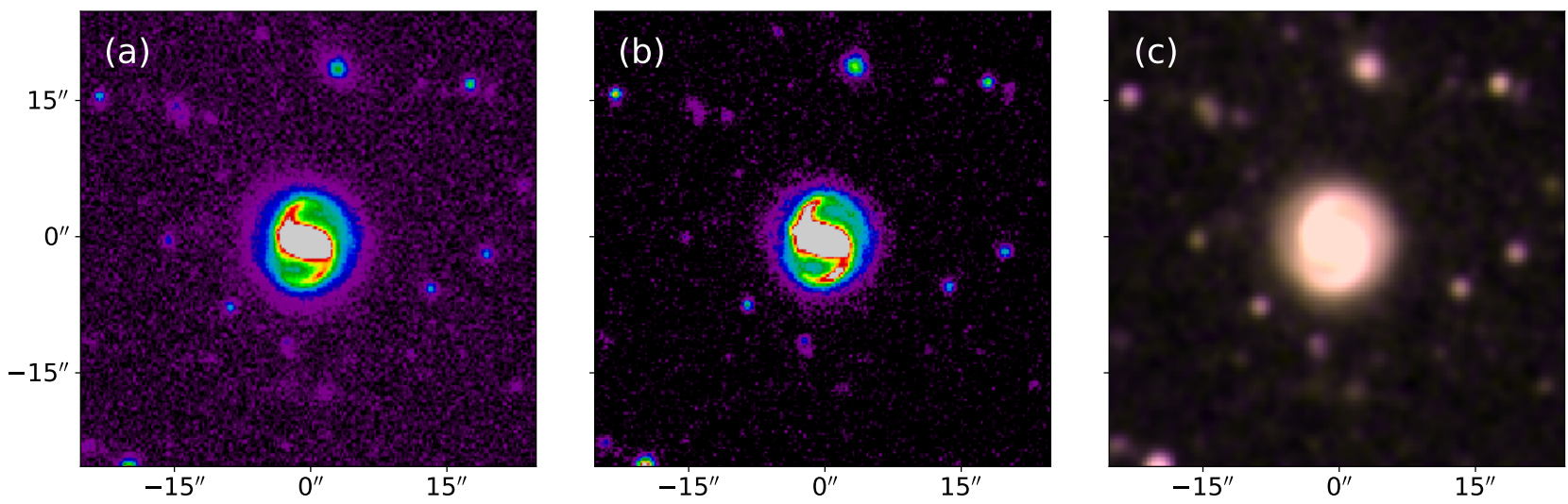

(28) - Gal557614
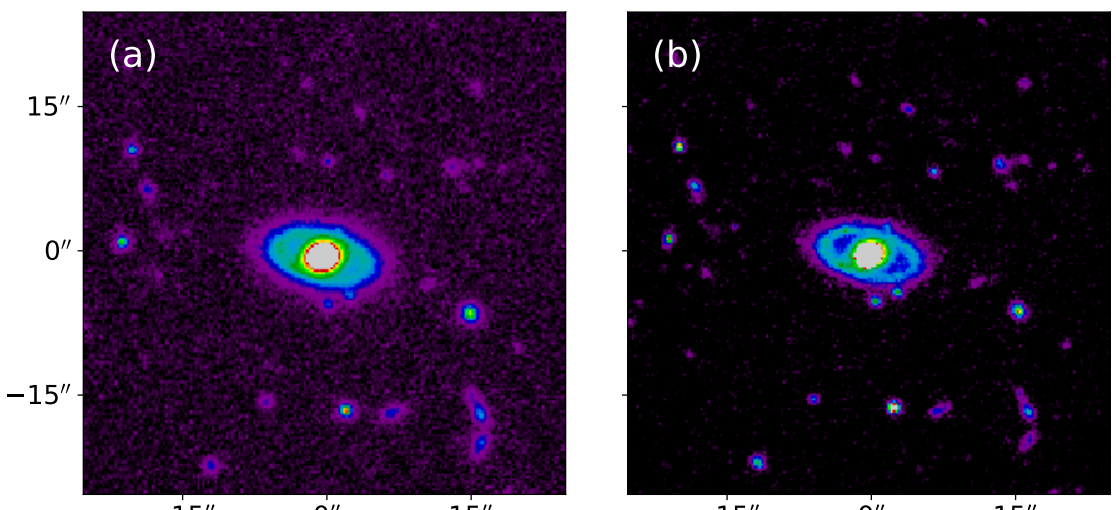

$-15^{\prime \prime}$

$15^{\prime \prime}$

(29) - HE1226+0219
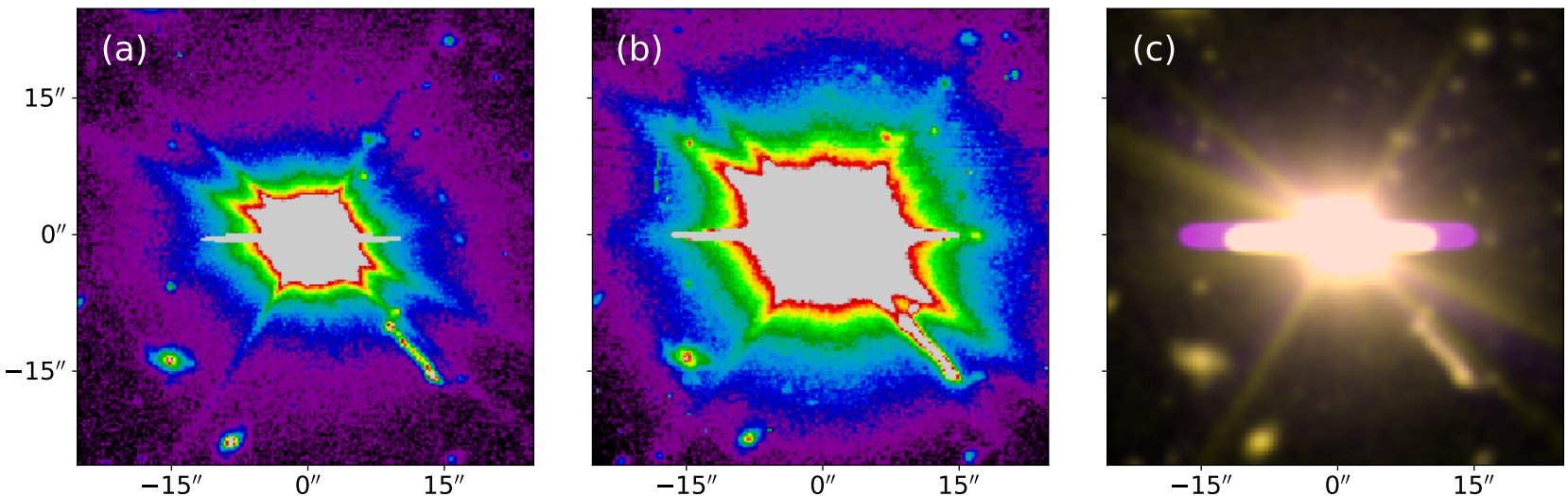

Figure 8. (Continued.)

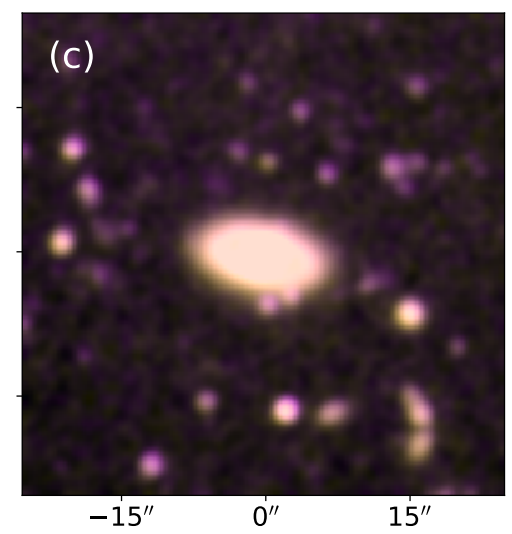


(30) - Gal095873
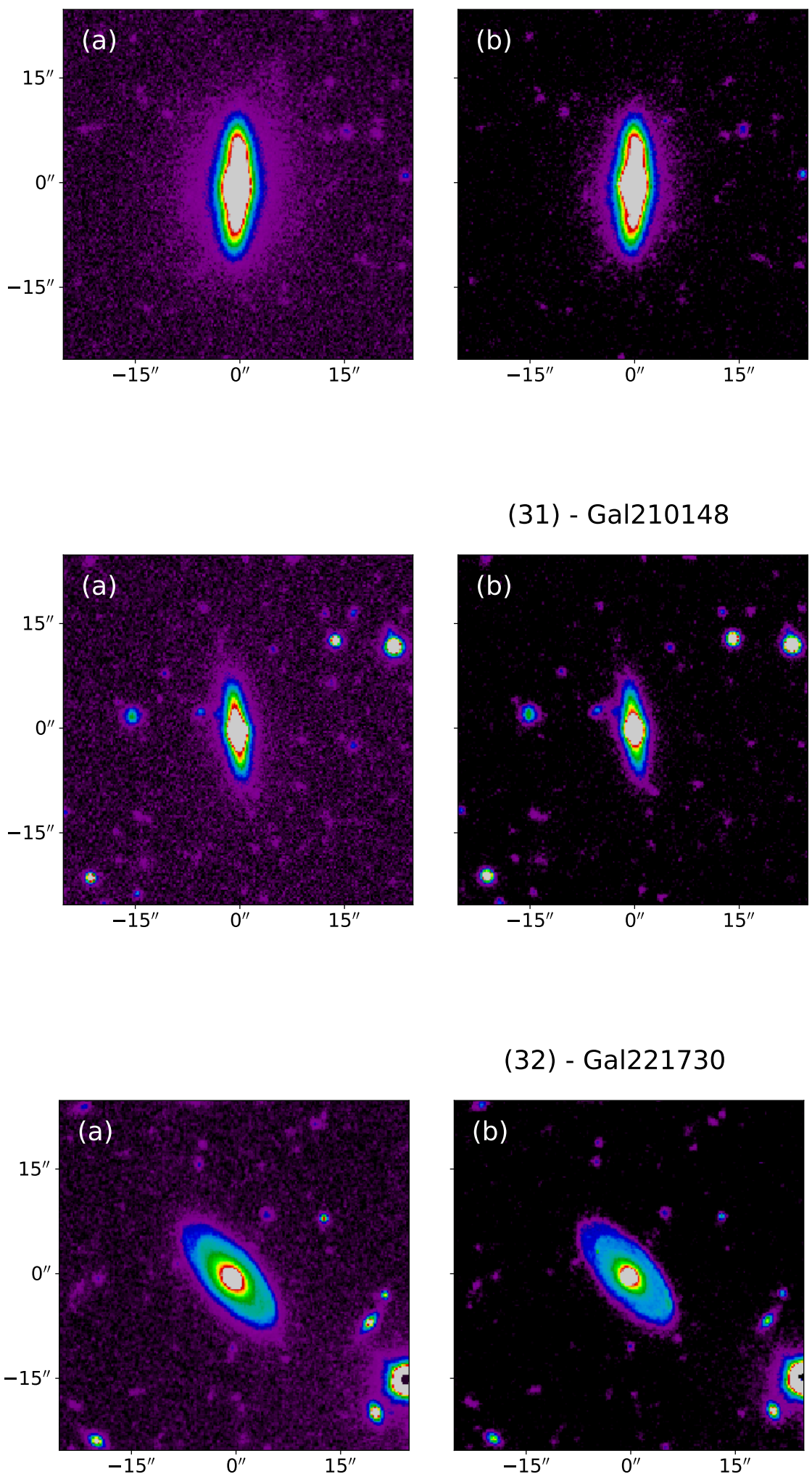

(31) - Gal210148

(32) - Gal221730

(c)
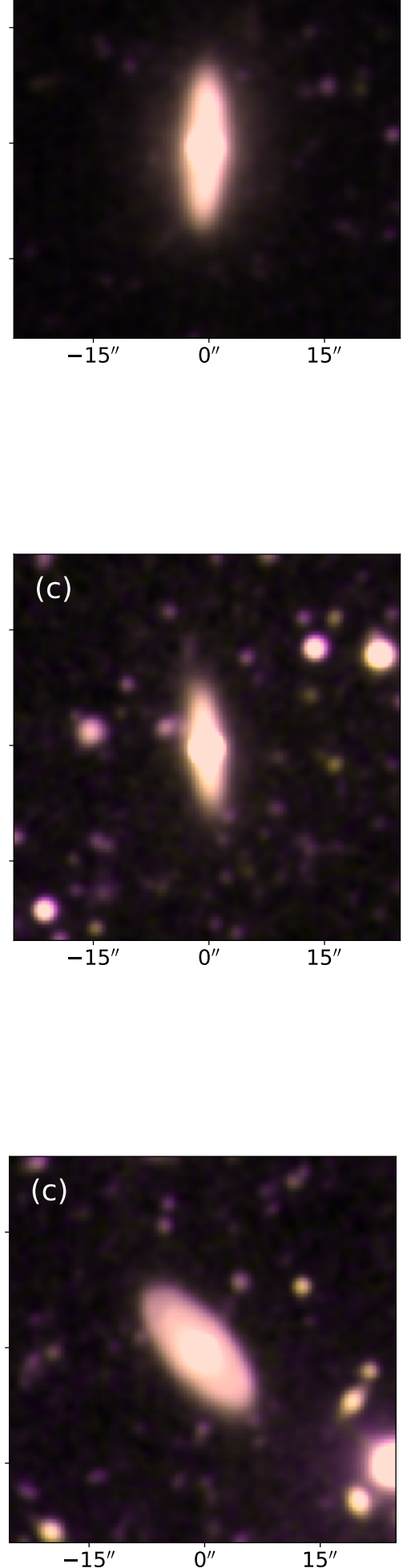

Figure 8. (Continued.) 
(33) - Gal000232
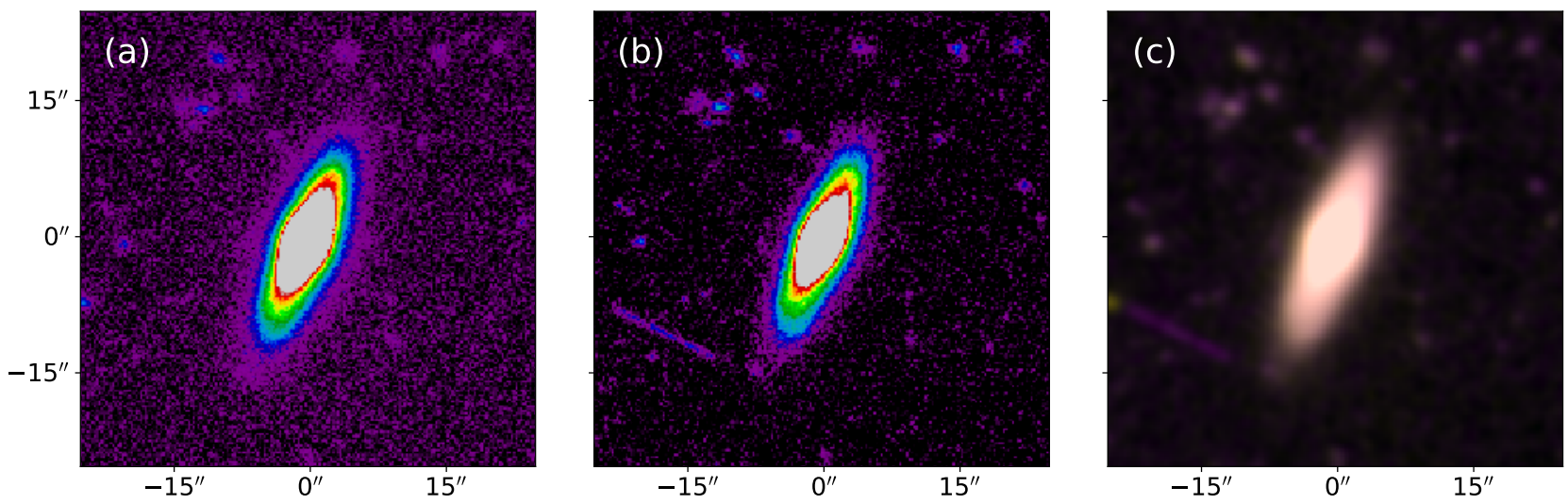

(34) - HE1228+0131
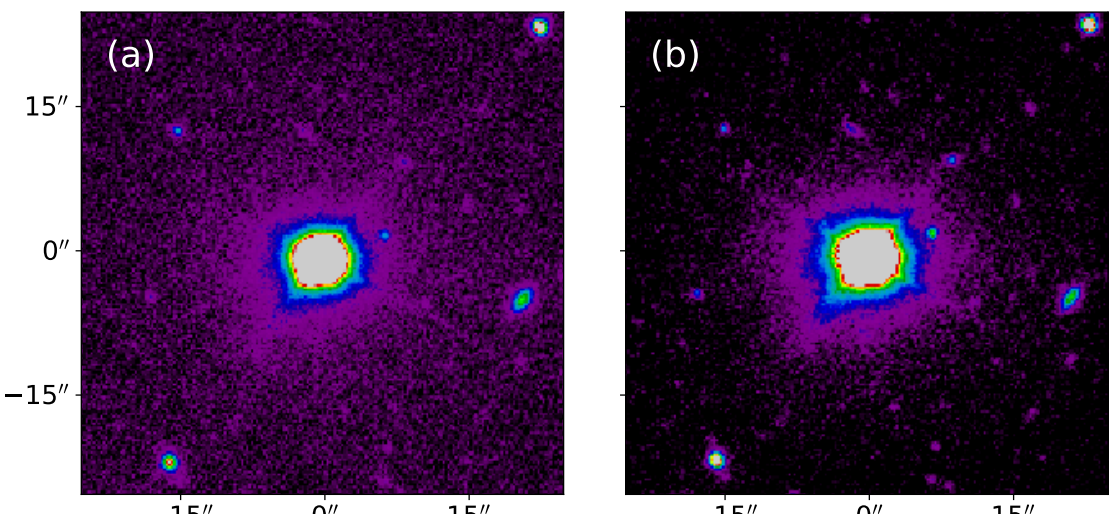

$-15^{\prime \prime}$

$15^{\prime \prime}$

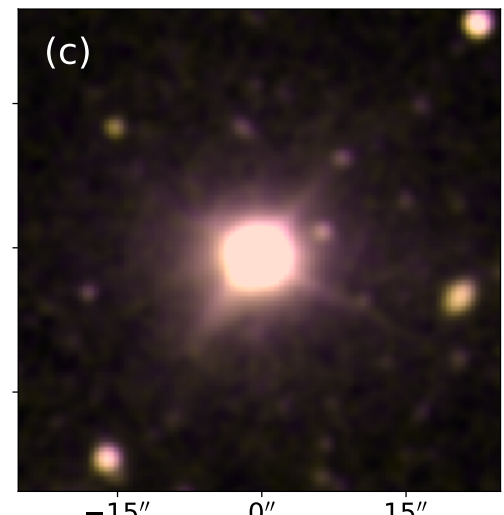

(35) - HE1201-2408
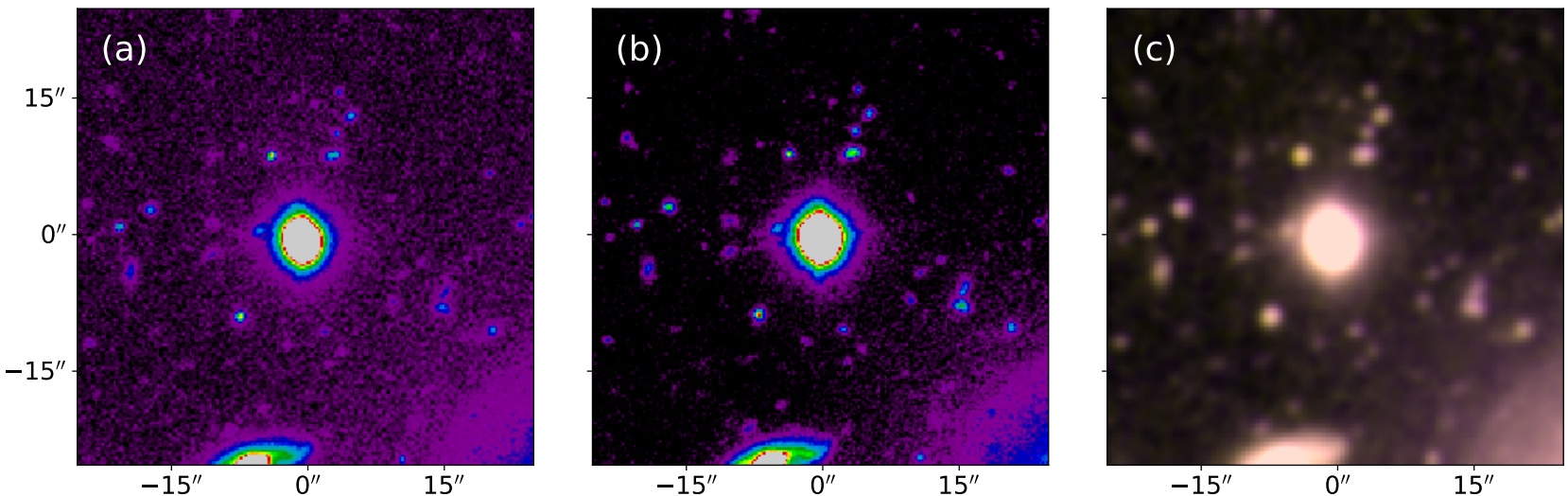

Figure 8. (Continued.) 
(36) - Gal391560
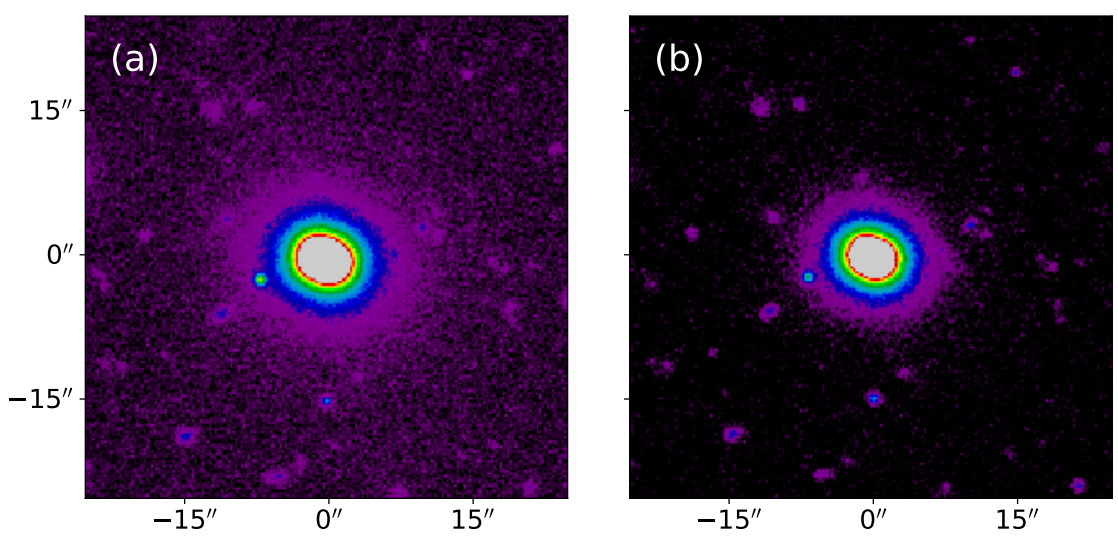

(37) - PG1001+054

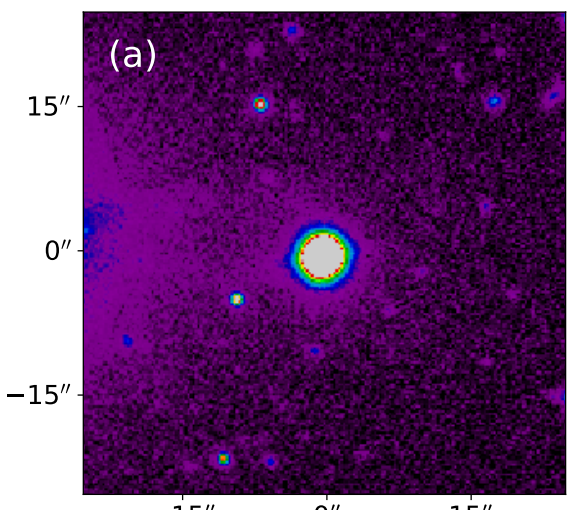

$-15^{\prime \prime}$

$0^{\prime \prime}$

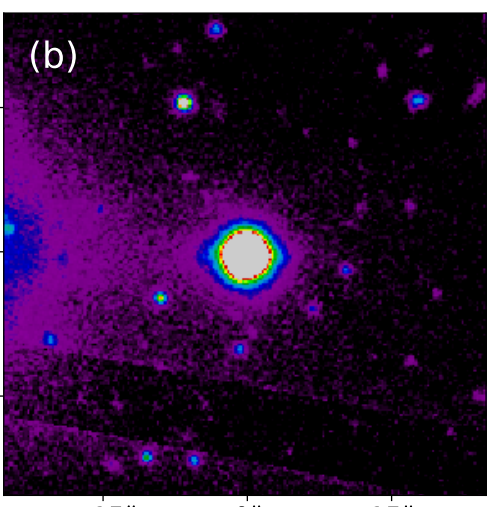

$-15^{\prime \prime}$

$15^{\prime \prime}$

(38) - HE0119-2836

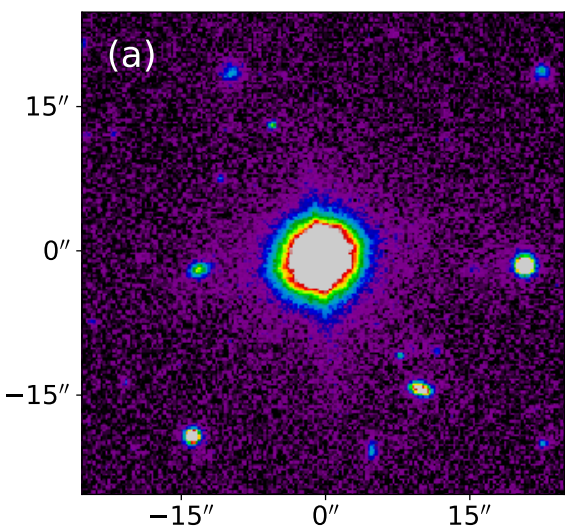

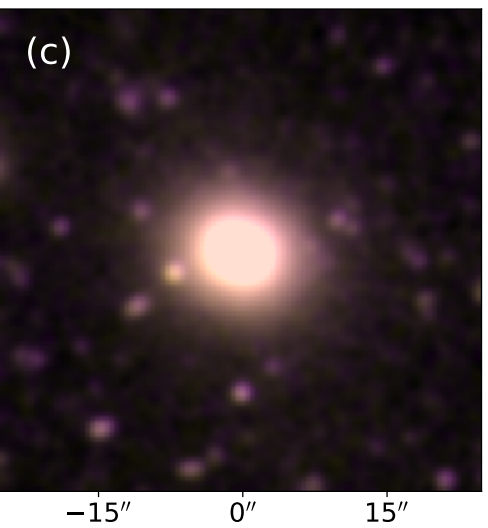
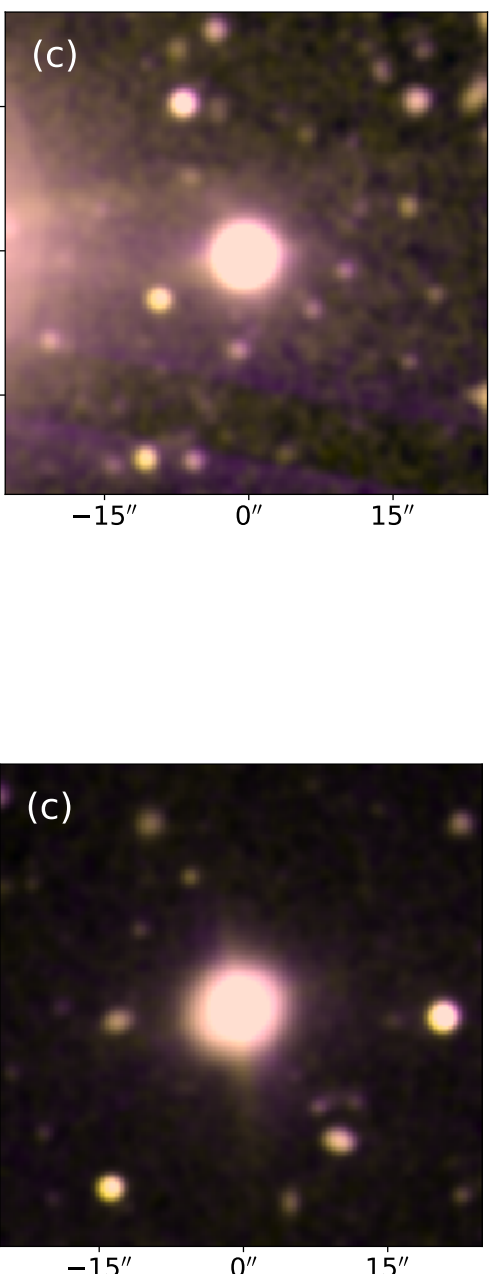

Figure 8. (Continued.) 
(39) - PG1211+143
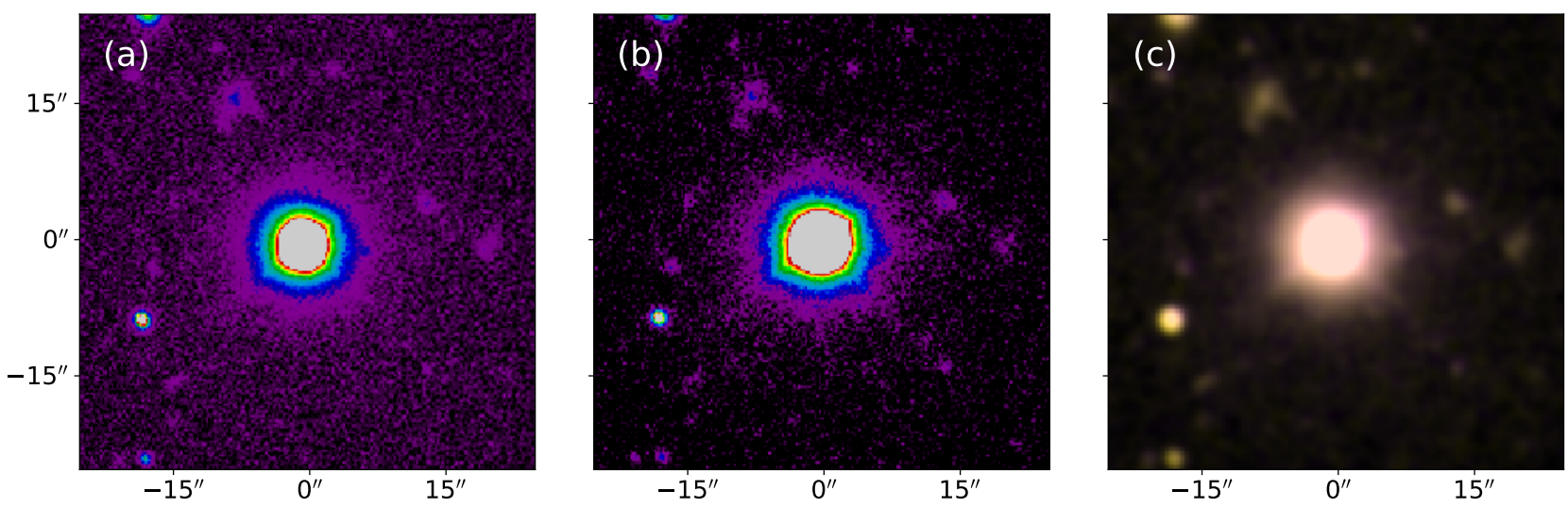

(40) - SDSSJ032213.89+005513.4

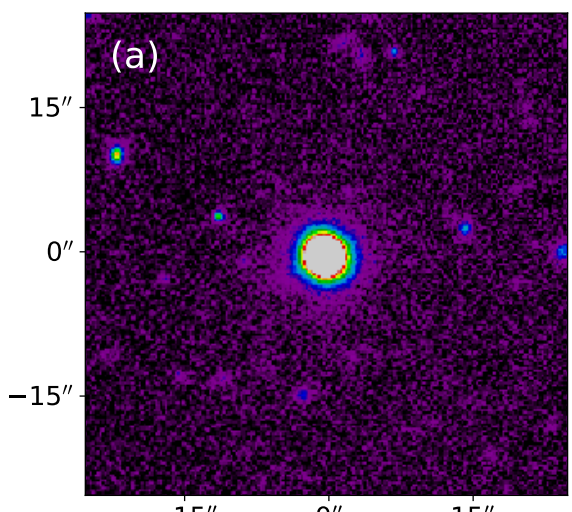

$-15^{\prime \prime}$

$15^{\prime \prime}$

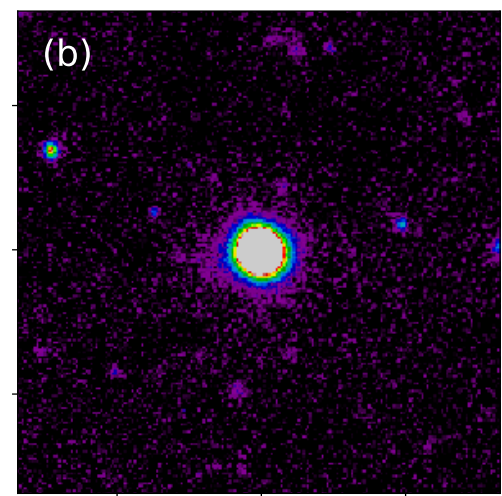

$-15^{\prime \prime}$

$0^{\prime \prime}$

$15^{\prime \prime}$

(41) - Gal656010

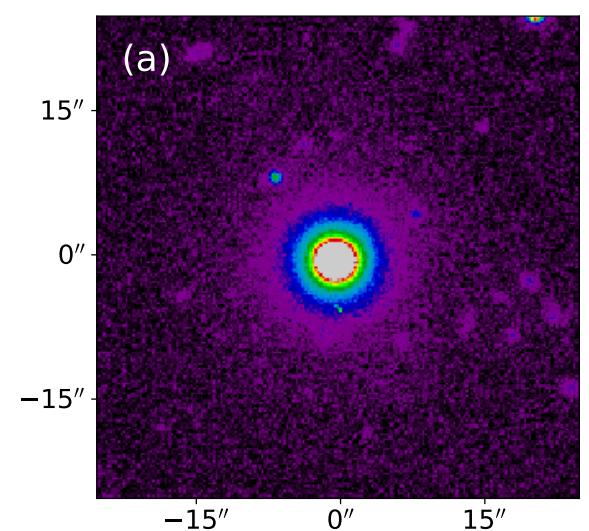

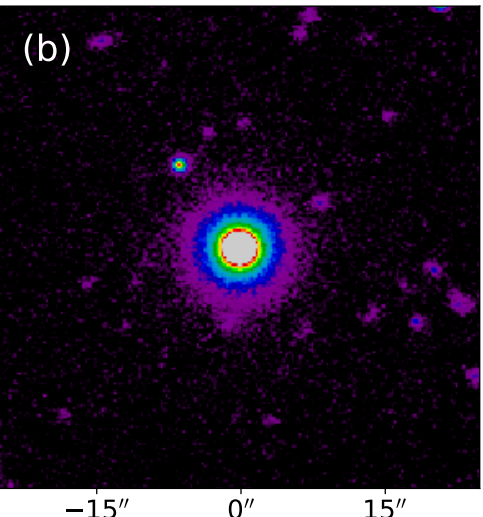

Figure 8. (Continued.)
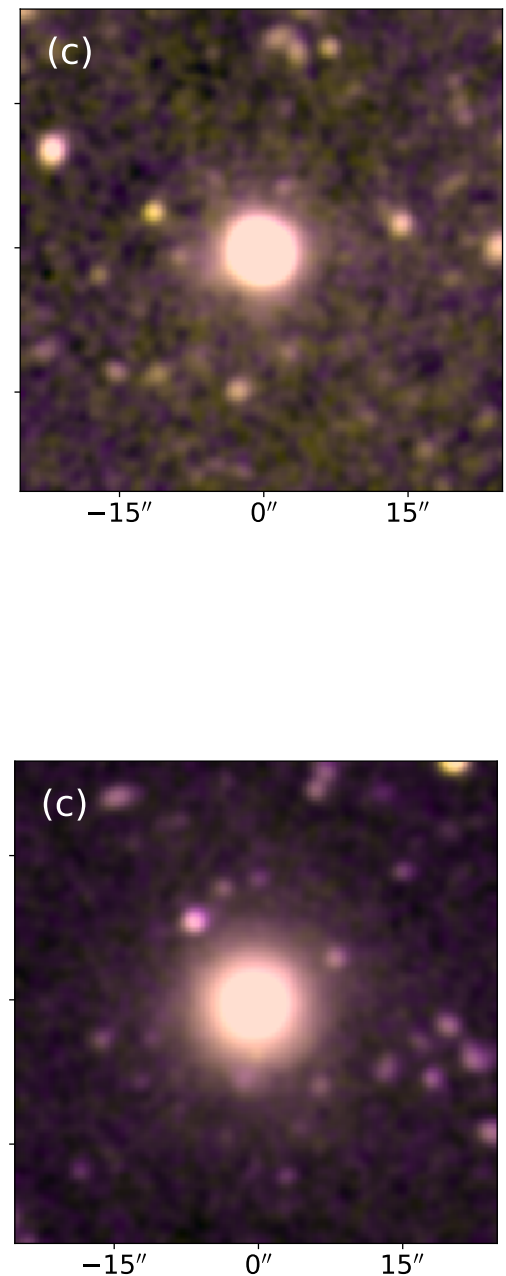

$-15^{\prime \prime}$ 
(42) - Gal176221
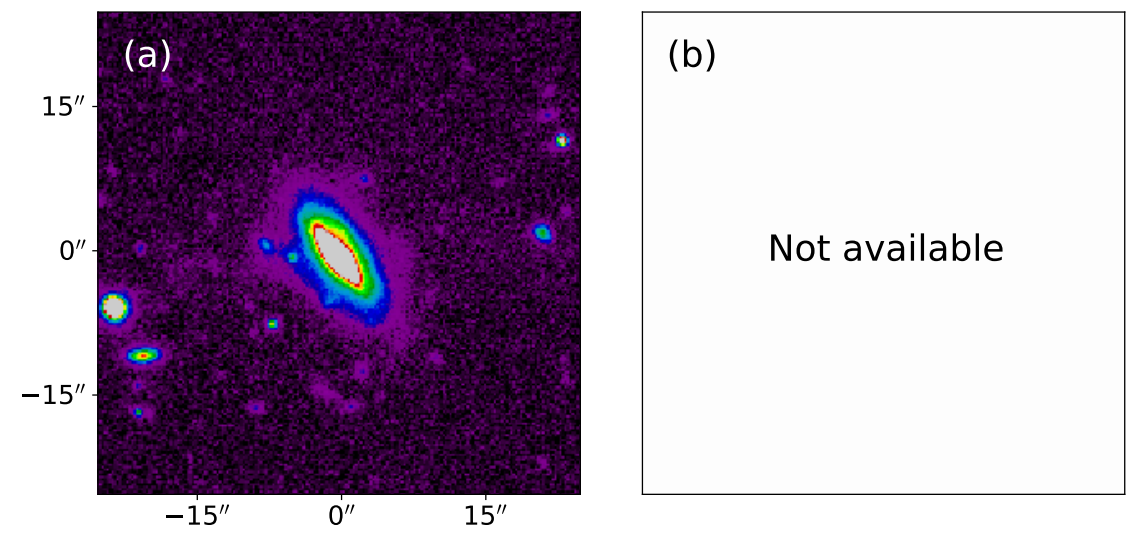

\section{(c)}

Not available

Figure 8. (Continued.)

\section{TABULAR OVERALL CONSENSUS RANKINGS}

Complementary to Appendix $\mathrm{C}$ we present in this section for referential use the consensus ranks for each target for all sets and combination methods. As in Appendix $\mathrm{C}$ the sources are sorted by rank of the 'meta' consensus ranking, i.e. the combined ranking of the nine overall rankings (see Sect. 4.1).

Table 3. Final consensus ranks

\begin{tabular}{|c|c|c|c|c|c|c|c|c|c|}
\hline \multirow[t]{2}{*}{ Target } & \multicolumn{3}{|c|}{ Borda } & \multicolumn{3}{|c|}{ Average } & \multicolumn{3}{|c|}{ Schulze } \\
\hline & V-band & B-band & Color & V-band & B-band & Color & V-band & B-band & Color \\
\hline SDSS-J105007.75+113228.6 & 1 & 3 & 3 & 1 & 4 & 4 & 1 & 3 & 5 \\
\hline Gal030481 & 4 & 6 & 1 & 2 & 5 & 1 & 2 & 5 & 1 \\
\hline HE0157+0009 & 3 & 1 & 4 & 3 & 1 & 6 & 3 & 1 & 6 \\
\hline HE2011-6103 & 2 & 2 & 5 & 4 & 2 & 5 & 4 & 2 & 4 \\
\hline HE2258-5524 & 5 & 4 & 2 & 6 & 6 & 2 & 5 & 4 & 2 \\
\hline HE0132-0441 & 7 & 7 & 6 & 5 & 7 & 3 & 6 & 7 & 3 \\
\hline HE0558-5026 & 6 & 5 & 8 & 7 & 3 & 8 & 7 & 6 & 8 \\
\hline PG1012+008 & 8 & 8 & 7 & 8 & 8 & 7 & 8 & 8 & 7 \\
\hline Gal458007 & 10 & 11 & 9 & 9 & 10 & 9 & 9 & 10 & 10 \\
\hline Gal079769 & 12 & 10 & 10 & 13 & 9 & 13 & 10 & 9 & 9 \\
\hline Gal270096 & 11 & 12 & 12 & 10 & 11 & 12 & 11 & 11 & 11 \\
\hline Gal698144 & 18 & 13 & 13 & 15 & 12 & 10 & 18 & 12 & 12 \\
\hline Gal782980 & 9 & 9 & 15 & 12 & 13 & 16 & 12 & 13 & 16 \\
\hline HE0444-3449 & 13 & 21 & 11 & 11 & 22 & 11 & 13 & 21 & 13 \\
\hline Gal534882 & 15 & 16 & 16 & 20 & 19 & 18 & 17 & 17 & 15 \\
\hline Gal510223 & 19 & 14 & 21 & 17 & 15 & 19 & 22 & 14 & 19 \\
\hline Gal050873 & 20 & 17 & 17 & 19 & 20 & 17 & 19 & 18 & 17 \\
\hline Gal419090 & 22 & 22 & 18 & 18 & 18 & 15 & 20 & 16 & 18 \\
\hline
\end{tabular}

Table 3 continued 
MAJor MERGER RATE For AGNs with The highest EdDington RATios AT $z<0.2$

Table 3 (continued)

\begin{tabular}{|c|c|c|c|c|c|c|c|c|c|}
\hline \multirow[t]{2}{*}{ Target } & \multicolumn{3}{|c|}{ Borda } & \multicolumn{3}{|c|}{ Average } & \multicolumn{3}{|c|}{ Schulze } \\
\hline & V-band & B-band & Color & V-band & B-band & Color & V-band & B-band & Color \\
\hline Gal676011 & 23 & 15 & 20 & 23 & 16 & 21 & 23 & 15 & 20 \\
\hline SDSS-J124341.77+091707.1 & 17 & 19 & 22 & 21 & 14 & 22 & 15 & 22 & 24 \\
\hline Gal498251 & 21 & 18 & 26 & 22 & 17 & 25 & 21 & 19 & 22 \\
\hline Gal286443 & 16 & 20 & 27 & 16 & 21 & 26 & 16 & 20 & 23 \\
\hline HE2152-0936 & 24 & 23 & 37 & 24 & 24 & 35 & 24 & 23 & 35 \\
\hline Gal185580 & 26 & 30 & 14 & 25 & 29 & 14 & 25 & 28 & 14 \\
\hline Gal003114 & 28 & 24 & 29 & 28 & 23 & 27 & 27 & 24 & 26 \\
\hline Gal204260 & 31 & 26 & 19 & 29 & 26 & 20 & 30 & 29 & 21 \\
\hline Gal347112 & 30 & 29 & 23 & 26 & 27 & 23 & 26 & 26 & 27 \\
\hline Gal557614 & 27 & 27 & 24 & 30 & 28 & 24 & 28 & 27 & 25 \\
\hline HE1226+0219 & 25 & 28 & 25 & 27 & 31 & 29 & 33 & 33 & 29 \\
\hline Gal095873 & 29 & 31 & 32 & 31 & 30 & 31 & 29 & 30 & 32 \\
\hline Gal210148 & 33 & 25 & 35 & 33 & 25 & 33 & 31 & 25 & 33 \\
\hline Gal221730 & 34 & 33 & 28 & 32 & 33 & 28 & 35 & 32 & 31 \\
\hline Gal000232 & 36 & 34 & 30 & 37 & 34 & 32 & 34 & 31 & 30 \\
\hline HE1228+0131 & 35 & 32 & 33 & 34 & 32 & 37 & 36 & 34 & 36 \\
\hline HE1201-2408 & 37 & 38 & 34 & 35 & 36 & 34 & 32 & 36 & 34 \\
\hline Gal391560 & 38 & 39 & 31 & 36 & 39 & 30 & 38 & 35 & 28 \\
\hline PG1001+054 & 32 & 36 & 41 & 38 & 35 & 40 & 37 & 37 & 39 \\
\hline HE0119-2836 & 40 & 37 & 39 & 39 & 37 & 38 & 39 & 38 & 38 \\
\hline PG1211+143 & 39 & 35 & 38 & 40 & 38 & 39 & 40 & 39 & 41 \\
\hline SDSS-J032213.89+005513.4 & 41 & 40 & 40 & 41 & 40 & 41 & 42 & 40 & 40 \\
\hline Gal656010 & 42 & 41 & 36 & 42 & 41 & 36 & 41 & 41 & 37 \\
\hline Gal176221 & 14 & $\mathrm{~N} / \mathrm{A}$ & $\mathrm{N} / \mathrm{A}$ & 14 & $\mathrm{~N} / \mathrm{A}$ & $\mathrm{N} / \mathrm{A}$ & 14 & $\mathrm{~N} / \mathrm{A}$ & $\mathrm{N} / \mathrm{A}$ \\
\hline
\end{tabular}

Note - The final ranks for each source depending on combination method (Borda, Average or Schulze) and set $(B, V$ or color images. The targets are sorted by a repeated use of the Schulze method on this nine overall rankings resulting in a singular consensus sequence. Since we have for Gal176221 only observations in $V$-band it is ranked last by the algorithm. 


\section{REFERENCES}

Aird, J., Coil, A. L., \& Georgakakis, A. 2019, Monthly Notices of the Royal Astronomical Society, 484, 4360, doi: 10.1093/mnras/stz125

Aird, J., Coil, A. L., Georgakakis, A., et al. 2015, Monthly Notices of the Royal Astronomical Society, 451, 1892, doi: 10.1093/mnras/stv1062

Allevato, V., Finoguenov, A., Cappelluti, N., et al. 2011, The Astrophysical Journal, 736, 99, doi: 10.1088/0004-637X/736/2/99

Arrow, K. J. 1950, Journal of Political Economy, 58, 328, doi: $10.1086 / 256963$

Astropy Collaboration, Robitaille, T. P., Tollerud, E. J., et al. 2013, \aap, 558, A33, doi: 10.1051/0004-6361/201322068

Astropy Collaboration, Price-Whelan, A. M., Sip \Hocz, B. M., et al. 2018, \aj, 156, 123, doi: 10.3847/1538-3881/aabc4f

Barnes, J. E., \& Hernquist, L. 1992, \araa, 30, 705, doi: 10.1146/annurev.aa.30.090192.003421

Baumgartner, W. H., Tueller, J., Markwardt, C. B., et al. 2013, The Astrophysical Journal Supplement Series, 207, 19, doi: 10.1088/0067-0049/207/2/19

Beifiori, A., Courteau, S., Corsini, E. M., \& Zhu, Y. 2012, Monthly Notices of the Royal Astronomical Society, 419, 2497, doi: 10.1111/j.1365-2966.2011.19903.x

Bennert, V. N., Auger, M. W., Treu, T., Woo, J.-H., \& Malkan, M. A. 2011, The Astrophysical Journal, 742, 107, doi: 10.1088/0004-637X/742/2/107

Bennert, V. N., Treu, T., Woo, J.-H., et al. 2010, The Astrophysical Journal, 708, 1507, doi: 10.1088/0004-637X/708/2/1507

Blanton, E. L., Clarke, T. E., Sarazin, C. L., Randall, S. W., \& McNamara, B. R. 2010, Proceedings of the National Academy of Sciences, 107, 7174, doi: 10.1073/pnas.0913904107

Bottrell, C., Hani, M. H., Teimoorinia, H., et al. 2019, Monthly Notices of the Royal Astronomical Society, 490, 5390, doi: 10.1093/mnras/stz2934

Boyle, B. J., Shanks, T., Croom, S. M., et al. 2000, Monthly Notices of the Royal Astronomical Society, 317, 1014, doi: 10.1046/j.1365-8711.2000.03730.x

Bradley, L., Sipcz, B., Robitaille, T., et al. 2019, astropy/photutils: v0.7.2, Zenodo, doi: 10.5281 /zenodo. 3568287

Bridge, C. R., Carlberg, R. G., \& Sullivan, M. 2010, The Astrophysical Journal, 709, 1067, doi: 10.1088/0004-637X/709/2/1067
Brinchmann, J., Charlot, S., White, S. D. M., et al. 2004, Monthly Notices of the Royal Astronomical Society, 351, 1151, doi: 10.1111/j.1365-2966.2004.07881.x

Bhm, A., Wisotzki, L., Bell, E. F., et al. 2013, Astronomy \& Astrophysics, 549, A46, doi: 10.1051/0004-6361/201015444

Casteels, K. R. V., Conselice, C. J., Bamford, S. P., et al. 2014, Monthly Notices of the Royal Astronomical Society, 445, 1157, doi: 10.1093/mnras/stu1799

Cen, R., \& Safarzadeh, M. 2015, The Astrophysical Journal Letters, 798, L38, doi: 10.1088/2041-8205/798/2/L38

Cheng, T.-Y., Conselice, C. J., Aragn-Salamanca, A., et al. 2019, arXiv e-prints, 1908, arXiv:1908.03610. http://adsabs.harvard.edu/abs/2019arXiv190803610C

Cheung, E., Trump, J. R., Athanassoula, E., et al. 2015, Monthly Notices of the Royal Astronomical Society, 447, 506, doi: 10.1093/mnras/stu2462

Chowdhury, R. K., Chatterjee, S., Lonappan, A. I., Khandai, N., \& DiMatteo, T. 2019, arXiv:1911.07824 [astro-ph]. http://arxiv.org/abs/1911.07824

Churazov, E., Sazonov, S., Sunyaev, R., et al. 2005, Monthly Notices of the Royal Astronomical Society: Letters, 363, L91, doi: 10.1111/j.1745-3933.2005.00093.x

Cigan, P. 2019, MultiColorFits: Colorize and combine multiple fits images for visually aesthetic scientific plots. https://ascl.net/1909.002

Cisternas, M., Sheth, K., Salvato, M., et al. 2015, The Astrophysical Journal, 802, 137, doi: 10.1088/0004-637X/802/2/137

Cisternas, M., Jahnke, K., Inskip, K. J., et al. 2011, The Astrophysical Journal, 726, 57, doi: 10.1088/0004-637X/726/2/57

Condorcet, J.-A.-N. d. C. .-. . m. d. A. d. t. 1785, Essai sur l'application de l'analyse la probabilit des dcisions rendues la pluralit des voix ([Reprod.]) / par M. le marquis de Condorcet,... (de l'Impr. royale (Paris)). https://gallica.bnf.fr/ark:/12148/bpt6k417181

Condorcet, J.-A.-N. d. C., Sommerlad, F., McLean, I., University of Oxford, \& Faculty of Social Studies. 1989, The political theory of Condorcet (Oxford: University of Oxford, Faculty of Social Studies)

Conroy, C., \& White, M. 2013, The Astrophysical Journal, 762, 70, doi: 10.1088/0004-637X/762/2/70

Cotini, S., Ripamonti, E., Caccianiga, A., et al. 2013, Monthly Notices of the Royal Astronomical Society, 431, 2661, doi: 10.1093/mnras/stt358

Dashyan, G., Choi, E., Somerville, R. S., et al. 2019, Monthly Notices of the Royal Astronomical Society, 487, 5889, doi: 10.1093/mnras/stz1697 
Davies, J. J., Crain, R. A., Oppenheimer, B. D., \& Schaye, J. 2019, arXiv e-prints, 1908, arXiv:1908.11380.

http://adsabs.harvard.edu/abs/2019arXiv190811380D

Davis, B. L., Graham, A. W., \& Cameron, E. 2018, The Astrophysical Journal, 869, 113, doi: 10.3847/1538-4357/aae820

- 2019, The Astrophysical Journal, 873, 85, doi: 10.3847/1538-4357/aaf3b8

de Nicola, S., Marconi, A., \& Longo, G. 2019, Monthly Notices of the Royal Astronomical Society, 490, 600, doi: $10.1093 / \mathrm{mnras} / \mathrm{stz} 2472$

Delvecchio, I., Lutz, D., Berta, S., et al. 2015, Monthly Notices of the Royal Astronomical Society, 449, 373, doi: 10.1093/mnras/stv213

Di Matteo, T., Springel, V., \& Hernquist, L. 2005, Nature, 433, 604, doi: 10.1038/nature03335

Ding, X., Silverman, J., Treu, T., et al. 2020, The Astrophysical Journal, 888, 37, doi: 10.3847/1538-4357/ab5b90

Donley, J. L., Kartaltepe, J., Kocevski, D., et al. 2018, The Astrophysical Journal, 853, 63, doi: 10.3847/1538-4357/aa9ffa

Duncan, K., Conselice, C. J., Mundy, C., et al. 2019, The Astrophysical Journal, 876, 110, doi: $10.3847 / 1538-4357 /$ ab148a

Ellison, S. L., Viswanathan, A., Patton, D. R., et al. 2019, MNRAS, 487, 2491, doi: 10.1093/mnras/stz1431

Emerson, P. 2013, Social Choice and Welfare, 40, 353, doi: 10.1007/s00355-011-0603-9

Erben, T., Schirmer, M., Dietrich, J. P., et al. 2005, Astronomische Nachrichten, 326, 432, doi: 10.1002/asna.200510396

Fan, L., Han, Y., Fang, G., et al. 2016, The Astrophysical Journal, 822, L32, doi: 10.3847/2041-8205/822/2/L32

Fanidakis, N., Baugh, C. M., Benson, A. J., et al. 2012, Monthly Notices of the Royal Astronomical Society, 419, 2797, doi: 10.1111/j.1365-2966.2011.19931.x

Furlong, M., Bower, R. G., Theuns, T., et al. 2015, Monthly Notices of the Royal Astronomical Society, 450, 4486, doi: 10.1093/mnras/stv852

Gabor, J. M., Impey, C. D., Jahnke, K., et al. 2009, The Astrophysical Journal, 691, 705, doi: 10.1088/0004-637X/691/1/705

Gao, F., Wang, L., Pearson, W. J., et al. 2020, arXiv:2004.00680 [astro-ph]. http://arxiv.org/abs/2004.00680

Georgakakis, A., Coil, A. L., Laird, E. S., et al. 2009, Monthly Notices of the Royal Astronomical Society, 397, 623, doi: 10.1111/j.1365-2966.2009.14951.x
Glikman, E., Simmons, B., Mailly, M., et al. 2015, The Astrophysical Journal, 806, 218, doi: 10.1088/0004-637X/806/2/218

Goulding, A. D., Matthaey, E., Greene, J. E., et al. 2017, The Astrophysical Journal, 843, 135, doi: 10.3847/1538-4357/aa755b

Goulding, A. D., Greene, J. E., Bezanson, R., et al. 2018, Publications of the Astronomical Society of Japan, 70, doi: $10.1093 / \mathrm{pasj} / \mathrm{psx} 135$

Graham, A. W., \& Scott, N. 2013, The Astrophysical Journal, 764, 151, doi: 10.1088/0004-637X/764/2/151

Grogin, N. A., Conselice, C. J., Chatzichristou, E., et al. 2005, The Astrophysical Journal, 627, L97, doi: $10.1086 / 432256$

Grogin, N. A., Kocevski, D. D., Faber, S. M., et al. 2011, The Astrophysical Journal Supplement Series, 197, 35, doi: 10.1088/0067-0049/197/2/35

Habouzit, M., Genel, S., Somerville, R. S., et al. 2019, Monthly Notices of the Royal Astronomical Society, 484, 4413, doi: 10.1093/mnras/stz102

Harrison, C. M., Costa, T., Tadhunter, C. N., et al. 2018, Nature Astronomy, 2, 198, doi: 10.1038/s41550-018-0403-6

Henriques, B. M. B., White, S. D. M., Thomas, P. A., et al. 2015, Monthly Notices of the Royal Astronomical Society, 451, 2663, doi: 10.1093/mnras/stv705

Hewlett, T., Villforth, C., Wild, V., et al. 2017, Monthly Notices of the Royal Astronomical Society, 470, 755, doi: 10.1093/mnras/stx997

Hirschmann, M., Dolag, K., Saro, A., et al. 2014, Monthly Notices of the Royal Astronomical Society, 442, 2304, doi: 10.1093/mnras/stu1023

Hong, J., Im, M., Kim, M., \& Ho, L. C. 2015, The Astrophysical Journal, 804, 34, doi: 10.1088/0004-637X/804/1/34

Hopkins, P. F., \& Hernquist, L. 2009, The Astrophysical Journal, 698, 1550, doi: 10.1088/0004-637X/698/2/1550

Hopkins, P. F., Hernquist, L., Cox, T. J., et al. 2006a, The Astrophysical Journal Supplement Series, 163, 1, doi: 10.1086/499298

Hopkins, P. F., Hernquist, L., Cox, T. J., \& Kere, D. 2008, The Astrophysical Journal Supplement Series, 175, 356, doi: $10.1086 / 524362$

Hopkins, P. F., Hernquist, L., Martini, P., et al. 2005, The Astrophysical Journal, 625, L71, doi: 10.1086/431146

Hopkins, P. F., \& Quataert, E. 2010, Monthly Notices of the Royal Astronomical Society, 407, 1529, doi: 10.1111/j.1365-2966.2010.17064.x

Hopkins, P. F., Richards, G. T., \& Hernquist, L. 2007, The Astrophysical Journal, 654, 731, doi: 10.1086/509629 
Hopkins, P. F., Somerville, R. S., Hernquist, L., et al. 2006b, The Astrophysical Journal, 652, 864, doi: 10.1086/508503

Hunter, J. D. 2007, Computing in Science and Engineering, 9, 90, doi: 10.1109/MCSE.2007.55

Husemann, B., \& Harrison, C. M. 2018, Nature Astronomy, 2, 196, doi: 10.1038/s41550-018-0407-2

Hring, N., \& Rix, H.-W. 2004, The Astrophysical Journal Letters, 604, L89, doi: 10.1086/383567

Jahnke, K., \& Macci, A. V. 2011, The Astrophysical Journal, 734, 92, doi: 10.1088/0004-637X/734/2/92

Jahnke, K., Bongiorno, A., Brusa, M., et al. 2009, The Astrophysical Journal, 706, L215, doi: 10.1088/0004-637X/706/2/L215

Johansson, P. H., Burkert, A., \& Naab, T. 2009a, The Astrophysical Journal, 707, L184, doi: 10.1088/0004-637X/707/2/L184

Johansson, P. H., Naab, T., \& Burkert, A. 2009b, The Astrophysical Journal, 690, 802, doi: 10.1088/0004-637X/690/1/802

Joye, W. A., \& Mandel, E. 2003, Astronomical Society of the Pacific Conference Series, 295, 489.

http://adsabs.harvard.edu/abs/2003ASPC..295..489J

Jung, M., Illenseer, T. F., \& Duschl, W. J. 2018, Astronomy \& Astrophysics, 614, A105, doi: 10.1051/0004-6361/201731688

Karouzos, M., Jarvis, M. J., \& Bonfield, D. 2014, Monthly Notices of the Royal Astronomical Society, 439, 861, doi: 10.1093/mnras/stu009

Kauffmann, G., Heckman, T. M., White, S. D. M., et al. 2003, Monthly Notices of the Royal Astronomical Society, 341, 33, doi: 10.1046/j.1365-8711.2003.06291.x

Kocevski, D. D., Faber, S. M., Mozena, M., et al. 2012, The Astrophysical Journal, 744, 148, doi: 10.1088/0004-637X/744/2/148

Koekemoer, A. M., Faber, S. M., Ferguson, H. C., et al. 2011, The Astrophysical Journal Supplement Series, 197, 36, doi: 10.1088/0067-0049/197/2/36

Kormendy, J., \& Ho, L. C. 2013, Annual Review of Astronomy and Astrophysics, 51, 511, doi: 10.1146/annurev-astro-082708-101811

Koss, M., Mushotzky, R., Veilleux, S., \& Winter, L. 2010, The Astrophysical Journal, 716, L125, doi: 10.1088/2041-8205/716/2/L125

Koss, M. J., Blecha, L., Bernhard, P., et al. 2018, Nature, 563, 214, doi: 10.1038/s41586-018-0652-7

Lacey, C. G., Baugh, C. M., Frenk, C. S., et al. 2016, Monthly Notices of the Royal Astronomical Society, 462, 3854, doi: 10.1093/mnras/stw1888
Li, Y., Habouzit, M., Genel, S., et al. 2019a, arXiv:1910.00017 [astro-ph]. http://arxiv.org/abs/1910.00017

Li, Y., Gendron-Marsolais, M.-L., Zhuravleva, I., et al. 2019b, arXiv:1911.06329 [astro-ph]. http://arxiv.org/abs/1911.06329

Lotz, J. M., Jonsson, P., Cox, T. J., et al. 2011, The Astrophysical Journal, 742, 103, doi: 10.1088/0004-637X/742/2/103

Lotz, J. M., Jonsson, P., Cox, T. J., \& Primack, J. R. 2008a, Monthly Notices of the Royal Astronomical Society, 391, 1137, doi: 10.1111/j.1365-2966.2008.14004.x

Lotz, J. M., Davis, M., Faber, S. M., et al. 2008b, The Astrophysical Journal, 672, 177, doi: 10.1086/523659

Lpez-Sanjuan, C., Balcells, M., Prez-Gonzlez, P. G., et al. 2009, Astronomy \& Astrophysics, 501, 505, doi: 10.1051/0004-6361/200911923

Madau, P., \& Dickinson, M. 2014, Annual Review of Astronomy and Astrophysics, 52, 415, doi: 10.1146/annurev-astro-081811-125615

Man, A. W. S., Zirm, A. W., \& Toft, S. 2016, The Astrophysical Journal, 830, 89, doi: 10.3847/0004-637X/830/2/89

Man, Z.-y., Peng, Y.-j., Kong, X., et al. 2019, Monthly Notices of the Royal Astronomical Society, 488, 89, doi: $10.1093 / \mathrm{mnras} / \mathrm{stz} 1706$

Marconi, A., \& Hunt, L. K. 2003, The Astrophysical Journal, 589, L21, doi: 10.1086/375804

Marian, V., Jahnke, K., Mechtley, M., et al. 2019, The Astrophysical Journal, 882, 141, doi: 10.3847/1538-4357/ab385b

Martin-Navarro, I., Burchett, J. N., \& Mezcua, M. 2019, The Astrophysical Journal, 884, L45, doi: 10.3847/2041-8213/ab4885

Martini, P. 2004, Coevolution of Black Holes and Galaxies, 169.

http://adsabs.harvard.edu/abs/2004cbhg.symp..169M

McAlpine, S., Bower, R. G., Rosario, D. J., et al. 2018, Monthly Notices of the Royal Astronomical Society, 481, 3118, doi: 10.1093/mnras/sty2489

McAlpine, S., Harrison, C. M., Rosario, D. J., et al. 2020, arXiv:2002.00959 [astro-ph].

http://arxiv.org/abs/2002.00959

McConnell, N. J., \& Ma, C.-P. 2013, The Astrophysical Journal, 764, 184, doi: 10.1088/0004-637X/764/2/184

Mechtley, M., Jahnke, K., Windhorst, R. A., et al. 2016, The Astrophysical Journal, 830, 156, doi: 10.3847/0004-637X/830/2/156 
Mundy, C. J., Conselice, C. J., Duncan, K. J., et al. 2017, Monthly Notices of the Royal Astronomical Society, 470, 3507, doi: 10.1093/mnras/stx1238

Nelson, D., Pillepich, A., Springel, V., et al. 2019, Monthly Notices of the Royal Astronomical Society, 490, 3234, doi: 10.1093/mnras/stz2306

Netzer, H. 2019, Monthly Notices of the Royal Astronomical Society, 488, 5185, doi: 10.1093/mnras/stz2016

O'Leary, J. A., Moster, B. P., Naab, T., \& Somerville, R. S. 2020, arXiv:2001.02687 [astro-ph].

http://arxiv.org/abs/2001.02687

Oppenheimer, B. D., Davies, J. J., Crain, R. A., et al. 2020, Monthly Notices of the Royal Astronomical Society, 491, 2939, doi: 10.1093/mnras/stz3124

Pillepich, A., Springel, V., Nelson, D., et al. 2018, Monthly Notices of the Royal Astronomical Society, 473, 4077, doi: 10.1093/mnras/stx2656

Popping, G., Caputi, K. I., Trager, S. C., et al. 2015, Monthly Notices of the Royal Astronomical Society, 454, 2258, doi: 10.1093/mnras/stv2136

Reilly, B. 2002, International Political Science Review, 23, 355, doi: 10.1177/0192512102023004002

Ricarte, A., Tremmel, M., Natarajan, P., \& Quinn, T. 2019, Monthly Notices of the Royal Astronomical Society, 489, 802, doi: 10.1093/mnras/stz2161

Rosario, D. J., McIntosh, D. H., van der Wel, A., et al. 2015, Astronomy \& Astrophysics, 573, A85, doi: 10.1051/0004-6361/201423782

Sabater, J., Best, P. N., \& Argudo-Fernndez, M. 2013, Monthly Notices of the Royal Astronomical Society, 430, 638, doi: 10.1093/mnras/sts675

Sahu, N., Graham, A. W., \& Davis, B. L. 2019, The Astrophysical Journal, 887, 10, doi: 10.3847/1538-4357/ab50b7

Sanders, D. B., \& Mirabel, I. F. 1996, Annual Review of Astronomy and Astrophysics, 34, 749, doi: 10.1146/annurev.astro.34.1.749

Sanders, D. B., Soifer, B. T., Elias, J. H., et al. 1988, The Astrophysical Journal, 325, 74, doi: 10.1086/165983

Santini, P., Maiolino, R., Magnelli, B., et al. 2014, Astronomy \& Astrophysics, 562, A30, doi: 10.1051/0004-6361/201322835

Schawinski, K., Simmons, B. D., Urry, C. M., Treister, E., \& Glikman, E. 2012, Monthly Notices of the Royal Astronomical Society: Letters, 425, L61, doi: 10.1111/j.1745-3933.2012.01302.x

Schawinski, K., Treister, E., Urry, C. M., et al. 2011, The Astrophysical Journal, 727, L31, doi: 10.1088/2041-8205/727/2/L31
Schirmer, M. 2013, The Astrophysical Journal Supplement Series, 209, 21, doi: 10.1088/0067-0049/209/2/21

Schulze, A., \& Wisotzki, L. 2010, Astronomy and Astrophysics, 516, A87, doi: 10.1051/0004-6361/201014193

Schulze, A., Bongiorno, A., Gavignaud, I., et al. 2015, Monthly Notices of the Royal Astronomical Society, 447, 2085, doi: 10.1093/mnras/stu2549

Schulze, A., Silverman, J. D., Daddi, E., et al. 2019, Monthly Notices of the Royal Astronomical Society, 488, 1180, doi: 10.1093/mnras/stz1746

Schulze, M. 2011, Social Choice and Welfare, 36, 267, doi: 10.1007/s00355-010-0475-4

—. 2018, arXiv e-prints, 368. https://arxiv.org/abs/1804.02973

Shankar, F., Weinberg, D. H., Marsden, C., et al. 2019, arXiv:1912.06153 [astro-ph].

http://arxiv.org/abs/1912.06153

Shen, Y., Strauss, M. A., Oguri, M., et al. 2007, The Astronomical Journal, 133, 2222, doi: 10.1086/513517

Shen, Y., Richards, G. T., Strauss, M. A., et al. 2011, The Astrophysical Journal Supplement Series, 194, 45, doi: 10.1088/0067-0049/194/2/45

Sijacki, D., Vogelsberger, M., Genel, S., et al. 2015, Monthly Notices of the Royal Astronomical Society, 452, 575, doi: 10.1093/mnras/stv1340

Silk, J., \& Rees, M. J. 1998, Astronomy and Astrophysics, 331, 4

Silverman, J. D., Kampczyk, P., Jahnke, K., et al. 2011, The Astrophysical Journal, 743, 2, doi: 10.1088/0004-637X/743/1/2

Smethurst, R. J., Simmons, B. D., Lintott, C. J., \& Shanahan, J. 2019, Monthly Notices of the Royal Astronomical Society, 489, 4016, doi: $10.1093 / \mathrm{mnras} / \mathrm{stz} 2443$

Snyder, G. F., Rodriguez-Gomez, V., Lotz, J. M., et al. 2019, Monthly Notices of the Royal Astronomical Society, 486, 3702, doi: 10.1093/mnras/stz1059

Somerville, R. S., Hopkins, P. F., Cox, T. J., Robertson, B. E., \& Hernquist, L. 2008, Monthly Notices of the Royal Astronomical Society, 391, 481, doi: 10.1111/j.1365-2966.2008.13805.x

Springel, V., Di Matteo, T., \& Hernquist, L. 2005, \mnras, 361, 776, doi: 10.1111/j.1365-2966.2005.09238.x

Steinborn, L. K., Hirschmann, M., Dolag, K., et al. 2018, Monthly Notices of the Royal Astronomical Society, 481, 341, doi: 10.1093/mnras/sty2288

Terrazas, B. A., Bell, E. F., Pillepich, A., et al. 2019, arXiv e-prints, 1906, arXiv:1906.02747. http://adsabs.harvard.edu/abs/2019arXiv190602747T 
Treister, E., Schawinski, K., Urry, C. M., \& Simmons, B. D. 2012, The Astrophysical Journal, 758, L39, doi: 10.1088/2041-8205/758/2/L39

Truong, N., Pillepich, A., Werner, N., et al. 2019, arXiv:1911.11165 [astro-ph]. http://arxiv.org/abs/1911.11165

Urbano-Mayorgas, J. J., Villar Martn, M., Buitrago, F., et al. 2019, Monthly Notices of the Royal Astronomical Society, 483, 1829, doi: 10.1093/mnras/sty2910

Urrutia, T., Lacy, M., \& Becker, R. H. 2008, The Astrophysical Journal, 674, 80, doi: 10.1086/523959

Valentini, M., Murante, G., Borgani, S., et al. 2019, arXiv:1911.02572 [astro-ph]. http://arxiv.org/abs/1911.02572

van der Wel, A., Franx, M., van Dokkum, P. G., et al. 2014, The Astrophysical Journal, 788, 28, doi: 10.1088/0004-637X/788/1/28

Ventou, E., Contini, T., Bouch, N., et al. 2017, Astronomy \& Astrophysics, 608, A9, doi: 10.1051/0004-6361/201731586

—. 2019, arXiv e-prints, 1909, arXiv:1909.03706. http://adsabs.harvard.edu/abs/2019arXiv190903706V

Vestergaard, M., \& Peterson, B. M. 2006, The Astrophysical Journal, 641, 689, doi: 10.1086/500572
Villforth, C., Herbst, H., Hamann, F., et al. 2019, Monthly Notices of the Royal Astronomical Society, 483, 2441, doi: 10.1093/mnras/sty3271

Villforth, C., Hamann, F., Rosario, D. J., et al. 2014, Monthly Notices of the Royal Astronomical Society, 439, 3342, doi: 10.1093/mnras/stu173

Villforth, C., Hamilton, T., Pawlik, M. M., et al. 2017, Monthly Notices of the Royal Astronomical Society, 466, 812, doi: 10.1093/mnras/stw3037

Weigel, A. K., Schawinski, K., Treister, E., Trakhtenbrot, B., \& Sanders, D. B. 2018, Monthly Notices of the Royal Astronomical Society, 476, 2308, doi: $10.1093 / \mathrm{mnras} / \mathrm{sty} 383$

Weinberger, R., Springel, V., Pakmor, R., et al. 2018, Monthly Notices of the Royal Astronomical Society, 479, 4056, doi: 10.1093/mnras/sty1733

Wild, V., Heckman, T., \& Charlot, S. 2010, Monthly Notices of the Royal Astronomical Society, doi: 10.1111/j.1365-2966.2010.16536.x

Xu, C. K., Zhao, Y., Scoville, N., et al. 2012, The Astrophysical Journal, 747, 85, doi: 10.1088/0004-637X/747/2/85

Yue, M., Fan, X., Schindler, J.-T., McGreer, I. D., \& Huang, Y.-H. 2019, The Astrophysical Journal, 883, 141, doi: 10.3847/1538-4357/ab3db2

Zhao, D., Ho, L. C., Zhao, Y., Shangguan, J., \& Kim, M. 2019, The Astrophysical Journal, 877, 52, doi: $10.3847 / 1538-4357 /$ ab1921 